\title{
Structural Rearrangements of MscS during Activation Gating
}

\author{
Valeria Vásquez \\ Maracaibo, Venezuela
}

Licentiate, Universidad Central de Venezuela, 1999

A Dissertation presented to the Graduate Faculty of the University of Virginia in Candidacy for the Degree of Doctor of Philosophy

Department of Molecular Physiology and Biological Physics

University of Virginia

May, 2008 


\section{TABLE OF CONTENTS}

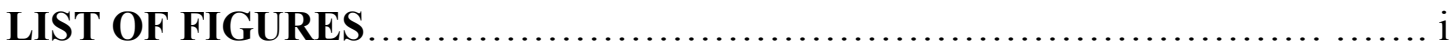

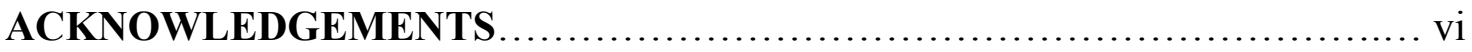

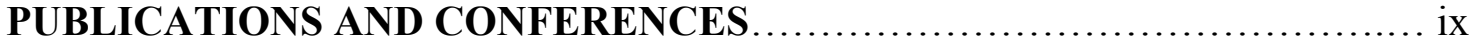

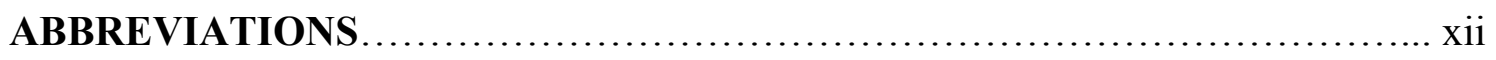

\section{CHAPTER I. Introduction}

I.1 Abstract.............................................................. 1

I.2 Introduction

I.2.1 Mechanosensitive Channels....................................... 3

a) Prokaryotic Mechanosensitive Channels............................ 5

b) Mechanosensitive Channel of Small Conductance $(M s c S) \ldots \ldots \ldots \ldots \ldots . . . .11$

c) Lipid-Protein Interactions and Channel Activation................... 12

I.2.2 Site-Directed Spin Labeling and Electron Paramagnetic Resonance

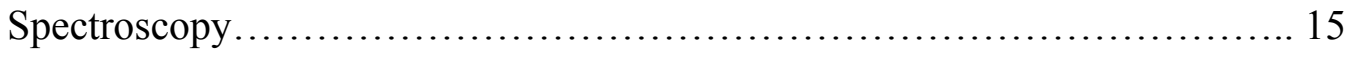

I.3 Thesis Outline...................................................... 20

CHAPTER II: An Optimized Purification and Reconstitution Method for

MscS Channel: Strategies for Spectroscopical Analysis

II.1 Abstract................................................................ 22

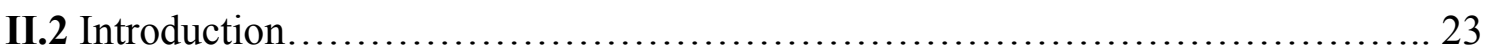

II.3 Experimental Procedures

II.3.1 Materials...................................................... 26

II.3.2 Constructs and Strains....................................... 26

II.3.3 Functional Assay................................................. 27 
II.3.4 Expression and Purification of MscS in E. coli cells.................. 27

II.3.5 Molecular Mass Determination by Static Light Scattering............... 28

II.3.6 MscS Reconstitution........................................ 29

II.3.7 Liposome Patch-Clamp....................................... 29

II.3.8 EPR Spectroscopy and Analysis................................ 30

II.3.9 Fluorescence Spectroscopy.................................. 30

II.4 Results

II.4.1 Functional Characterization of His-tagged MscS constructs........... 31

II.4.2 Oligomeric Behavior of MscS under various Purification Conditions.... 31

II.4.3 Functional Characterization of Purified Channel...................... 37

II.4.4 Two-Dimensional Aggregation of Reconstituted MscS............... 37

II.5 Discussion...................................................... 43

CHAPTER III: Three Dimensional Architecture of Membrane-Embedded MscS in the Closed Conformation

III.1 Abstract........................................................ 47

III.2 Introduction...................................................... 48

III.3 Experimental Procedures

III.3.1 Mutagenesis, Expression, Spin-Labeling, and Reconstitution

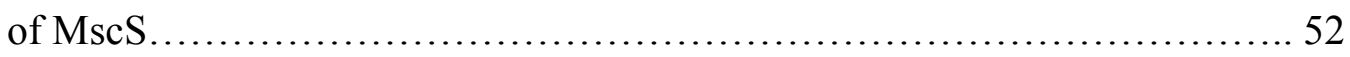

III.3.2 Pegylation............................................... 52

III.3.3 Liposome and Spheroplast Patch Clamp......................... 52

III.3.4 EPR Spectroscopy and Data Analysis.......................... 53

III.3.5 Functional Assays......................................... 54 
III.3.6 MscS N-terminal Model and Pseudo-Atom driven Solvent Accessibility Refinement............................................ 54

III.3.7 Molecular Dynamics Simulations............................... 55

III.4 Results

III.4.1 Expression and Purification of MscS Cysteine Mutants.............. 57

III.4.2 Environmental Parameters of MscS TM segments.................. 60

III.4.3 Conformation of the N-terminal segment......................... 69

III.4.4 Three-Dimensional Model for the N-terminus and TM segments

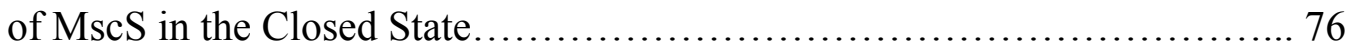

III.5 Discussion......................................................... 83

CHAPTER IV: Three Dimensional Architecture of Membrane-Embedded MscS in the Open Conformation. Conformational Rearrangements in MscS

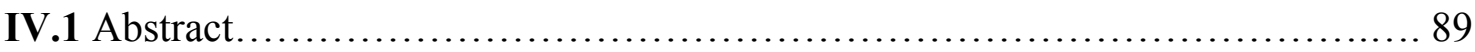

IV.2 Introduction............................................................ 90

IV.3 Experimental Procedures

IV.3.1 Spheroplast Patch-Clamp.................................. 93

IV.3.2 Mutagenesis, expression, spin labeling, and reconstitution of MscS... 93

IV.3.3 EPR spectroscopy and data analysis.............................. 94

IV.4 Results

IV.4.1 Stabilization of $\mathrm{MscS}$ open state by asymmetric incorporation of

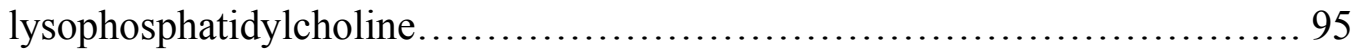

IV.4.2 Structural Rearrangement of MscS Transmembrane Domain upon

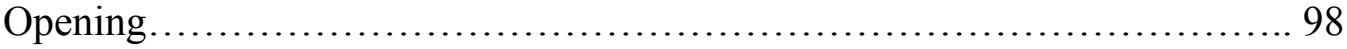


IV.4.3 Analysis of the Conformational Rearrangements during Activation

Gating. 106

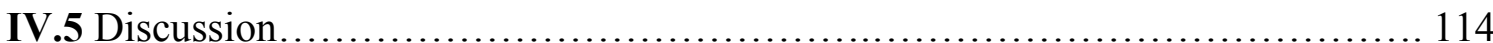

\section{CHAPTER V: Understanding the Molecular Basis of the Voltage-Modulation}

\section{in MscS}

V.1 Abstract

V.2 Introduction

V.3 Experimental Procedures

V.3.1 Constructs, Strains, and Mutagenesis.

V.3.2 Spheroplast Patch-Clamp..................................... 129

V.3.3 Molecular Dynamics Simulations.................................. 131

V.3.4 Functional Assays............................................. 132

V.3.5 Expression Purification, and Gel Filtration Analysis................... 132

V.3.6 MscS Reconstitution........................................ 132

V.3.7 EPR Spectroscopy and Data Analysis.......................... 133

V.4 Results and Discussion

V.4.1 MscS is a slightly Anion-selective, Pressure Activated Ion Channel..... 134

V.4.2 MscS Crystal Structure Exhibits Low Conductance and High Anionic

Selectivity.

V.4.3 Locating the Charges Responsible for the Voltage Dependence of the Inactivation in $\mathrm{MscS}$

CHAPTER VI: Conclusions and Future Perspectives....................... 151

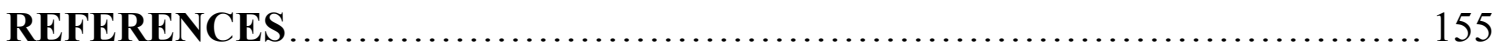




\section{LIST OF FIGURES}

\section{CHAPTER I}

I.1 Mechanosensitive channel super-family.............................. 4

I.2 Role of mechanosensitive channel in Gram-negative bacteria................. 6

I.3 Mechanosensitive channels in E. coli plasma membrane..................... 7

I.4 Comparison of MscL and MscS structures........................... 10

I.5 Changes in the force profile of the lipid bilayer are the trigger for

Mechanosensitive channels opening..................................... 14

I.6 Structural parameters derived from EPR experiments....................... 17

CHAPTER II: An Optimized Purification and Reconstitution Method of MscS

\section{Channel: Strategies for Spectroscopical Analysis}

II.1 Functional characterization of different MscS constructs................... 25

II.2 Evaluation of different MscS constructs and purification conditions............ 35

II.3 Molecular mass determination of purified MscS through ASTRA software analysis (Wyatt Technology Corp.) of SEC-LS/UV/RI data..................... 36

II.4 Single-channel records from purified MscS in asymmetric solutions ........... 39

II.5 MscS mobility profiles monitored by $\mathrm{CW}$-EPR from spin-labels attached to Cys-residues in the TM1 .............................................. 40

II.6 MscS two-dimensional aggregation in liposomes measured by fluorescence resonance energy transfer (FRET), using fluorescein or tetramethyl-rhodamine as the donor/acceptor pair................................................ 42 


\section{CHAPTER III: Three Dimensional Architecture of Membrane-Embedded}

\section{MscS in the Closed Conformation}

III.1 MscS architecture and expression of cysteine mutants.................... 51

III.2 Biochemical evaluation of MscS cysteine mutants...................... 59

III.3 MscS closed state characterization.................................. 63

III.4 Labeling efficiency as determined by cysteine pegylation................... 64

III.5 EPR spectroscopy of MscS...................................... 65

III.6 Vector analysis for oxygen accessibility data........................... 66

III.7 Identification of MscS interfacial residues with DOGS-NTA[Ni(II)]lipids..... 68

III.8 Functional and residue-specific environmental parameter profiles of

$\mathrm{NH}_{2}$-terminal cysteine mutants......................................... 70

III.9 EPR spectroscopy of MscS with an N-terminal His-tag.................... 73

III.10 Fourier transform power spectrum of the INiEdda profile................. 74

III.11 Structural Analysis of the $\mathrm{NH}_{2}$-terminal segment....................... 75

III.12 Topology and structural model of the N-terminal domain of MscS, obtained

by the pseudo-atom driven solvent accessibility refinement.................... 77

III.13 Environmental phase diagram of the N-terminus and TM segments based on

mobility, $\mathrm{O}_{2}$ accessibility, and $\mathrm{NiEdda}$ accessibility parameters.................. 78

III.14 MscS closed conformation obtained from EPR based refinement ........... 81

III.15 All-atom MD simulations of EPR based MscS model and MscS

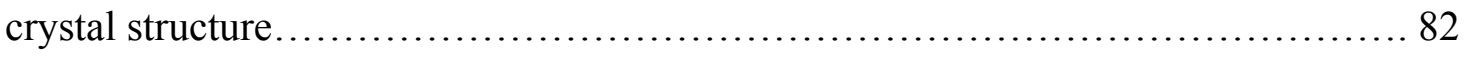

III.16 Comparison of MscS crystal structure and the outcome of EPR

based refinement...................................................... 86 
CHAPTER IV: Three Dimensional Architecture of Membrane-Embedded MscS in the Open Conformation.

IV.1 MscS does not inactivate upon Lyso PC activation 97

IV.2 Asymmetric incorporation of Lyso PC induces conformational changes in

$\mathrm{MscS}$

IV.3 EPR spectroscopy of MscS upon addition of Lyso PC

IV.4 Extent of environmental parameter changes of MscS, upon addition of

Lyso PC

IV.5 Residue environmental parameter profiles mapped onto molecular surfaces, of two representative monomers, rendering the closed (from EPR refinement) and a non-conductive conformations (crystal structure) 104

IV.6 Extent of environmental parameter changes, upon MscS opening, mapped onto molecular surfaces of the closed (EPR-based model) and a non-conductive conformations (crystal structure).

IV.7 Calculation of the Fourier transform power spectrum of the accessibility profiles for the transmembrane domain in the closed and open conformations.... 108

IV.8 Window periodicity analysis for MscS transmembrane domain in the closed and open states

IV.9 Structural analysis of MscS transmembrane domain in the open conformation... 112

IV.10 Schematic representation of the structural rearrangements underlying gating in $\mathrm{MscS}$.

IV.11 Functional differences between MscS and MscL 116 


\section{CHAPTER V: Understanding the Molecular Basis of the Voltage-Modulation in}

\section{MscS}

V.1 Architecture of MscS and Sequence Alignment of its Transmembrane

Domain.

V.2 Current-voltage relationship of wild-type MscS

V.3 MscS activation gating is dependent on tension and modulated by voltage.

V.4 Ionic currents through $\mathrm{MscS}$ as determined by all-atom MD simulations.

V.5 Neutralization of charged residues in the TM1-TM2 hairpin.

V.6. Salt-bridge formation as determined through all-atom MD simulations of

MscS and functional assays.

V.7. Hydrodynamic properties of MscS and EPR spectroscopy of MscS salt bridge

mutants. 
"I am among those who think that science has great beauty. A scientist in his laboratory is not only a technician: he is also a child placed before natural phenomena which impress him like a fairy tale" 


\section{ACKNOWLEDGMENTS}

I would like to express my sincere gratitude to my advisor Eduardo Perozo. He has been a constant source of support and guidance during my graduate studies. More than an advisor, Eduardo has been a good friend, always honest and willing to hear new ideas and approaches. I feel that it has been a great privilege to work with Eduardo, personally and scientifically.

I sincerely want to acknowledge current and past members of Perozo's Lab for always giving me ideas and suggestions during my thesis. In particular, I would like to thank D. Marien Cortes, for her kind support during this project and her very good advices in molecular biology and biochemistry. I thank Luis Cuello for teaching me how to gently treat membrane proteins, and moreover for his experimental advice throughout my thesis. I also want to thank Christopher Ptak for teaching me how to use a CW-EPR instrument and for his generous help at the beginning of my research. I wish to thank Sudha Chakrapani, Vishwanath Jogini, and H. Raghuraman for teaching me channel kinetics, molecular dynamics, and fluorescence, respectively; moreover for offering me their great friendship and constant support, and for trying to improve my speaking and writing skills. I would like to also acknowledge the professional and friendly atmosphere provided in the lab by: Yi-Shiuan Liu, Thep Sompornpisut, Toi Sompornpisut, Jie Wu, Carlos Gonzalez, Olivier Dalmas, and Jose Santos.

I would always be grateful to Boris Martinac, for teaching me how to patch spheroplasts and liposomes in a hilarious manner, and more than that for its contagious love for biophysics and mechanosensitive channels. I sincerely thank Marcos Sotomayor, for his constant help and support in this difficult journey called MscS. Moreover, he 
introduced me to what I used to consider a black box, "MD simulations". I hope we can work together in the future.

My thanks also go to the members of my committee, Dr. Doug Bayliss, Dr. David Cafiso, Dr. Jeff Holt, and Dr. Lukas Tamm, for providing many valuable comments that improved the presentation and contents of my thesis. I gratefully acknowledge Dr. Robert Nakamoto for his endless support and encouragement throughout my PhD studies. Dr. Nakamoto was incredibly helpful and thoughtful in the transition from UVA to U of C, without him it would have never been as smooth as it was.

I am very grateful to Pam Mullinex, Carol Yowell, Cathy Black (at UVA) and Laura Wright (at $\mathrm{U}$ of $\mathrm{C}$ ) for making all the paperwork a piece of cake and for their invariable good mood.

I would always be deeply indebted to my Family: Enrique, Lulu, Mariaga, Juan Francisco, Daniela, Juan Andres, Carla, Arthur, Carlos Eduardo, Eduardo, Ana, Anaeva, and Ana Maria. I would always be grateful to them for always being there, for teaching the most important lessons in my life, for their incredible and endless support no matter what I decided to do, and for simply being the joy of my life. I also want to thank my inlaw family (the Cordero-Morales and the Cordero-Mastrodomenico) for making me feel welcome and for their constant encouragement.

At last but not least, I would really want to dedicate my special thanks to the person I consider it has help me the most. Julio besides being a great husband and a terrific scientist, he has also been a diligent classmate, an ideal study-pal, an always willing to help lab-colleague, and more importantly he has been a perfect companion in making my life an easy ride. The solitude I could have feel for being away from my 
family and my country never resided more than one minute thanks to Julio's happiness and willingness to make me feel better. For infinite reasons, I dedicate this thesis to Julio. 


\section{PUBLICATIONS AND CONFERENCES}

\section{Publications:}

1- Serdar Uysal, Valeria Vásquez, Valentina Terechko, Frederic A. Fellouse, Kaori Esaki, Sachdev S. Sidhu, Shohei Koide, Anthony A. Kossiakoff, and Eduardo Perozo. 2008. Crystal Structure of Full-length KcsA at 3.7 A. In preparation.

2- Valeria Vásquez, Marcos Sotomayor, Julio Cordero-Morales, Klaus Schulten, and Eduardo Perozo. 2008. Structural Rearrangements of MscS during Activation Gating.

\section{Submitted.}

3- Valeria Vásquez, Marcos Sotomayor, D. Marien Cortes, Benoît Roux, Klaus Schulten, and Eduardo Perozo. 2008. Three Dimensional Architecture of MembraneEmbedded MscS in the Closed Conformation. Journal of Molecular Biology 378(1): 5570.

4- Julio Cordero-Morales, Vishwanath Jogini, Anthony Lewis, Valeria Vásquez, D. Marien Cortes, Benoît Roux, and Eduardo Perozo. 2007. Molecular Driving Forces Determining Potassium Channel Slow Inactivation. Nature Structural \& Molecular Biology 14(11): 1062-1069.

5- Valeria Vásquez, D. Marien Cortes, Hiro Furukawa, and Eduardo Perozo. 2007. An Optimized Purification and Reconstitution Method for the MscS Channel: Strategies for Spectroscopical Analysis. Biochemistry 46(23): 6766-6773.

6- Marcos Sotomayor*, Valeria Vásquez*, Eduardo Perozo, and Klaus Schulten. 2007. Ion Conduction through $\mathrm{MscS}$ as Determined by Electrophysiology and Simulation. Biophysical Journal 92(3): 886-902. *M. Sotomayor and V. Vasquez contributed equally to this work. 
Conferences:

1- Vásquez, V; Cordero-Morales, JF; Cortes, DM; Perozo, E. 2007. Structural Dynamics of MscS Transmembrane Segments in the Open State. A Site-directed Spin-labeling Analysis (Platform). 52 ${ }^{\text {nd }}$ Annual meeting of the Biophysical Society, Long Beach, CA. 2- Uysal, S; Vásquez, V; Terechko, V; Fellouse, FA; Esaki, A; Sidhu, SS; Koide, S; Kossiakoff, A; Perozo, E. 2007. Crystal Structure of Full-length KcsA at $3.7 \AA$ (Platform). 52 ${ }^{\text {nd }}$ Annual meeting of the Biophysical Society, Long Beach, CA.

3- Vásquez, V; Sotomayor, M; Cortes, DM; Roux, B; Schulten, K; Perozo, E. 2007. Three Dimensional Architecture of Membrane-Embedded MscS in the Closed Conformation (Poster). Gordon Research Conference on "Mechanosensory Transduction", University of New England, Biddeford, Maine.

4- Vásquez, V; Sotomayor, M; Cortes, DM; Roux, B; Schulten, K; Perozo, E. 2007. Three Dimensional Architecture of MscS N-terminus and TM Segments in the Closed State. (Poster). $51^{\text {st }}$ Annual meeting of the Biophysical Society, Baltimore, MD.

5- Vásquez, V; Cortes, DM; Furukawa, H; Perozo, E. 2007. An Optimized Purification and Reconstitution Method for MscS. (Poster). $51^{\text {st }}$ Annual meeting of the Biophysical Society, Baltimore, MD.

6- Sotomayor, M; Vásquez, V; Perozo, E; Schulten, K. 2007. Ion Conduction through $\mathrm{MscS}$ as Determined by Electrophysiology and Simulation. (Poster). $51^{\text {st }}$ Annual meeting of the Biophysical Society, Baltimore, MD.

7- Cordero-Morales, JF; Cortes, DM; Chakrapani, S; Jogini, V; Cuello, LG; Vásquez, V; Perozo, E. 2007. Multiple Gating Modes in KcsA are Determined by the Selectivity Filter Dynamics. (Platform). 51 ${ }^{\text {st }}$ Annual meeting of the Biophysical Society, Baltimore, MD. 
8- Vásquez, V. 2005. Structural Transitions in Mechano-Gated Channels. (Symposium, invited speaker). XXXV International Congress of Physiological Sciences, San Diego, CA.

9- Vásquez, V; Cortes, DM; Perozo, E. 2005. Structural Dynamics of MscS Transmembrane Segments in the Closed State. A Site-directed Spin-labeling Analysis. (Poster). $49^{\text {th }}$ Annual meeting of the Biophysical Society, Long Beach, CA.

10- Vásquez, V; Perozo, E. 2004. Voltage Dependent Gating in MscS. (Poster). $48^{\text {th }}$ Annual meeting of the Biophysical Society, Baltimore, MD. 


\section{ABBREVIATIONS}

CW-EPR: Continuous wave electron paramagnetic resonance

DDM: n-Dodecyl- $\beta$-D-Maltopyranoside

DOPC: 1,2-Dioleoyl-sn-Glycero-3-Phosphocholine

IPTG: Isopropyl- $\beta$-D-thiogalactopyranoside

Lyso PC: Lysophosphatidylcholine

PBS: Phosphate-buffer saline

PMSF: Phenylmethylsulfonyl fluoride

POPE: 1-Palmitoyl-2-Oleoyl-sn-Glycero-3-Phosphoethanolamine

POPG: 1-Palmitoyl-2-Oleoyl-sn-Glycero-3-[Phospho-rac-(1-glycerol)]

Spin label: (1-Oxyl-2,2,5,5-tetramethylpyrrolidin-3-yl) Methyl Methanethiosulfonate

TCEP: Tris-(2-Carboxyethyl)-Phosphine Hydrochloride

TCYP: Tris-(2-Cyanoethyl)-Phosphine

TM: Transmembrane 


\section{CHAPTER I. Introduction}

\section{I.1 ABSTRACT}

The mechanosensitive channel of small conductance $(\mathrm{MscS})$ is part of a coordinated response to osmotic challenges in E. coli. MscS is particularly interesting since it is activated by changes in membrane tension and its inactivation is modulated by transmembrane voltage. Its crystal structure depicts a homoheptamer, in which each of the subunits has three TM segments and a large cytoplasmic domain. At first glance, the crystal structure seemed to be trapped in an open state based on the calculated diameter of the permeation pathway that appeared to agree with MscS single channel conductance. In this work using electrophysiological experiments and MD simulations we were able to determine that the crystal represents a non-conductive conformation. The crystal structure also suggested that several arginines were exposed to the lipids in TM1 and TM2, thus, it was proposed that these charged residues could act as voltage sensors. We have experimentally tested this proposal and we have found that none of the TM charges are involved in the $\mathrm{MscS}$ voltage sensing process. On the other hand, we established that an inter-subunit salt-bridge connecting the TM domain with the cytoplasmic basket influences the inactivation process, but the voltage sensor entity still remains ambiguous. In an effort to understand the molecular basis of $\mathrm{MscS}$ gating mechanism, we have improved the strategies for MscS spectroscopical analysis by optimizing the available purification and reconstitution protocols. We also have combined different approaches such as spin-labeling-EPR, high throughput functional assays and patch-clamp methods with classical molecular biology and biochemical procedures. By manipulating the location of reactive cysteines, we have introduced probes along MscS transmembrane 
domain. With these reporters, we obtained information on the topology, secondary, and tertiary structure of the closed and open conformations, using EPR analysis of spin labeled mutants under native-like conditions. In the closed conformation, MscS shows a more compact TM domain than in the crystal structure, described by a realignment of the TM segments towards the normal of the membrane, with the previously unresolved $\mathrm{NH}_{2}$ terminus placed at the periplasmic interface of the lipid bilayer. Whereas, the transition to the open state is characterized by an increase in overall dynamics, that involves major rearrangements such as burying of the $\mathrm{NH}_{2}$-terminus in the membrane, tilting of TM1TM2 segments, expansion, rotation, and wetting of the TM3 helices. The present threedimensional models of membrane-embedded $\mathrm{MscS}$ in the closed state and the open conformation (in progress) represent key steps in determining the molecular mechanism of MscS gating. 


\section{I.2 INTRODUCTION}

\section{I.2.1 Mechanosensitive Channels}

Mechanotransduction plays an important role in a wide range of physiological processes such as touch, pain, hearing, balance, proprioception, blood pressure regulation, turgor control in plant cells, and regulation of cell volume. The molecular basis for mechanosensation requires the understanding of the energy transduction that converts the stimuli into cell response; in most of the systems this conversion occurs through mechanosensitive ion channels. It has been suggested that they might have evolved as cellular osmoregulators that sensed small changes in the concentration of water across membranes of primitive cells such as bacteria (Batiza et al., 1999; Blount, 2003; Hamill and Martinac, 2001; Kung, 2005; Martinac, 2001, 2004; Perozo, 2006). Mechanosensitive channels belong to a heterogeneous group, since they are oligomeric membrane proteins that have been classified according to their function rather than topology. Thus, by definition, mechanosensitive channels are those whose probability of opening is altered by mechanical stresses. This super-family includes (Figure I.1) members of the two-pore domain potassium channels (Maingret et al., 1999a; Patel et al., 1998), the amiloride-sensitive sodium channels (Kellenberger and Schild, 2002), the TRP channels (Corey, 2003), and the prokaryotic mechanosensitive channels (Berrier et al., 1996). 
A

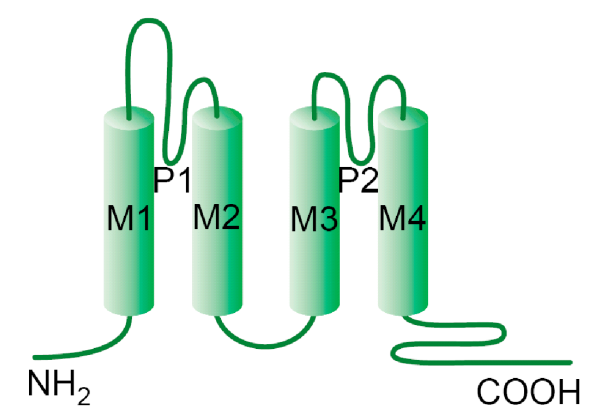

C

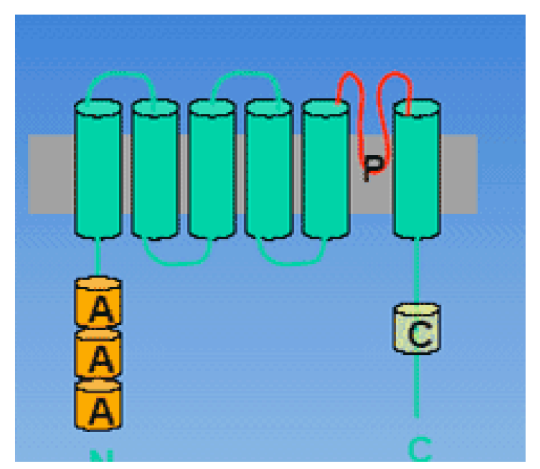

B

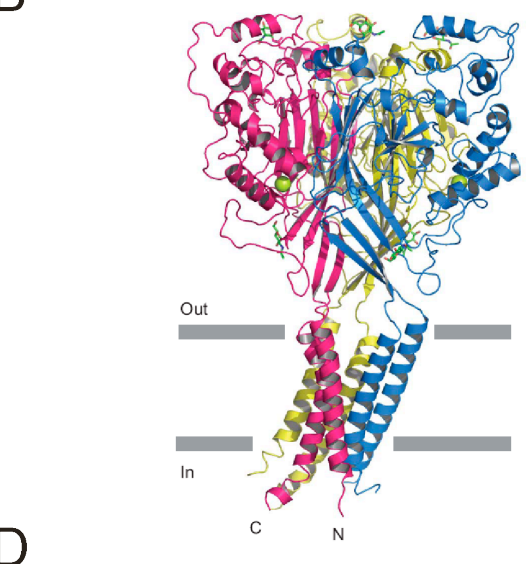

D

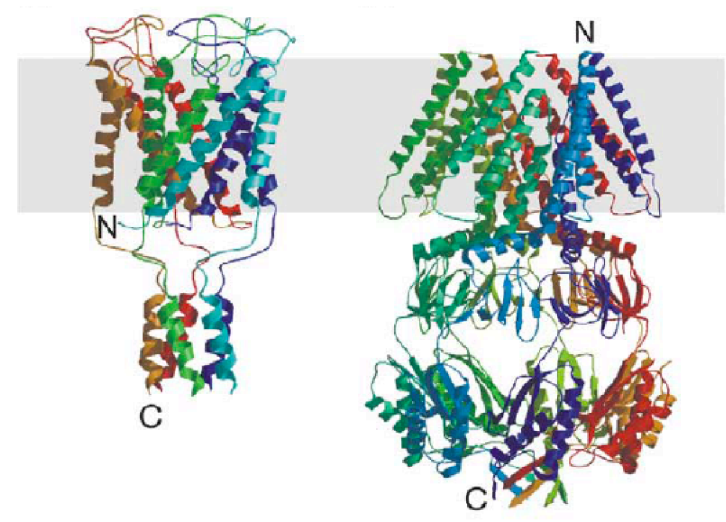

Figure I.1. Mechanosensitive channel super-family. A) Cartoon depicting a two pore domain potassium channel. B) Structure of the chicken acid-sensing ion channel (ASIC1a), recently solved (Jasti et al., 2007), related to the epithelial sodium channel family (ENaCs). C) Cartoon representing a TRP channel with ankirin repeats at the $\mathrm{COOH}$-terminal domain. D) Structure of two prokaryotic mechanosensitive channels. Left panel, the mechanosensitive channel of large conductance MscL (Chang et al., 1998). Right panel, the mechanosensitive channel of small conductance MscS (Bass et al., 2002). 
Based on the way these channels are activated, they could be classified into two classes, one that sense stress from fibrillar proteins and other from the membrane (Markin and Sachs, 2007). The first kind is associated with specialized receptors such as cochlear hair cells (Hackney and Furness, 1995), and touch receptors in C. elegans (GarciaAnoveros and Corey, 1996), and in D. melanogaster (Walker et al., 2000). The second type are channels activated by membrane stress which are universally distributed (Hamill and Martinac, 2001), and do not seem to require an intact intra or extracellular matrix in order to function (Delcour et al., 1989). This family of ion channels is worth to be studied since abnormalities in their function can cause neuronal and muscular degeneration, cardiac arrhythmias, hypertension, and polycystic kidney disorder, among other diseases. Despite their obvious biological relevance, the molecular basis of mechanosensory signaling pathways in living cells remains largely unknown (Martinac, 2004).

\section{a) Prokaryotic Mechanosensitive Channels}

The prokaryotic mechanosensitive channels are a key component in the emergency response of prokaryotes to sudden osmotic downshock (Figure I.2). These channels are classified according to their conductance and to the amount of tension that is needed to activate them as MscL, MscS/MscK, and MscM; for mechanosensitive channel of Large, Small, and Mini conductance, respectively (Figure I.3). Particularly, MscL and MscS have been shown to play a critical role in osmoregulation, since knockout cells lyse upon osmotic downshock (Batiza et al., 2002; Levina et al., 1999). 


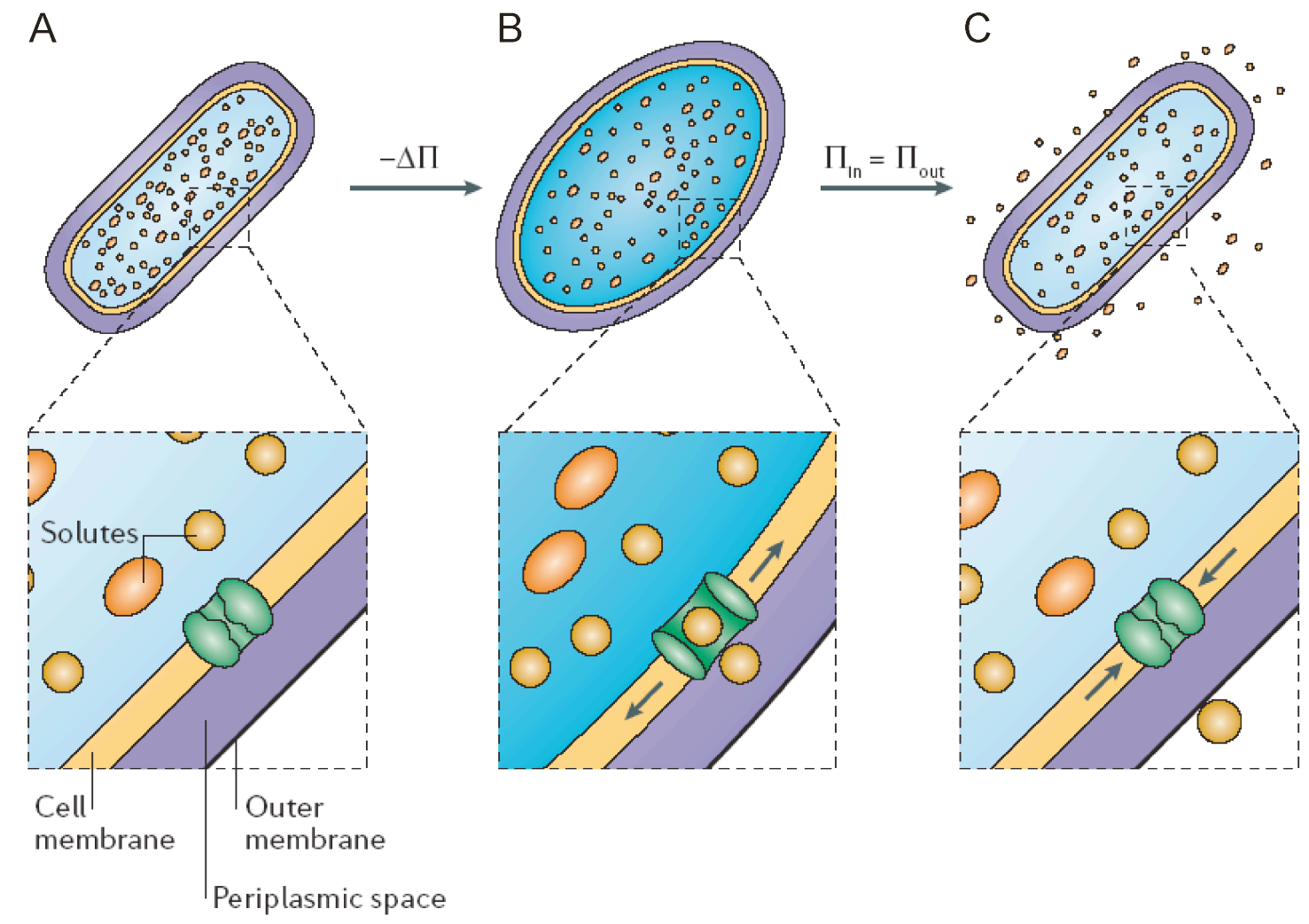

Figure I.2. Role of mechanosensitive channel in Gram-negative bacteria. Mechanosensitive channels function as osmotic emergency valves in E. coli cells, as they open upon hypo-osmotic challenges. A) Mechanosensitive channels are closed when cells are in osmotic equilibrium. B) When the extracellular environment becomes hypo-osmotic, solvent enters the cell inducing changes in the membrane lateral tension, therefore leading to channel opening an a rapid release of solutes, C) Once the turgor pressure decreases, the channels return to the closed conformation. (Perozo, 2006). 


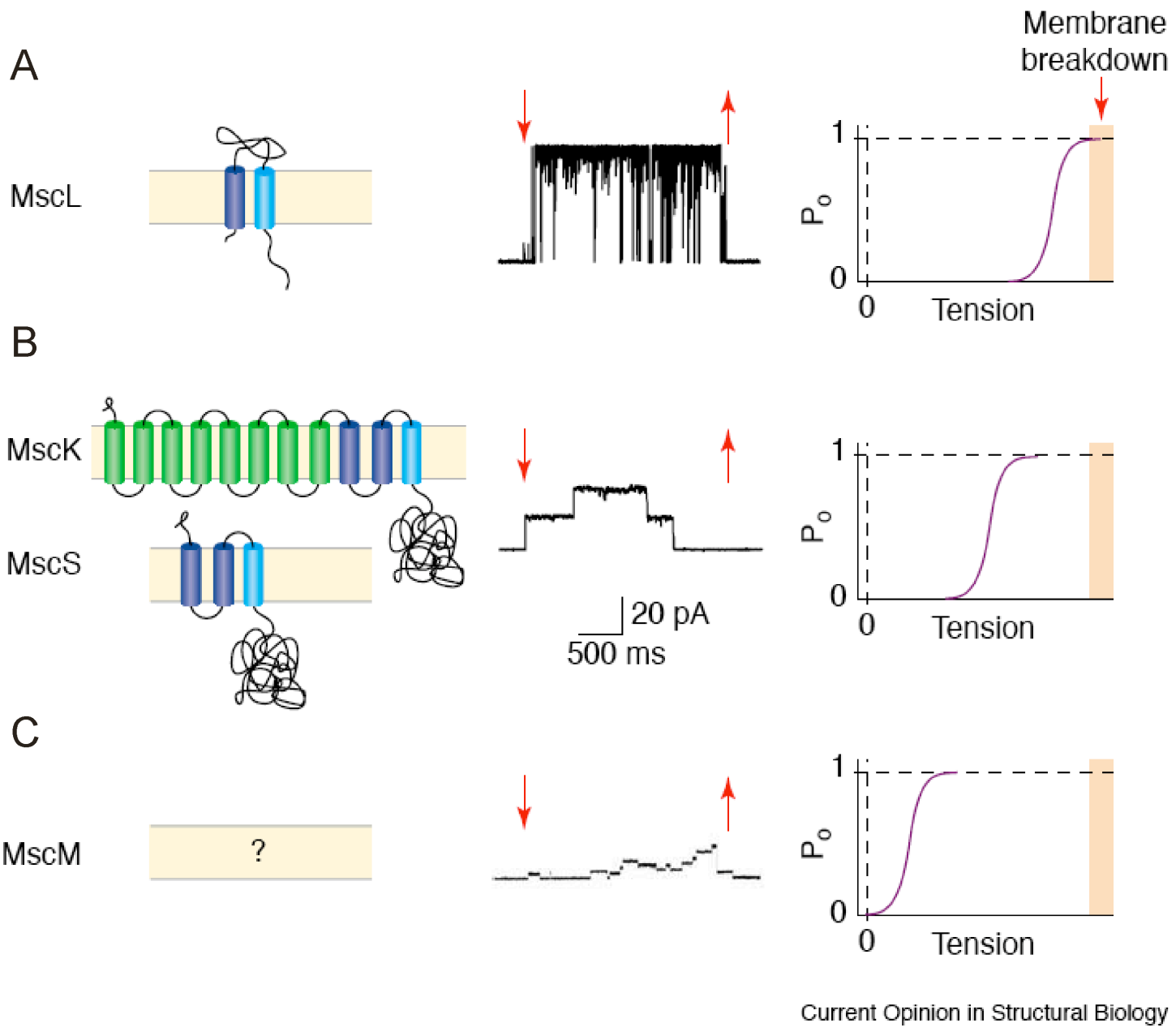

Figure I.3. Mechanosensitive channels in E. coli plasma membrane. Left panel illustrates channel topology, middle panel single channel activity, and right panel activation threshold. A) MscL is a homoheptamer in which each monomer has two TM segments. Its single channel conductance is $\sim 3.5 \mathrm{nS}$, and its activation tension is approximately 10 dyne $/ \mathrm{cm}$. B) MscS, as originally described, is actually the result of two gene products ( $\mathrm{MscK}$ and $\mathrm{MscS}$ ). MscS is a homoheptamer in which each monomer has three TM segments and a cytoplasmic basket. MscK structure is still unknown. MscK stands for its preference for potassium over other ions. Both channels have similar single channel conductance $(\sim 1 \mathrm{nS})$ and are activated at 5 dyne $/ \mathrm{cm} . \mathrm{C})$ MscM activity is characterized by single channel conductance in the 100-200 pS range. So far its molecular identity has not been determined (Perozo and Rees, 2003) 
When MscS was first characterized, its activity appeared to be more complicated than MscL (Buechner et al., 1990). This complexity partially came from the fact that two similar, but distinguishable, activities at the single channel level were measured. Later on, two independent homologous genes were cloned. The first one $\operatorname{YggB}$ codes for MscS, and the second one KefA corresponds to MscK (Levina et al., 1999). MscS and MscK are 286 and 1120 amino acid residues, respectively; where only 300 residues of the COOHterminal (the pore-forming unit and the cytoplasmic basket) of MscK exhibit sequence similarity to MscS (Figure I.3). Unlike MscS and MscL, MscK activity was only observed when $\mathrm{K}^{+}$was the primary permeant ion, and only in $30 \%$ of the traces in which MscL was also recorded. Although MscK has the same conductance as MscS, there are several differences between these two channels. MscK requires slightly lower tension to activate, has more frequent rapid partial closures, and does not feature a fast current decay process like MscS. Moreover, MscK has not been systematically characterized yet, mostly due to the low probability of finding it in E. coli membranes. The other channel that belongs to this prokaryotic family, MscM, has been even less characterized as its molecular identity has not been found yet and the presence of its activity seems to be preparation dependent.

On the other hand, a considerable amount of information has been gathered on MscL and MscS over the past few years. Both channels have been electro-physiologically characterized (Akitake et al., 2005; Berrier et al., 1996; Koprowski and Kubalski, 1998; Sukharev, 2002; Sukharev et al., 1993), and their crystal structures has been determined (Figure I.4) (Bass et al., 2002; Chang et al., 1998; Steinbacher et al., 2007); yet the gating mechanism is not fully understood, which is required to define common elements in 
mechanosensation. Nevertheless, the determination of the crystal structure of MscL at $3.5 \AA$ resolution provided a framework that has been used to interpret an extensive amount of mutagenic and functional information. MscL is formed by the association of five subunits each having two transmembrane segments (left panel of Figure I.4), and its structure was clearly trapped in the closed state as it was determined experimentally and by computational analysis (Gullingsrud et al., 2001; Perozo et al., 2001). There are several advantages in using MscL as a model to understand the lipid-protein interactions such as: 1) highly stable in a wide variety of detergents, 2$)$ a large conductance $(\sim 3.5 \mathrm{nS})$ that makes it easy to measure by patch-clamp, 3) very weak voltage dependence and rectification, and 4) the absence of an adaptation/inactivation process (for more information on MscL gating process see section I.2.1.c). Even though MscL is a fantastic tool to study the mechanical properties of the lipid bilayer in determining the function of the proteins, the sequenced members of the MscL family are currently restricted to one archaeon, a single fungus, and bacteria, while the MscS family is much more widely distributed in the three domains of life (Pivetti et al., 2003). 


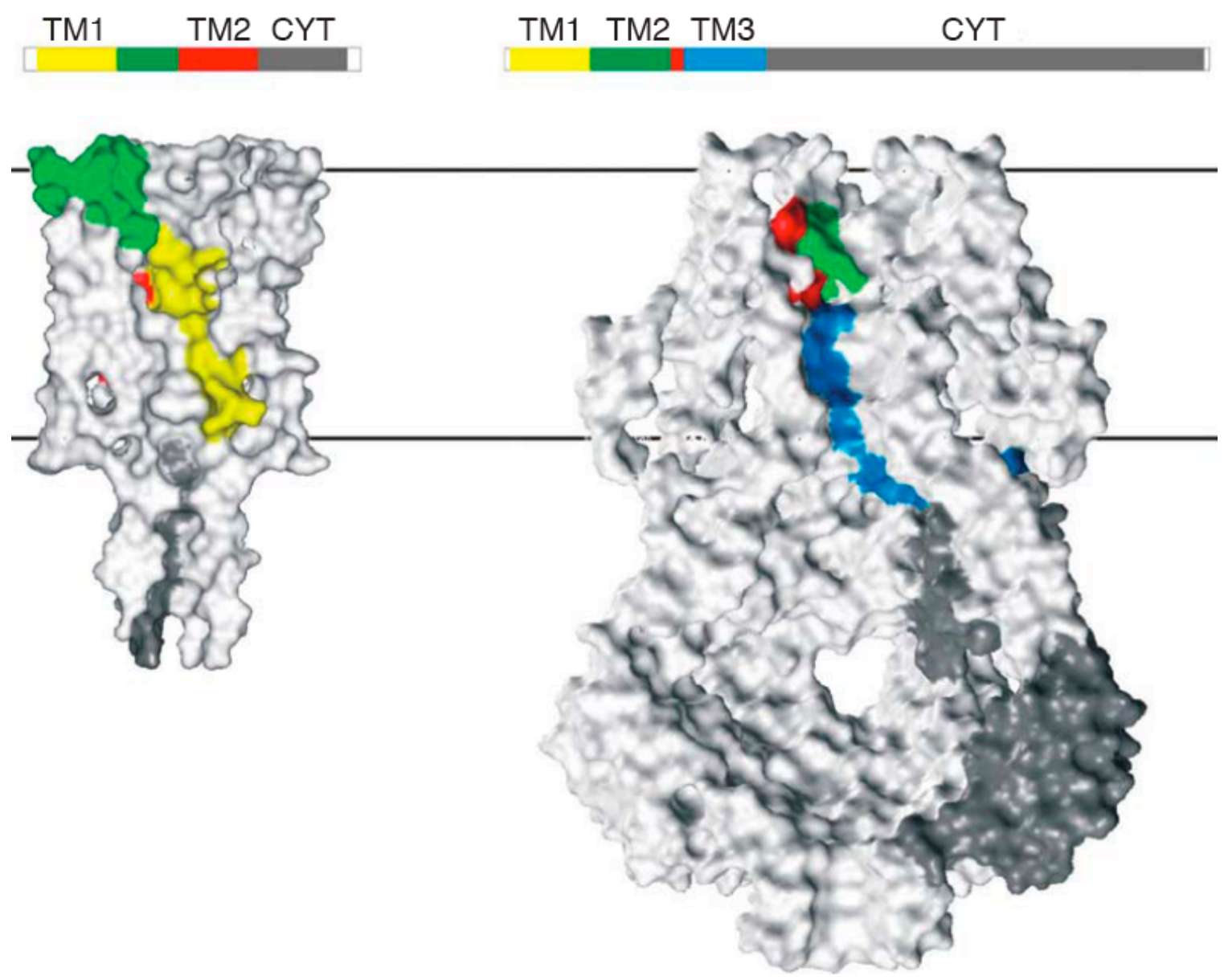

Figure I.4. Comparison of MscL and MscS structures. The channels have been sliced along their centerline in order o show the permeation pathway. Left panel, mechanosensitive channel of large conductance, MscL (Chang et al., 1998). Right panel, mechanosensitive channels of small conductance MscS (Bass et al., 2002). (Bezanilla and Perozo, 2002). 


\section{b) Mechanosensitive Channel of Small Conductance (MscS)}

The activity of MscS, the first to be detected in E. coli cells, was described as a 1 $\mathrm{nS}$ channel conductance that is activated at lower tensions than MscL, and its open probability is also modulated by voltage (Martinac et al., 1987). In contrast to MscL, MscS activity shows a distinct voltage-dependent inactivation process that further complicates its quantitative functional characterization (Akitake et al., 2005). MscS has no significant sequence similarity to MscL, and the crystal structures showed radically different arrangements for both channels (Perozo, 2006). Unlike the voltage sensor, the tension-sensing region of $\mathrm{MscS}$ has been found through in vivo and patch-clamp assays, and it is formed by hydrophobic residues at the lipid-protein interface of the periplasmic and cytosolic side of the membrane (Nomura et al., 2006).

The crystal structure of MscS (Bass et al., 2003; Steinbacher et al., 2007) revealed a homoheptamer with three transmembrane segments and a large cytoplasmic domain with seven openings on the side and one at the bottom (right panel of Figure I.4). The structure appears to be captured in an open state, as suggested by a $7 \AA$ diameter opening at the sevenfold axis of symmetry; however it has to be demonstrated that MscS could open in detergent, in the absence of a membrane. Furthermore, it has been argued using computational analysis that the crystal structure might not represent the open state. Rectification at high voltages, extremely low ionic conductance, and selectivity for anions over cations obtained from MD simulations do not seem to correspond with a conductive channel (Anishkin and Sukharev, 2004; Sotomayor and Schulten, 2004; Spronk et al., 2006). 
In order to understand the molecular basis of its gating mechanism, more structural and electrophysiological information must be obtained. On the available structure, the $\mathrm{NH}_{2}$-terminus (26 residues) of the channel was not resolved, and the origin of its voltage modulation has not been found yet, even though it has been characterized (Akitake et al., 2005). Having more information about these topics might contribute to fill the gaps that are currently present regarding MscS gating, and it will help to explain the disparities found in molecular dynamics regarding ion conduction (Anishkin and Sukharev, 2004; Sotomayor and Schulten, 2004; Sotomayor et al., 2006). The study of MscS would be of great interest since it belongs to a large and widespread family (Pivetti et al., 2003) that is distributed among prokaryotes, archaea, and plants (Haswell and Meyerowitz, 2006; Kloda and Martinac, 2002b; Nakayama et al., 2007). Therefore, a detailed understanding of the gating mechanism would not be limited to the prokaryotic channel family, but also important clues about how mechanosensation occurs in several other channels.

\section{c) Lipid-Protein Interactions and Channel Activation}

The mechanosensitive channels activation process is affected by changes in tension within the membrane. To understand how these channels work is crucial to understand the meaning of tension. As defined by Laplace's relationship, under patch clamp conditions, tension is defined as $\mathrm{T}=1 / 2 \mathrm{P} . \mathrm{r}$, where $\mathrm{P}$ is the $\mathrm{TM}$ pressure (across the patch) and $\mathrm{r}$ is the radius of the geometric membrane curvature (Moe and Blount, 2005; Sukharev et al., 1999). By measuring MscL open probability while the curvature radius of the patch was monitored, it was possible to prove that changes in the pressure across the patch or changes in radius on their own were not enough to activate MscL rather their 
combination would be more efficient in opening the channel. Moe and Blount (Moe and Blount, 2005) were able to show that the first gating event occurred when the membrane had already achieved $92 \%$ of the smallest radius, but the abrupt change in open probability from 0.5 to 1 only occurred after the patch radius had decreased an extra $8 \%$. MscL open state would be favored by intrinsic membrane curvature changes that can be easily modified by pressure changes across the patch-clamp configuration or through the use of lipid mixtures.

Indeed, electrophysiological evidence suggests that in mechanosensitive channels open probability can be modulated in vitro by the addition of cone-shaped molecules (i.e. charged amphipaths, certain anesthetics, or lysophospholipids) (Maingret et al., 2000; Martinac et al., 1990; Perozo et al., 2002a). The interpretation of amphipathic molecular shape effect is rather complicated because the addition of lysophospholipids generates changes in intrinsic curvature and membrane thickness (Figure I.5). The current hypothesis is based on the release of intra-bilayer lateral pressure as the consequence of shape inequality between bilayer forming phosphatidylcholine and non-bilayer-forming lipids (such as lyso PC). Perozo and colleagues directly tested this hypothesis by complementing the shape effect of phosphatidylethanolamine (a conical lipid that promotes hexagonal phase) with lyso PC (a conical-micelle forming lipid). They measured the conformational changes of an MscL spin-labeled cysteine mutant (in the narrowest position of the pore) reconstituted in DOPC and DOPC/POPE liposomes, and obtained for DOPC/POPE vesicles an activation curve shifted toward higher concentrations of lyso PC than the one obtained for DOPC vesicles alone (Perozo et al., 2002b). 
A

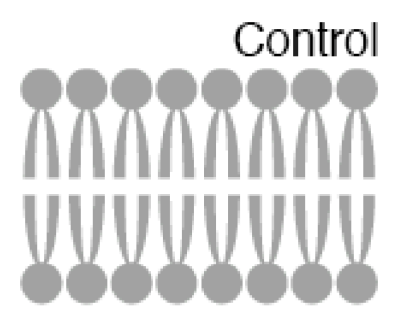

B
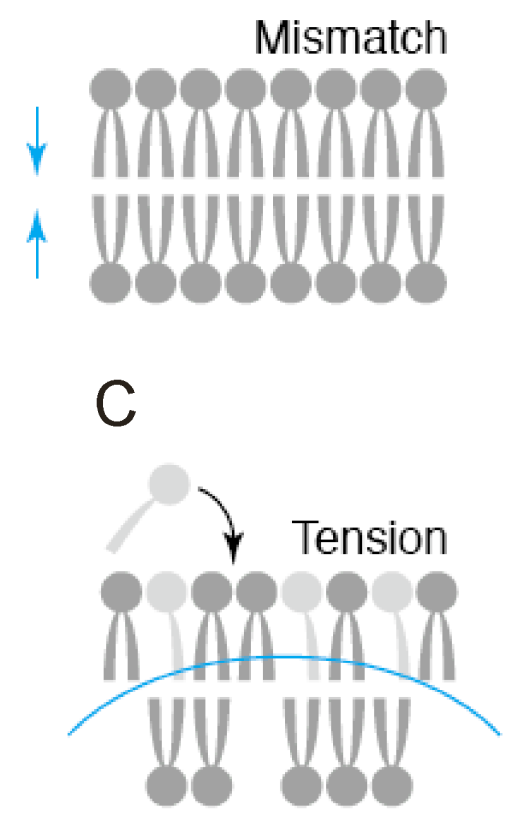

Pressure profile
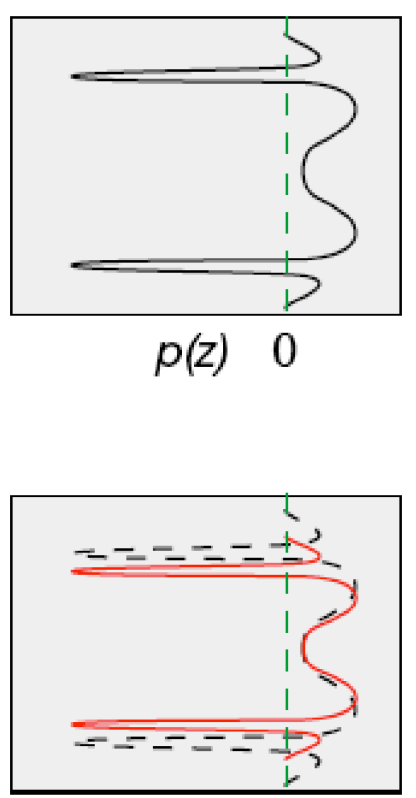

$p(z) \quad 0$

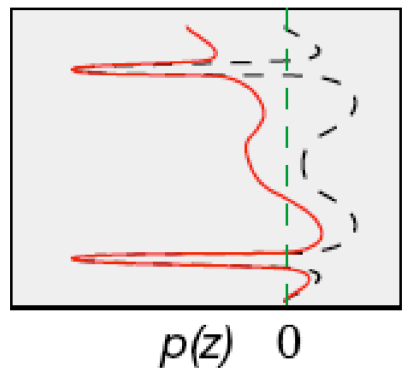

MscL conformation

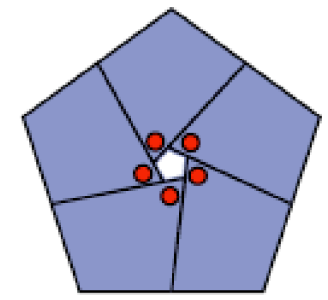

Closed

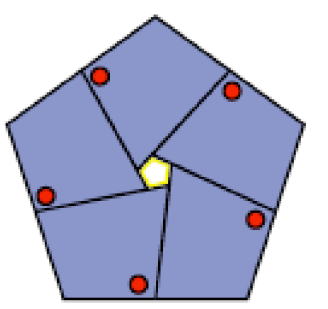

Intermediate?

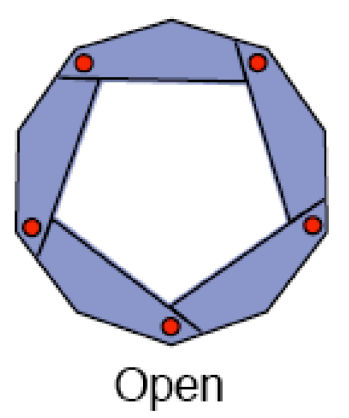

Figure I. 5. Changes in the force profile of the lipid bilayer are the trigger for mechanosensitive channels opening. A) An unperturbed bilayer stabilized the channel in the closed conformation. B) Reconstitution of MscS into bilayers of different thickness compresses/expands the pressure profile and biases the threshold of activation through hydrophobic mismatch, possibly stabilizing an intermediate conformation of the channel. C) Asymmetric incorporation of cone-shaped lipids alters the pressure profile, favoring the fully open state. (Perozo and Rees, 2003). 
It has also been reported in a number of papers that addition of amphiphiles or lyso PCs to membranes containing mechanosensitive channels, significantly lowered the activation threshold (Maingret et al., 2000; Martinac et al., 1990). In the particular case of MscL, externally applied lyso PC strongly favors the open state. The lyso PC effect suggests a second type of mechanosensitivity (Figure I.5), where the asymmetric addition of cone-shaped lipids in one of the membrane leaflets generates a torque that stabilizes MscL in the open state. On the other hand, hydrophobic mismatch alone was unable to open MscL, but decreasing bilayer thickness (by using short acyl-chain lipids) lowered MscL activation energy, stabilizing an intermediate state (Perozo et al., 2002a; Perozo et al., 2002b). These evidences suggest that MscL gating occurs due to changes in the membrane pressure profile in parallel with a protein conformational change.

\section{I.2.2 Site-Directed Spin Labeling and Electron Paramagnetic Resonance}

\section{Spectroscopy}

Site-directed spin labeling and EPR spectroscopy (Altenbach et al., 1990) has emerged as a very powerful approach for studying the structure, dynamics and conformational changes of proteins, particularly for membrane proteins (Fanucci and Cafiso, 2006; McHaourab and Perozo, 2002). The power of this experimental approach comes from the possibility to include a nitroxide group at any given position within the protein by cysteine mutagenesis, and moreover for its sensitivity to changes in environmental parameters, such as dynamics, solvent accessibility, and inter-spin distances (Hubbell et al., 1998).

The EPR spectra enclose information about the motional freedom of the nitroxide spin-label and how it is affected by local steric restrictions. A qualitative analysis of the 
CW-EPR spectra has been used as an empiric measurement of probe dynamics, in which the mobility parameter or $\Delta \mathrm{H}_{0}^{-1}$ (left panel on Figure I.6) represents the inverse of the central resonance width (McHaourab et al., 1996). It is primarily sensitive to the rate of rotational diffusion of the nitroxide (Fanucci and Cafiso, 2006); and therefore, higher values of $\Delta \mathrm{H}_{0}^{-1}$ represent an increase in spin-label motion, while a decrease indicates a reduction in the probe motional freedom (Perozo et al., 1999). For samples containing multiple spin-labels (i.e. homo-oligomeric proteins, labeled ligand-receptor pairs, intrasubunit labeled positions, etc.), the spectral line shape can be affected by the extent of through-space spin-spin dipolar coupling (right panel on Figure I.6). This type of interaction can produce spectral broadening in a distance-dependent manner (Eaton et al., 2005). Using CW-EPR at room temperature, it is possible to measure distances between 7 to $16 \AA$, where collisional spin exchange has high probability of occurring. However, in systems that consist of more than two spin-labels (such as trimers, tetramers, pentamers, and heptamers) the spin-spin interaction cannot be directly translated into quantitative distances. Instead, the broadening of the spectra can be used to estimate changes in overall proximity (Perozo et al., 1998, 1999; Perozo et al., 2002a; Perozo et al., 2001). 


\section{Probe Mobility}

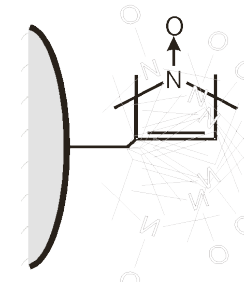

From line-shape analysis

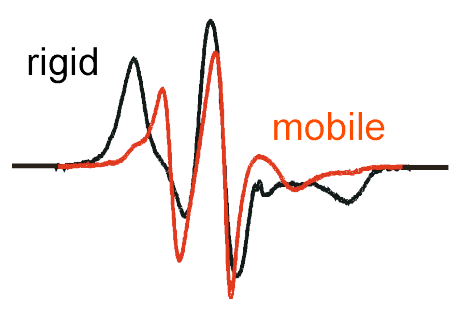

Solvent Accessibility

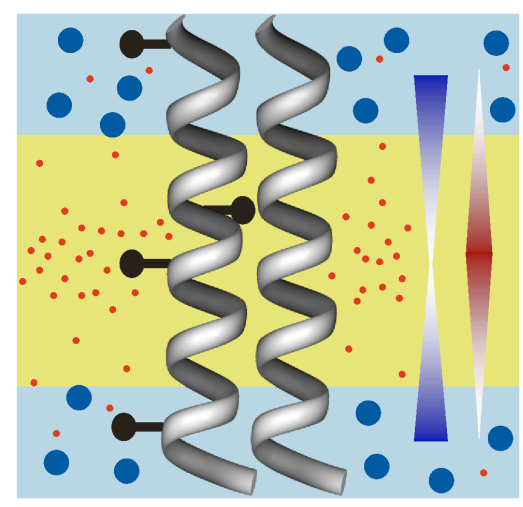

Through collisional relaxation agents

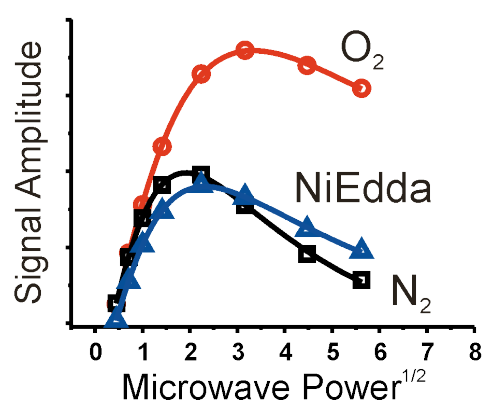

Spin Distances

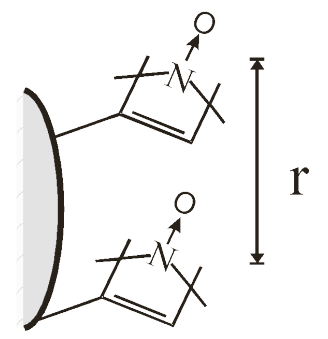

From dipolar broadening:

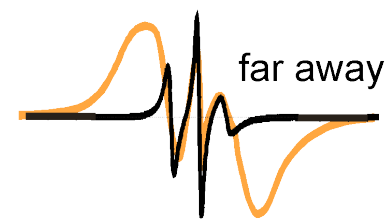

really close

Figure I.6. Structural parameters derived from EPR experiments. Left panel. Dynamic and motional freedom of the probe from spectral-line shape. Top, cartoon representing the mobility freedom of the methanethiosulfonate spin label. Bottom, EPR spectra of two cysteine-labeled residues that are facing different environments (black and red spectra representing buried and exposed positions, respectively). Middle panel. Solvent accessibility through collisional relaxation agents. Top, cartoon depicting two $\alpha$-helices with distinctive labeled positions and the difference in distribution between two spin-label quenchers. Blue spheres represent NiEdda, a polar compound highly soluble in aqueous environments. Red spheres represent $\mathrm{O}_{2}$, which is highly soluble in the non-fatty region of the bilayer. Bottom, a representative power saturation experiment of a membrane exposed spin-labeled residue, in the presence of $\mathrm{O}_{2}$, $\mathrm{NiEdda}$, or $\mathrm{N}_{2}$. Right panel. Cartoon depicting dipolar coupling between nitroxide spin-labels. Bottom, EPR spectra of interacting residues under different protein conformations (i.e. closed and open, apo and bound states, etc). 
Besides probe mobility, other environmental parameters can be obtained from spin-labeled cysteine mutants under CW-EPR. Through power saturation experiments (middle panel on Figure I.6), it is possible to determine the local environment surrounding a specific probe. The accessibility of the nitroxide spin label comes from the enhancement of the $\mathrm{T} 1$ relaxation rate that is produced by collisional exchange with paramagnetic agents (Fanucci and Cafiso, 2006). This type of experiment is based on the saturation dependence of the EPR resonance amplitude as a function of the microwave power. In this study of the membrane protein $\mathrm{MscS}$, we have used two paramagnetic species, molecular Oxygen, a non-polar fast-relaxing paramagnetic reagent that is highly soluble in the hydrophobic core of the membrane, and Nickel-Ethylenediaminediacetic acid (NiEdda), a very polar complex soluble in the aqueous milieu that has about $4.5 \AA$ radius. Apart from these two species we have also used a lipid metal chelate called DOGS-Ni(II)-NTA that places the Nickel at the aqueous membrane interface (Gross and Hubbell, 2002).

Quantitative analysis of the mobility and accessibilities profiles are used to estimate secondary structure from one-dimensional data set, in order to define the limits of a strand or helix. Furthermore, information on the relative conformation of secondary structure elements, domains or whole-subunits can be obtained by examining patterns of change in probe dynamics and solvent accessibility, or changes in distance among spinlabeled residue pairs between domains or subunits. Nonetheless, side-chain rotamer conformations cannot be analyzed given the fact that this is a reporter group technique (McHaourab and Perozo, 2002). In the particular case of membrane proteins, where the complexity is limited by the nature of the bilayer environment, it is possible to construct 
three-dimensional models by combining mobility and accessibility measurements with computational methods. Therefore, we can obtain experiment-based models that represent the actual orientation and position of a protein relative to the membrane in different conformations (Cortes et al., 2001; Perozo et al., 1999; Perozo et al., 2002a). 


\section{I.3 THESIS OUTLINE}

The results in this thesis clarify a number of inconsistencies found in the MscS literature and provide an unambiguous characterization of $\mathrm{MscS}$ in different conformations. The manuscript reports a combination of experimental and computational approaches used to understand the mechanosensitive channel of small conductance, MscS.

In addition, this work presents several methodologies that improved the quality of purified $\mathrm{MscS}$, as well as some strategies to reconstitute it for spectroscopical and functional experiments (Chapter II). These tactics were implemented in the spectroscopical study of $\mathrm{MscS}$ in different conformations (open and close state). In an effort to understand $\mathrm{MscS}$ gating process, information on lipid-reconstituted channels was obtained by means of site-directed spin labeling and CW-EPR. The first step was to establish the overall topology of the resting $\mathrm{MscS}$ in a native-like environment (Chapter III). The global pattern of mobilities and accessibilities were used to refine the $\mathrm{x}$-ray structure to build a three-dimensional model of MscS in the closed state, which had significant differences with the crystal structure of $\mathrm{MscS}$ obtained in a detergent micelle. The second step was to induce and evaluate MscS arrangements upon opening by changing the membrane pressure profile through asymmetric incorporation and distribution of a cone-shaped lipid in the membrane (Chapter IV). Currently, EPR data on the open state are being imposed on MscS closed conformation, so an open state model can be obtained as well. For the first time, there will be enough experimental evidence to understand the molecular mechanism of MscS gating process. 
The patch-clamp experiments (Chapter V) confirmed that MscS is a slightly anion-selective and pressure activated ion channel. In addition, the experiments indicated that $\mathrm{MscS}$ inactivation, rather than activation, is voltage dependent. The experimentally and unambiguously determined properties of $\mathrm{MscS}$ are compared to the outcome of extensive molecular dynamics simulations (made in collaboration with Klaus Schulten laboratory) probing ion conduction through the available crystal structure of MscS. The comparison revealed that the crystal structure of $\mathrm{MscS}$ might not represent a fully open state as originally suggested, since computationally determined ionic currents are too small and selectivity for anions is too high. By site-directed neutralization of charged residues located in the TM domain, in vivo functional assays and patch-clamp experiments it was possible to demonstrate that the origin of the voltage dependence of the inactivation does not come from the transmembrane charged residues. It was also possible to determine the relevance of an inter-subunit salt-bridge in the modulation of the inactivation process, but this interaction is not the origin of the voltage-dependence. So far the entity of the voltage sensor remains unknown.

The findings mentioned above provide a solid experimental base to explore the structure-function relationships of MscS. 
CHAPTER II: An Optimized Purification and Reconstitution Method for MscS

Channel: Strategies for Spectroscopical Analysis

\section{II.1 ABSTRACT}

The mechanosensitive channel of small conductance $(\mathrm{MscS})$ plays a critical role in the osmoregulation of prokaryotic cells. The crystal structure of MscS revealed a homoheptamer with three transmembrane segments and a large cytoplasmic domain. It has been suggested that the crystal structure depicts an open state, but its actual functional conformation remains controversial. In the pursuit of spectroscopical approaches to MscS gating, we determined that standard purification methods yield two forms of $\mathrm{MscS}$, with a considerable amount of unfolded channel. Here, we present an improved high yield purification method based on E. coli expression, optimized to yield approximately $4 \mathrm{mg}$ of a single monodisperse product, and a biochemical characterization of the reconstituted channel. Upon reconstitution into lipid vesicles, MscS is unusually prone to lateral aggregation depending on the lipid composition, particularly after sample freezing. Strategies have been developed to minimize $\mathrm{MscS}$ aggregation in two dimensions for spectroscopic analysis of gating. 


\section{II.2 INTRODUCTION}

Mechanotransduction plays an important role in a wide range of physiological processes such as touch, pain, hearing, balance, proprioception, blood pressure regulation, turgor control in plant cells, and regulation of cell volume. It has been suggested that mechanosensitive channels might have evolved as cellular osmoregulators that sensed changes in the concentration of water across bacteria membranes (Batiza et al., 1999; Blount, 2003; Hamill and Martinac, 2001; Kung, 2005; Martinac, 2001, 2004; Perozo, 2006). Mechanosensitive channels belong to a structurally heterogeneous group, and have been classified according to their function rather than topology. It includes members of the two-pore domain potassium channel (Maingret et al., 1999b; Patel et al., 1998), the amiloride-sensitive sodium channel (Kellenberger and Schild, 2002), the TRP channels (Corey, 2003), and the prokaryotic mechanosensitive channels (Berrier et al., 1996).

The prokaryotic mechanosensitive channels are key components in the emergency response of prokaryotes to sudden osmotic downshock (Batiza et al., 2002; Levina et al., 1999). These channels are classified according to their conductance and to the amount of tension needed to activate them as MscL, MscS, and MscM; for mechanosensitive channel of Large, Small, and Mini conductance, respectively. Particularly, MscL and MscS have been shown to play a critical role in osmoregulation, since cells lacking mechanosensitive channels lyse upon osmotic downshock (Levina et al., 1999). Over the past years, a considerable amount of functional and structural information has been gathered on the prokaryotic mechanosensitive channel family. Electrophysiological and spectroscopical approaches have provided information on their conductance properties 
and activation process (Berrier et al., 1996; Koprowski and Kubalski, 1998; Martinac et al., 1987; Perozo et al., 2002a; Sukharev et al., 1993). The crystal structures of MscL and MscS have revealed their three dimensional structure (Bass et al., 2002; Chang et al., 1998) and a critical starting point for mechanistic interpretations. Still, the gating mechanism in either of these channels is not fully understood.

MscS (Figure II.1.A) belongs to a large and widespread family of membrane proteins (Pivetti et al., 2003), that is distributed among prokaryotes, archaea and plants (Haswell and Meyerowitz, 2006; Kloda and Martinac, 2002a) in which function is defined by interaction with the lipid bilayer, and as such represents an ideal model system to study the biophysical basis of mechanotransduction. Besides, protein-membrane interactions are not unique to mechanosensitive channels since the open probability of several other ion channels is modulated via membrane stretch or membrane composition (Calabrese et al., 2002; Jennings et al., 1999; Laitko et al., 2006; Langton, 1993). A highquality biochemical preparation is required to routinely express and purify (at milligrams levels) MscS from E. coli, which might help increase crystal resolution and allow efforts towards the elucidation of the molecular mechanisms of tension dependent gating. Here, we present a new approach for high expression, and purification of several MscS constructs in different $E$. coli strains. We also present a strategy to reconstitute $\mathrm{MscS}$ in liposomes that avoids its natural tendency to aggregate in the plane of the bilayer. These methodologies will play a key role in our attempts to understand the molecular basis of MscS gating mechanism. 
A

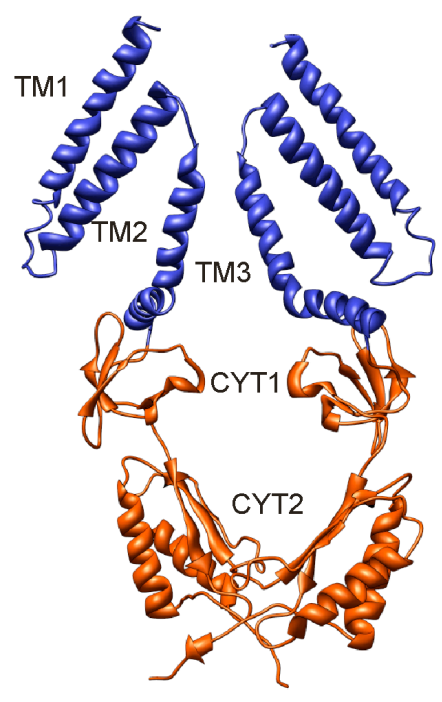

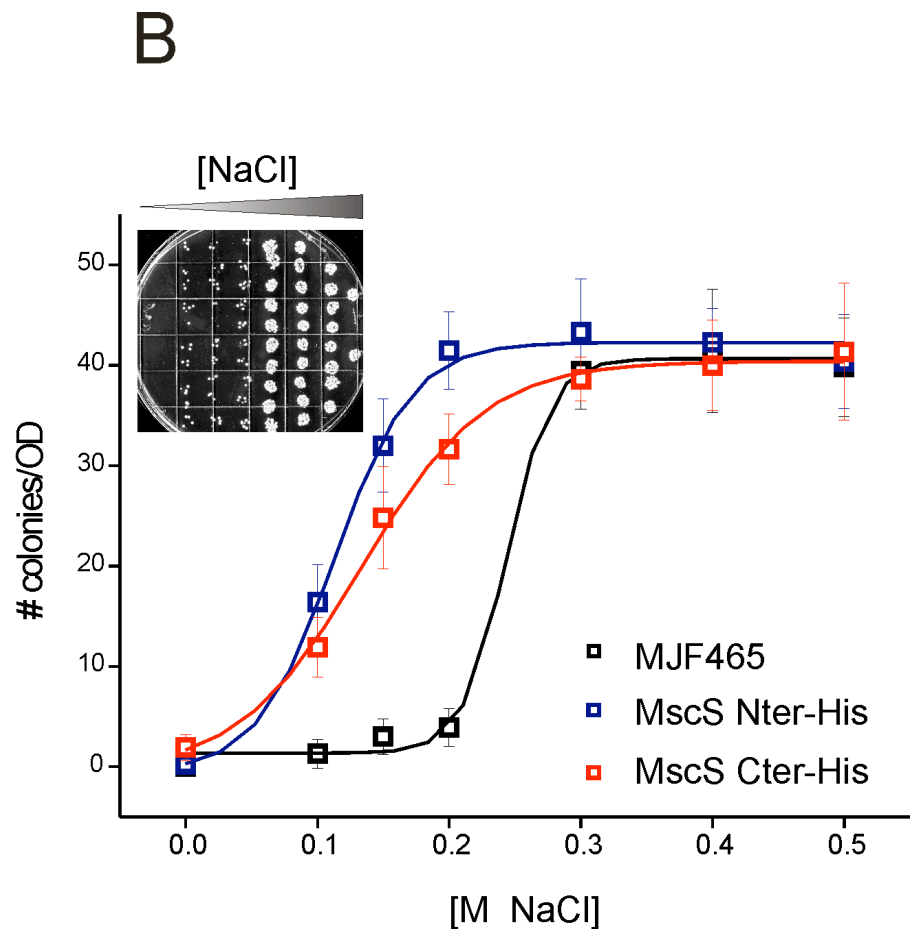

[M NaCl]

Figure II.1. Functional characterization of different MscS constructs. A) Ribbon representation of MscS showing two subunits of the heptamer. TM helices are shown in blue and cytoplasmic (CYT) domains are shown in red. B) Viability assay of MscS constructs upon osmotic downshock, following the protocol of Batiza et al (Batiza et al., 2002). The downshock was made by diluting the cells in various shock media ranging from 0 to $500 \mathrm{mM} \mathrm{NaCl}$. The strain MJF465 (black curve) was used as a negative control since it lacks native mechanosensitive channels. The blue curve represents MscS expressed in $\mathrm{pQE} 32$ with 6 Histidines at the N-termini; and the red curve represents MscS expressed in pQE70 with 6 Histidines at the C-termini. The inset shows a representative result of MJF465 cells upon downshock assay. 


\section{II.3 EXPERIMENTAL PROCEDURES}

\section{II.3.1 Materials}

MJF429 (Frag1, $\Delta y g g B, \Delta k e f A)$ and MJF465 (Frag1, $\Delta m s c L, \Delta y g g B, \Delta k e f A)$ (Levina et al., 1999) were kindly donated by Ian R. Booth from the University of Aberdeen. E. coli Rosetta $^{\mathrm{TM}}$ and the vector pET28a were purchased from Novagen (San Diego, CA). E. coli M15, SG1300 and the vectors pQE32 and pQE70 were bought from QIAGEN (Valencia, CA). The IPTG, and the detergents Fos-Choline $14^{\circledR}$ (solubilization grade) and DDM (solubilization grade) were obtained from Anatrace (Maumee, OH). Talon cobalt resin was bought from Clontech (Mountain View, CA). The reducing agent TCEP was purchased from Pierce (Rockford, IL). The reducing agent TCYP, along with the fluorophores Fluorescein-5-maleimide, and Tetramethylrodhamine-5-maleimide were bought from Molecular Probes (Carlsbad, CA). The spin label was purchased from Toronto Research Chemicals Inc. (North York, On, Canada). The lipids DOPC, POPG, POPE, and E. coli polar lipid extract were bought from Avanti Polar Lipids Inc. (Alabaster, AL). All other reagents were purchased from Sigma or Fisher.

\section{II.3.2 Constructs and Strains}

A $600 \mathrm{ng}$ amount of E. coli genomic DNA was used as a template for PCR. The resultant product ( $846 \mathrm{bp}$ ) was gel purified and cloned in pQE32 and in pET28a, vectors containing a His6 epitope at the N-terminus, and in pQE70 in which the His6 epitope was at the C-terminus. MscS-pQE32 and MscS-pQE70 were used to express the channel in $E$. coli M15, SG1300, MJF429 and MJF465; MscS-pET28 was used for expression in E. coli Rosetta $^{\mathrm{TM}}$. 


\section{II.3.3 Functional Assay}

Downshock assays were developed as described in reference (Batiza et al., 2002), with the following modifications: cultures growing in LB plus $500 \mathrm{mM} \mathrm{NaCl}$ were induced at $\mathrm{OD}_{600}$ of 0.6 with $1 \mathrm{mM}$ IPTG; after 1 hour of induction the cells were adjusted to an $\mathrm{OD}_{600}$ of 1.0, pelleted down and then resuspended in $50 \mu 1$ of fresh LB plus $500 \mathrm{mM}$ $\mathrm{NaCl}$. The downshock was made by diluting the cells 1:40 in various shock media ranging from 0 to $500 \mathrm{mM} \mathrm{NaCl}(\mathrm{pH}$ 7). After 5 minutes the cells were serially diluted and $5 \mu \mathrm{l}$ aliquots were plated on LB agar. Plated cells were grown over night, and the colonies were counted and normalized against the $\mathrm{OD}_{600}$ (measured right before the downshock).

\section{II.3.4 Expression and Purification of MscS in E. coli cells}

Fresh competent cells were transformed with the desired construct, and the transformation mixture was grown over night in the presence of the proper antibiotics. The cells were diluted 1:100 in regular LB media, and grown at $37^{\circ} \mathrm{C}$ up to $1.0-1.2 \mathrm{OD}_{600}$, protein expression was induced with the addition of $0.8 \mathrm{mM}$ IPTG in the presence of $0.4 \%$ glycerol, for 4 hours at $26^{\circ} \mathrm{C}$. The cells were harvested and homogenized (high pressure homogenizer EmulsiFlex ${ }^{\circledR}-\mathrm{C} 3$ ) immediately, in the presence of PBS plus 1mM PMSF (Cortes and Perozo, 1997). The membranes were spun down at $100,000 \mathrm{~g}$ for 30 minutes, and the pellet was resuspended in PBS + PMSF. Solubilization was carried out with $1 \%$ Fos-Choline $14^{\circledR}$ (Bass et al., 2002), in the presence of $10 \%$ glycerol in PBS+PMSF, at $\mathrm{pH} 7.5$, overnight at $4^{\circ} \mathrm{C}$; solubilization with $6 \mathrm{mM}$ DDM resulted in a significantly lower protein yield. This homogenate was spun down at 100,000 $\mathrm{g}$ for 30 minutes, and the supernatant was incubated with cobalt resin for 3 hours at $4^{\circ} \mathrm{C}$. The resin 
was then washed with $1 \mathrm{mM}$ DDM (regardless of initial detergent), $10 \%$ glycerol in PBS buffer $\mathrm{pH} 7.5$, the final wash was made with 5mM imidazole, $1 \mathrm{mM}$ DDM, and $10 \%$ glycerol in PBS buffer $\mathrm{pH}$ 7.5. The channel was eluted with $500 \mathrm{mM}$ imidazole, $1 \mathrm{mM}$ DDM, and $10 \%$ glycerol in PBS buffer $\mathrm{pH}$ 7.5. The typical yield of WT MscS was $\sim 4$ mg for M15, SG1300, and Rosetta ${ }^{\mathrm{TM}}$, and $2 \mathrm{mg}$ for MJF429 and MJF465 per one liter of LB culture. When MscS Cys-mutants were purified for fluorescence or CW-EPR measurements, washing buffers were degassed and contained $0.5 \mathrm{mM}$ TCEP and $0.5 \mathrm{mM}$ TCYP. To check the oligomeric state of the protein, samples were run in a Superdex ${ }^{\mathrm{TM}}$ 200 HR 10/30 (Amersham Biosciences, Sweden) and monitored in an AKTA FPLC system, model P-920, from the same company.

\section{II.3.5 Molecular Mass Determination by Static Light Scattering}

The purified MscS was run on a Superdex ${ }^{\mathrm{TM}} 200$ HR 10/30 column coupled to a light scattering system, refractive index (Wyatt Technology Corp.) and UV (Shimadzu) detectors. The system was equilibrated with a buffer containing $1 \mathrm{mM}$ DDM, $1 \mathrm{mM}$ EDTA, $150 \mathrm{mM} \mathrm{NaCl}$, and $20 \mathrm{mM}$ Hepes, at $\mathrm{pH}$ 7. The light scattering was measured using a linearly polarized GaAs laser at $\lambda=690 \mathrm{~nm}$ from $57^{\circ}$ to $126^{\circ}$ by 9 detectors. Increment of refractive index over increment of protein concentration for proteindetergent complex, $(\mathrm{d} n / \mathrm{d} c)_{\text {pd }}$ was first determined using the following equation:

$$
(\mathrm{d} n / \mathrm{d} c)_{\mathrm{pd}}=k_{2} \varepsilon R I / U V
$$

where $\varepsilon$ is the extinction coefficient of the protein at $\left.280 \mathrm{~nm}_{\left(\mathrm{mg}^{-1} \mathrm{ml} \mathrm{cm}\right.}^{-1}\right), R I$ is the value of refractive index, and $U V$ is the absorbance at $280 \mathrm{~nm}$. The $k_{2}$ value was determined using a standard curve from soluble standard proteins (bovine albumin, $\beta$ - 
amylase, and apoferritin F; Sigma) whose $\mathrm{d} n / \mathrm{d} c$ is constant at $0.187 \mathrm{ml} \mathrm{g}^{-1}$. Using the experimentally determined $(\mathrm{d} n / \mathrm{d} c)_{\text {pd }}$ value $(=0.24)$, the protein molecular weight was determined from the Debye plot where the intensity of light scattering is plotted against the scattering angle (ASTRA software, Wyatt Technology Corp.).

\section{II.3.6 MscS Reconstitution}

After purification, $\mathrm{MscS}$ was reconstituted in pre-formed liposomes following protocol described in reference (Perozo et al., 1998). For electrophysiological measurements MscS was reconstituted in a protein to lipid ratio of 1:200 (mass to mass). For CW-EPR and FRET experiments, the reconstitution ratio was of 1:750 (mol to mol). For patch-clamp experiments the lipids were resuspended in buffer HEPES, pH 7.5, for fluorescence and EPR measurements the lipids were resuspended in PBS, pH 7.5. In either case, the dilution method (to decrease the DDM concentration below its CMC), in presence of Bio-beads, was used to incorporate the channels into the liposomes. After three to ten hours of incubation with bio-beads (unless explicitly stated), the proteoliposomes were pelleted down at 100,000 $\mathrm{g}$, resuspended and used for spectroscopic measurements; for electrophysiological measurements they were subjected to a dehydration-rehydration cycle as previously described (Cortes et al., 2001; Sukharev, 2002).

\section{II.3.7 Liposome Patch-Clamp}

Single channel currents were done in the inside-out configuration under symmetrical (200 mM KCl, $90 \mathrm{mM} \mathrm{MgCl}, 10 \mathrm{mM} \mathrm{CaCl}_{2}$, and 5mM HEPES) and asymmetrical conditions $(100 \mathrm{mM} \mathrm{KCl}$ in the pipette and $300 \mathrm{mM} \mathrm{KCl}$ in the bath, all of the other salts were kept constant), at $\mathrm{pH}$ 6. The pipettes were obtained from glass 
capillaries, and were fire polished before used, until it was reached a resistance between 2 and $2.5 \mathrm{M} \Omega$. Negative pressure on the patch was obtained by applying suction through a syringe and monitored with a homemade piezo-electric pressure transducer. Single-channel and macroscopic currents were recorded with a DAGAN 3900 patchclamp amplifier, and currents were sampled at $10 \mathrm{KHz}$ with an analog filter set to $2 \mathrm{KHz}$. Single channel analyses were done using pCLAMP9 (Axon Instruments) (Sotomayor et al., 2007).

\section{II.3.8 EPR Spectroscopy and Analysis}

MscS-Cys mutants were purified and spin-labeled using 1:10 molar excess of the label. After reconstitution, their spectra were obtained at room temperature in a Bruker EMX X-band EPR spectrometer, and the mobility of the probe was obtained from the inverse central line width of the EPR spectrum $\left(\Delta \mathrm{H}_{0}^{-1}\right)$ (Perozo et al., 1998).

\section{II.3.9 Fluorescence Spectroscopy}

For FRET experiments, single MscS-Cys mutants were purified and labeled with Fluorescein and Tetramethyl-rhodamine, at a 1:10 molar ratio (monomer:fluorophore) following reference (Cuello et al., 2004). After the samples were reconstituted, measurements were done in a Shimadzu 1501 Spectrofluorimeter. 


\section{II.4 RESULTS}

\section{II.4.1 Functional Characterization of His-tagged MscS constructs}

MscS was cloned in several vectors that mainly differ in the position of the HisTag. To test the functional behavior of these constructs, a downshock assay was carried out in MJF465, an E. coli strain that lacks genomic MscL, MscS, and MscK. Figure II.1.B illustrates a typical survival experiment (Batiza et al., 2002; Levina et al., 1999) in which the cells are exposed to a sudden reduction of the external osmolarity. MJF465 was used as a negative control because it does not survive under extreme hypotonic conditions $\left([\mathrm{NaCl}]_{1 / 2}=0.24 \pm 0.01\right.$, black trace). We did not found major differences in the mid-activation point of $\mathrm{MscS}$ with 6 Histidines at the N-terminus $\left([\mathrm{NaCl}]_{1 / 2}=0.11 \pm\right.$ 0.005 , blue trace) and $\mathrm{MscS}$ with 6 Histidines at the C-terminus $\left([\mathrm{NaCl}]_{1 / 2}=0.13 \pm\right.$ 0.007, red trace); both of the constructs were able to protect the cell against hypo-osmotic challenge.

\section{II.4.2 Oligomeric Behavior of MscS under various Purification Conditions}

The purification methodology presented here enables production of $\mathrm{MscS}$ at milligram scale in detergent solutions. In an effort to develop a one step purification method suitable for labeling and spectroscopy studies, we tested the procedures already available in the literature. Using the protocol previously described by Bass et al (Bass et al., 2002) we were unable to obtain single mono-disperse peaks by the exclusion chromatography (skipping the anion exchange), as shown in Figure II.2.A (red trace, Nter His Crystal refers to the conditions that were used for crystallography). The purification protocol of Sukharev (Sukharev, 2002), which includes solubilization with OG and purification with a mixture of OG-asolectin, yielded unexpectedly low amounts 
of the putative heptamer (see column labeled "OG purification" in Figure II.2.C) and the chromatogram showed additional peaks (data not shown).

Our failure to generate a consistent monodisperse peak with either of these methods prompted us to pursue an alternative protocol. Cultures were grown in LB media (instead of Terrific Broth media) at $37^{\circ} \mathrm{C}$ up to $1.0-1.2 \mathrm{OD}_{600}$, induction of MscS was done with $0.8 \mathrm{mM}$ IPTG in the presence of $0.4 \%$ glycerol at $26^{\circ} \mathrm{C}$ (different IPTG and glycerol concentration highly reduced the protein yield). Cells were homogenized after 4 hours of induction, a key step to increase the yield of the monodisperse peak. Glycerol $(10 \%)$ was used in the purification buffers to increase the amount of functional channel. No significant differences were observed between cold purifications and room temperature ones. Finally, although gel filtration chromatography on Superdex 200 can be used as an additional clean-up step, we have found this not to be necessary unless we are evaluating a new condition or construct.

Once we obtained a single mono-disperse peak of $\mathrm{MscS}$ from the same construct that was used for the crystal trials (Figure II.2.A; black trace, N-ter His), the same purification protocol was used for the construct with the His-Tag at the C-terminus. Surprisingly, the main peak of the C-ter His construct (Figure II.2.B; $12.6 \mathrm{ml}$ elution volume, red trace) was significantly shifted from that of the N-ter His (Figure II.2.A, B; $11.3 \mathrm{ml}$, black trace) even though the latter has only 12 additional residues. Both constructs were quite stable in solution even after several weeks of storage at $4^{\circ} \mathrm{C}$, however the $\mathrm{N}$-ter His construct could be stored up to 3 months, whereas the C-ter His unfolds after 4 weeks (data not shown). The long stability of both constructs makes them suitable for crystal trials. 
A summary of all the different cells, constructs, detergents and conditions tried for MscS purification is shown in Figure II.2.C. The efficiency of each condition was determined by comparing the relative amount of large oligomer (presumably heptamer) obtained per preparation. We found that freezing the cells (not the membranes), longer inductions times, or even inducing in the presence of $500 \mathrm{mM} \mathrm{NaCl}$ diminished the amount of large oligomer peak (gray bars). Unexpectedly, we could not get a stable protein preparation when we solubilized and purified our constructs with OG, a detergent widely used in the solubilization of integral membrane proteins and also used for other MscS construct as previously described (Sukharev, 2002). Solubilization with FosCholine- $14^{\circledR}$ or DDM yields, in both cases, a very stable preparation; the only difference being the amount of protein obtained (twice as much for Fos-Choline- $14^{\circledR}$ ). The best results regarding oligomeric stability and protein yield were obtained with systems where E. coli was tightly regulated (pET and $\mathrm{pQE}$ systems), and no protein leak was present (data not shown).

Figure II.2.D shows the SDS-PAGE profile corresponding to the first peak in the gel filtration chromatogram (11.3 ml elution volume in Figure II.2.A). Four major bands, possibly corresponding to the heptamer $(\sim 200 \mathrm{kDa})$, trimer $(\sim 75 \mathrm{kDa})$, dimer $(\sim 50 \mathrm{kDa})$, and monomer $(\sim 25 \mathrm{kDa})$ of $\mathrm{MscS}$ were observed. At $25 \mathrm{kDa}$, MscS in SDS-PAGE gels runs smaller than its theoretically predicted molecular weight $(31 \mathrm{kDa})$. This behavior was previously reported by a number of labs (Miller et al., 2003a; Miller et al., 2003b; Schumann et al., 2004; Sukharev, 2002). It is noteworthy that MscS, after purification, has some level of resistance to SDS, a strong anionic detergent that promotes oligomer dissociation in most membrane proteins. When the sample corresponding to the second 
peak in Figure II.2.A (13.8 ml elution volume) was run only bands that might correspond to the dimer and the monomer were observed (data not shown). The difference between the two fractions is not due to proteolysis, since mass spectrometry (MS/MS) showed that there were no changes in amino acid content for the monomer.

To further evaluate the hydrodynamic behavior of the purified protein, we used light scattering/refractive index analysis to accurately determine the absolute molecular weight of heptameric MscS, under native conditions. The sample corresponding to the $11.3 \mathrm{ml}$ fraction of the Superdex (Figure II.2.A) was homogenous in size and its mass was of $220 \mathrm{KDa}$. This measurement indicates that we have the proper assembly for the heptamer since the theoretical molecular weight of the monomer is 30,896 Da (Figure II.3). On the other hand, the refractive index and UV values for the $13.8 \mathrm{ml}$ sample (Figure II.2.A) were off and close to the detergent micelle peak (Strop and Brunger, 2005), so that we could not precisely determine its exact mass. However, a rough estimate about $50 \mathrm{KDa}$ suggests the presence of a mixed population of monomer, dimer, and micelle. We have found that, independently of the construct and the strain, the second peak (13.8 ml, Figure II.2.A) essentially disappears when the induction temperature is $26^{\circ} \mathrm{C}$, IPTG levels are less than $1 \mathrm{mM}$, glycerol is kept throughout the induction and purification, induction time does not exceed 5 hours, and more importantly, the cell rupture is done immediately after cells harvesting. We found that some cysteine mutants promote dissociation of the heptamer into an unfolded channel, particularly cysteine substitution on the TM3 segment (position 93 to 101, 107 and 115) which are located along the channel seven-fold axis of symmetry. 

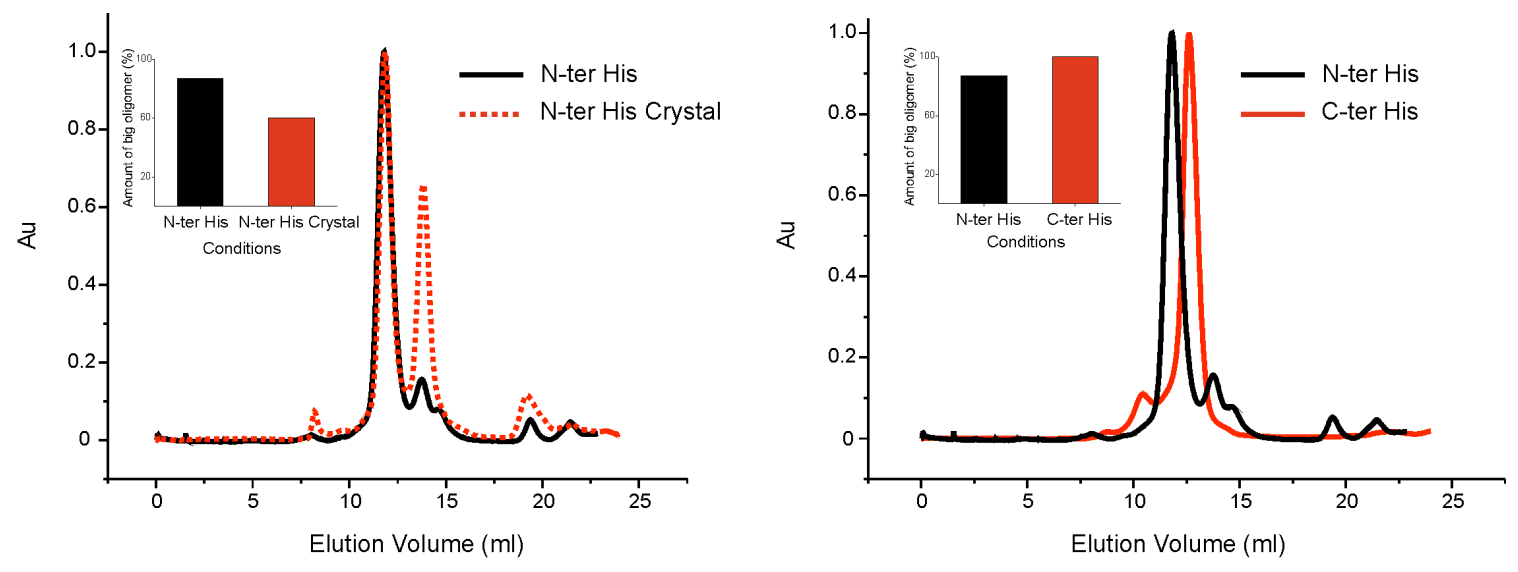

C
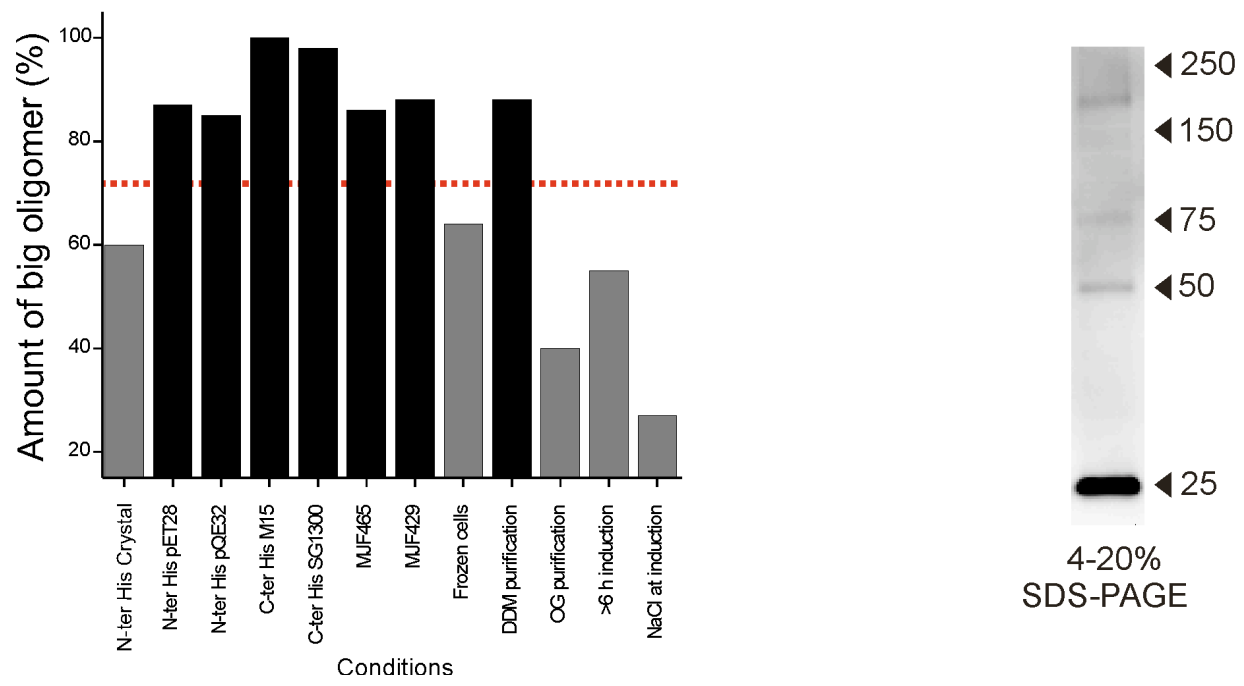

$4-20 \%$

SDS-PAGE

Figure II.2. Evaluation of different $\mathrm{MscS}$ constructs and purification conditions. A) Gel filtration chromatogram of MscS-pET28 expressed in Rosetta cells. Comparison of the purification of MscS constructs containing 6 His plus 12 amino acid residues at the N-termini (pET28 in Rosetta cells). The black curve (N-ter His) represents the conditions were $\mathrm{MscS}$ is induced with addition of $0.8 \mathrm{mM}$ IPTG, $0.4 \%$ glycerol at $25^{\circ} \mathrm{C}$ for 4 hours; and the purification buffers containing $10 \%$ glycerol. The red dashed line (N-ter Crystal) corresponds to $\mathrm{MscS}$ induction with $1 \mathrm{mM} \mathrm{IPTG}$ at $37^{\circ} \mathrm{C}$ for 2 hours. The inset figure represents the amount of putative heptamer per preparation that was obtained for both conditions. B) Comparison of two MscS constructs using the first condition described above (in both cases). The black curve represents the construct with 6 His plus 12 amino acid residues at the N-termini, and the red curve represents the one with 6 His at the C-termini (pQE70 in M15 cells). The inset shows the amount of putative heptamer per preparation that was obtained for both constructs. C) Yield of purified oligomeric $\mathrm{MscS}$ obtained from the expression in different $E$. coli cells, and from variations in the purification protocol, evaluated from the elution time in a gel filtration column. The red dashed line denotes those conditions that give less than $70 \%$ of high oligomer. D) Oligomeric behavior of purified $\mathrm{MscS}$ shown in SDS-PAGE gel. 4-20\% gradient gel shows four possible oligomeric states of MscS after purification; molecular weight $(\mathrm{kDa})$ markers are shown on the right. 


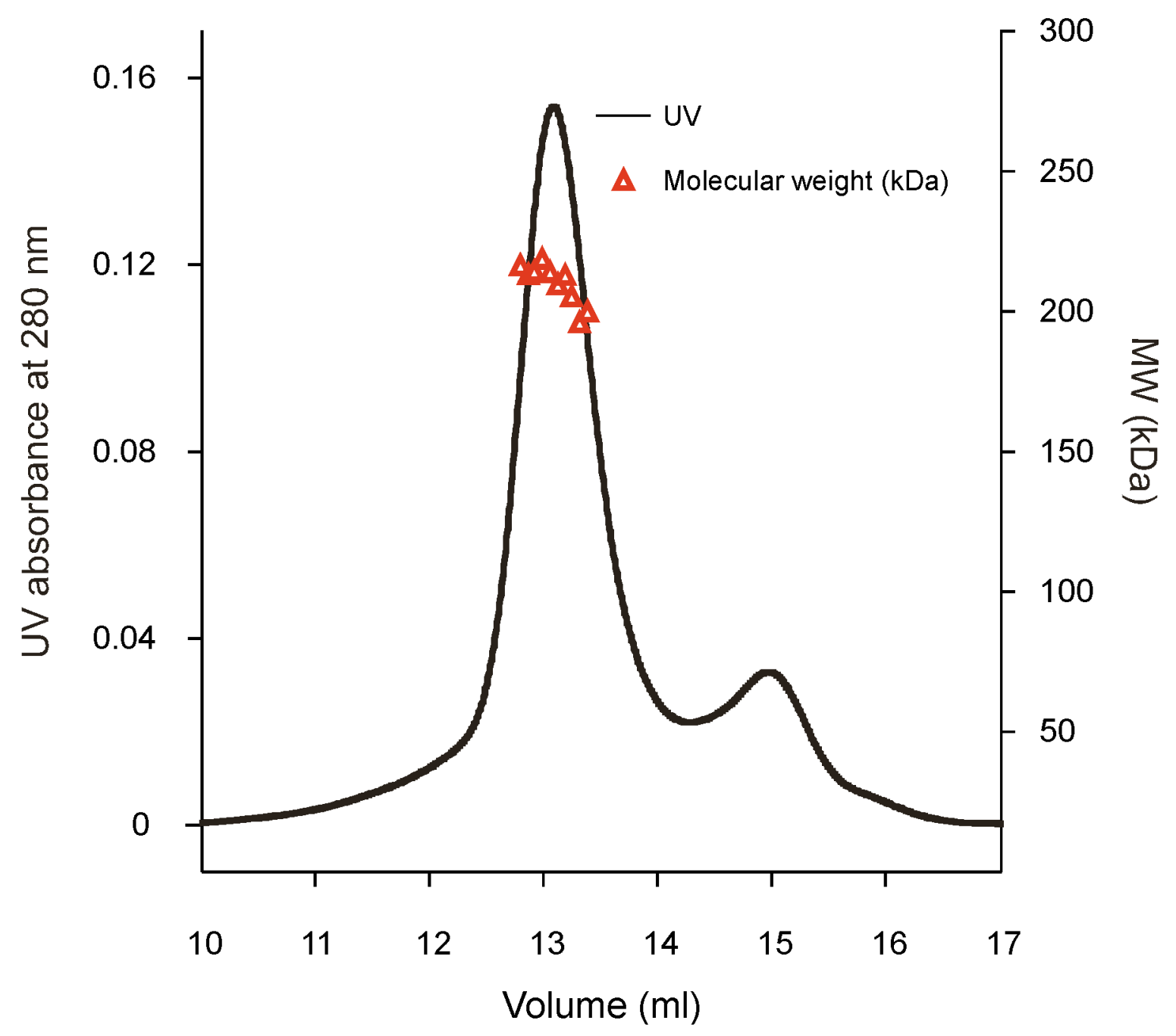

Figure II.3. Molecular mass determination of purified MscS through ASTRA software analysis (Wyatt Technology Corp.) of SEC-LS/UV/RI data. Calculated molecular weight value for heptameric MscS (triangles) is plotted. The solid line corresponds to the UV trace of MscS eluting from the SEC column. 


\section{II.4.3 Functional Characterization of Purified Channel}

We assessed MscS functionality after lipid reconstitution using the patch clamp technique. Gel filtration fractions from the corresponding to the heptamer and the small oligomer were reconstituted in E. coli polar lipids, at a 1:200 protein to lipid ratio (mass:mass). For the $11.3 \mathrm{ml}$ peak, shown in Figure II.2.A, (heptamer, Figure II.4.B) we found the same conductance reported elsewhere $(1 \mathrm{nS})$ in symmetric and asymmetric conditions (Akitake et al., 2005; Sotomayor et al., 2007; Sukharev, 2002; Sukharev et al., 1993). However, we were unable to detect current activity out of the reconstituted 13.8 $\mathrm{ml}$ peak (Figure II.2.A), even though the protein was well reconstituted as suggested by delipidation experiments (data not shown). These results imply that the second peak (Figure II.2.A) derives from an unfolded or partially assembled channel (Figure II.4.C).

\section{II.4.4 Two-Dimensional Aggregation of Reconstituted MscS}

For membrane proteins aggregation is a critical issue, since spectroscopic measurements of local dynamics and solvent accessibility are dependent on the channel being monodisperse on the plane of the bilayer. Two-dimensional aggregation has been reported for ion channels (Cuello et al., 2004; Encinar et al., 2005) and other membrane proteins (Farahbakhsh et al., 1992; McHaourab et al., 1994). To check if MscS tends to aggregate in DOPC membranes we mutated to cysteine and spin-labeled 21 residues (Figure II.5.A) on the TM1 segment (the most exposed TM segment) in order to analyze its mobility profile. Figure II.5.B clearly shows the differences found in local dynamics between the peripheral TM segments in MscL (TM2) (Perozo et al., 2001) and MscS (TM1). The difference in average mobility for both TM segments ( 0.16 for MscS, and 0.22 for MscL) suggests that there is a limited lipid/protein interface for MscS, typical of 
aggregated samples (Cuello et al., 2004). MscS aggregation was also apparent when we compared the present data set with peripherally-located TM segments of ion channels known to be monodisperse, such as TM1 in KcsA (Perozo et al., 1998), and S3-S4 in KvAP (Cuello et al., 2004).

In order to decrease aggregation in DOPC vesicles we included in the mixture POPG, reported to reduce aggregation in KvAP proteoliposomes (Cuello et al., 2004). Addition of POPG in the liposomes (6 DOPC : 1 POPG) resulted in more mobile EPR spectra (Figure II.5.C). When we plotted the mobility profiles for MscS reconstituted in DOPC (gray trace) and in DOPC:POPG (black trace), there were large differences in spin label dynamics (Figure II.5.D), and the average mobility for the latter was similar to the averages reported for other peripheral TM segments. The fact that the average mobility is high, with few motionally restricted positions indicates that this TM segment is not tightly packed against the rest of the protein. 

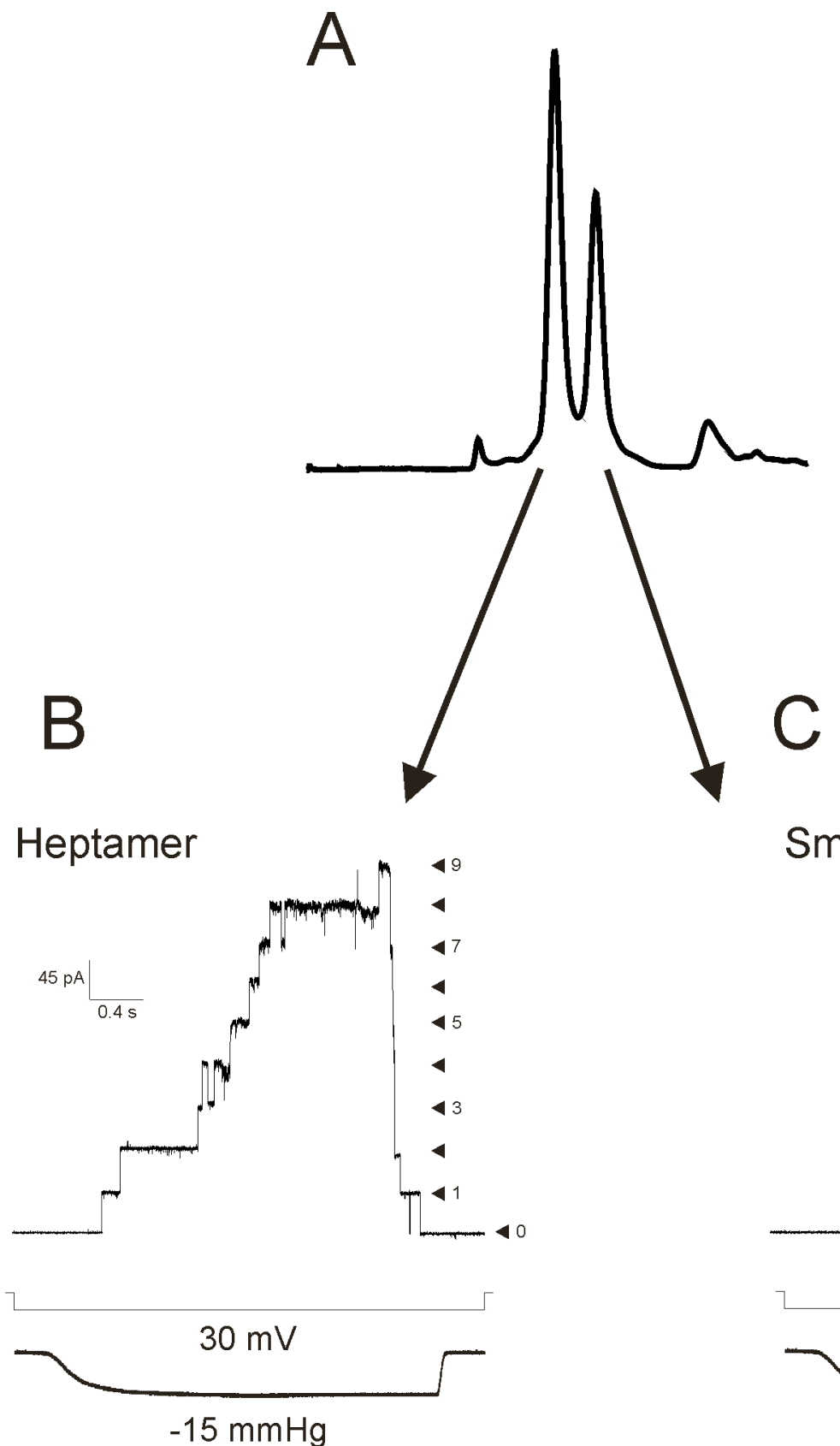

Small Oligomer

Figure II.4. Single channel records from purified $\mathrm{MscS}$ in asymmetric solutions (100 mM KCl pipette/300 mM KCl bath, $\mathrm{pH} \mathrm{6.0);} \mathrm{channel} \mathrm{activity} \mathrm{was} \mathrm{elicited}$ applying negative transbilayer pressure under patch clamp conditions. A) Elution profile of $\mathrm{MscS}$ when the two kinds of oligomers are present in the purification. The arrows point the fractions that were reconstituted in preformed liposomes. B) Representative single-channel traces of purified and reconstituted heptameric MscS in liposomes made with $E$. coli polar lipids in a protein to lipid ratio of 1:200 (mass to mass). C) Reconstitution of small oligomer yields no activity under the same conditions as in $\mathrm{B}$. 
A

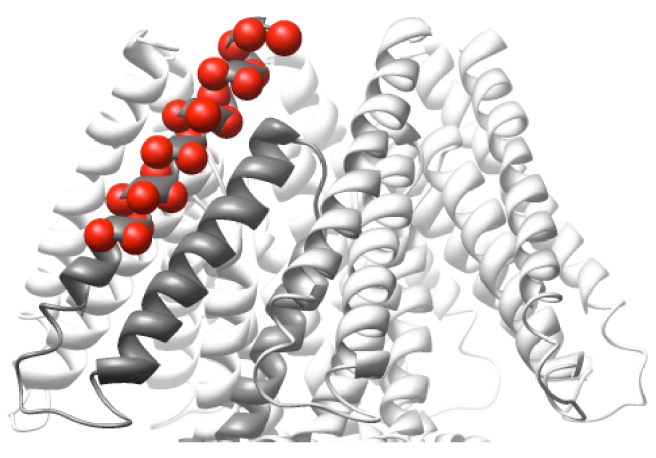

C

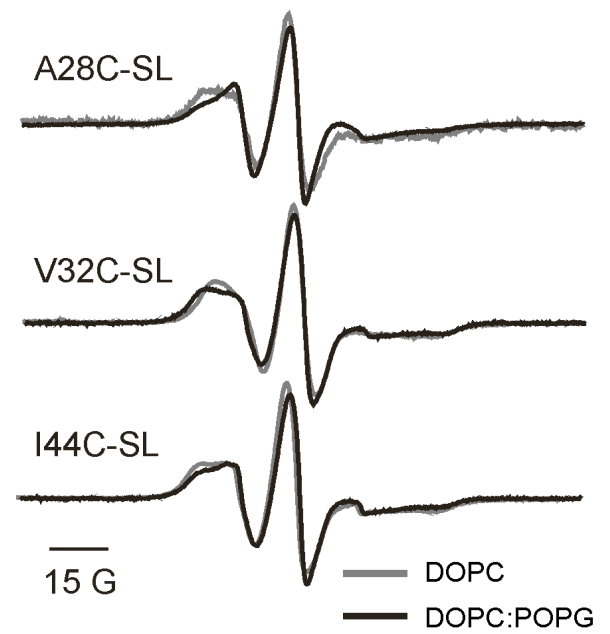

B

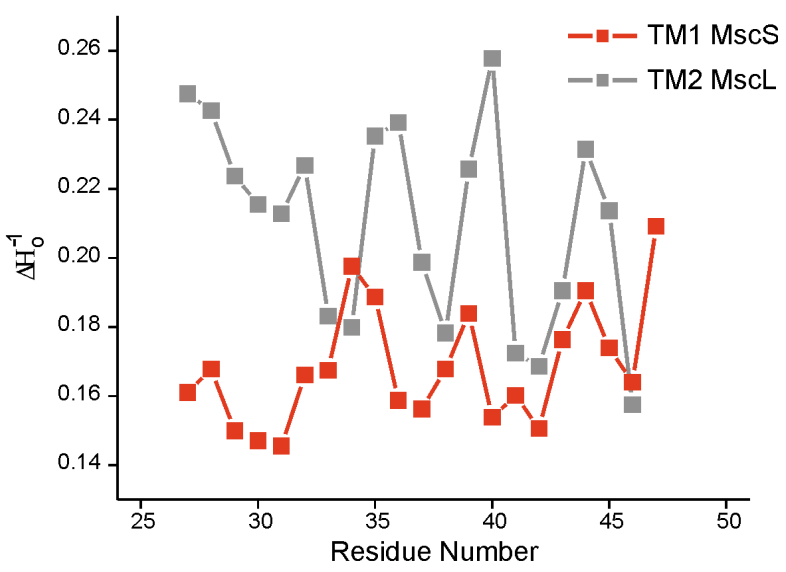

D

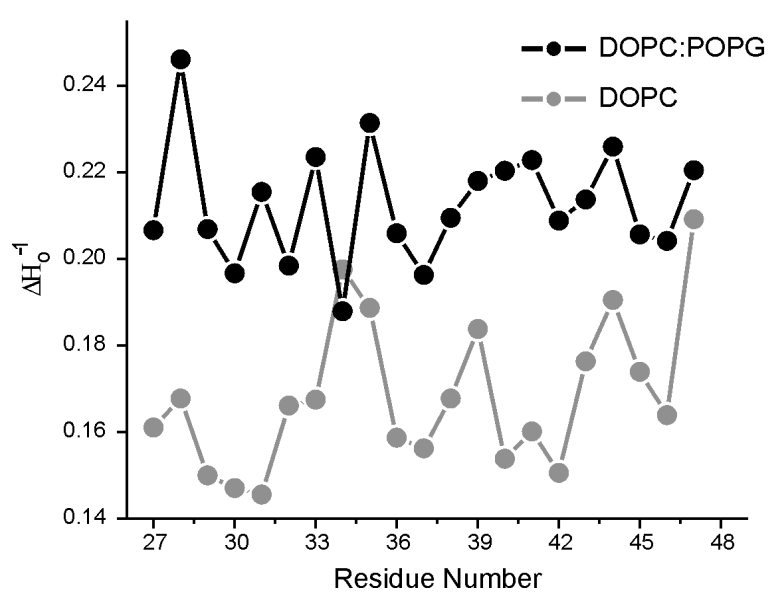

Figure II.5. MscS mobility profiles monitored by CW-EPR from spin labels attached to Cys-residues in the TM1. A) Ribbon diagram of the TM segments of MscS showing in red spheres the TM1 residues that were mutated to Cysteine and spin-labeled. B) Aggregation was detected comparing the mobility $\left(\Delta \mathrm{H}_{\mathrm{o}}^{-1}\right)$ profiles obtained for TM1 in MscS (red trace) and TM2 in MscL (gray trace); both channels were reconstituted in DOPC vesicles. C) Representative first derivative EPR spectra of spin-labeled mutants (A28C, V32C, and $\mathrm{I} 44 \mathrm{C}$ ), reconstituted in DOPC (gray spectra), and in DOPC:POPG liposomes (3:1 ratio, black spectra). D) Mobility $\left(\Delta \mathrm{H}_{\mathrm{o}}{ }^{-1}\right)$ profiles comparison between MscS reconstituted in DOPC:POPG (black trace) and in DOPC vesicles (gray trace). 
We further investigated this phenomenon using reconstituted channels labeled with either fluorescein or tetramethyl-rhodamine, at positions that are putatively exposed to the membrane (Figure II.6.A). When these two-labeled populations are mixed before reconstitution, channels that aggregate in liposomes should produce a strong FRET signal but monodisperse channels should not. Using this readout, we determined that aggregation is critically dependent on the type of lipid used (Figure II.6.B), as well as on the protein-to-lipid ratio of the reconstitution, since a ratio larger than 1:750 (mol:mol) produced aggregation (data not shown). As expected from the EPR results, MscS reconstituted in DOPC membranes (PC, black trace) shows significant FRET signals within 3 hours at room temperature, and are fully aggregated overnight. The presence of anionic lipids in the mixture is required, since DOPC:POPE (PCPE, blue trace) helps reduce the aggregation, while DOPC:POPG (PCPG, red trace) proteoliposomes did not show aggregation at all, unless the samples were frozen (Figure II.6.B-C). Freezing the samples promoted aggregation, under most conditions, independently of the lipid mixture used in the reconstitution. FRET signals were also found after reconstitution in E. coli polar lipids extract (green trace), but not as high as the DOPC signal. 


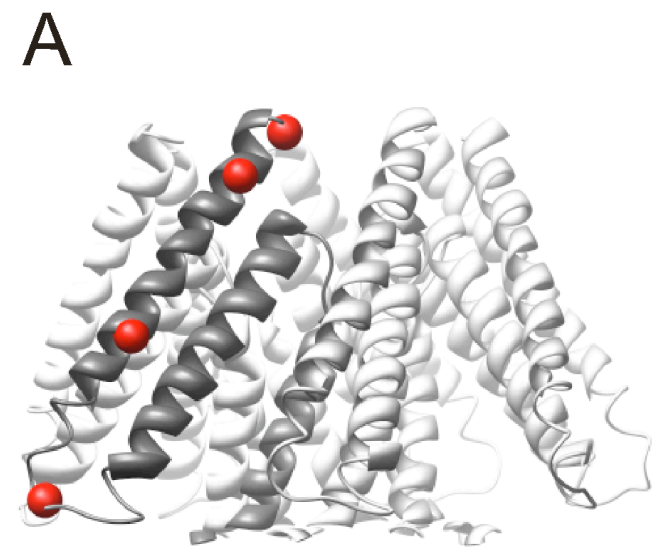

B
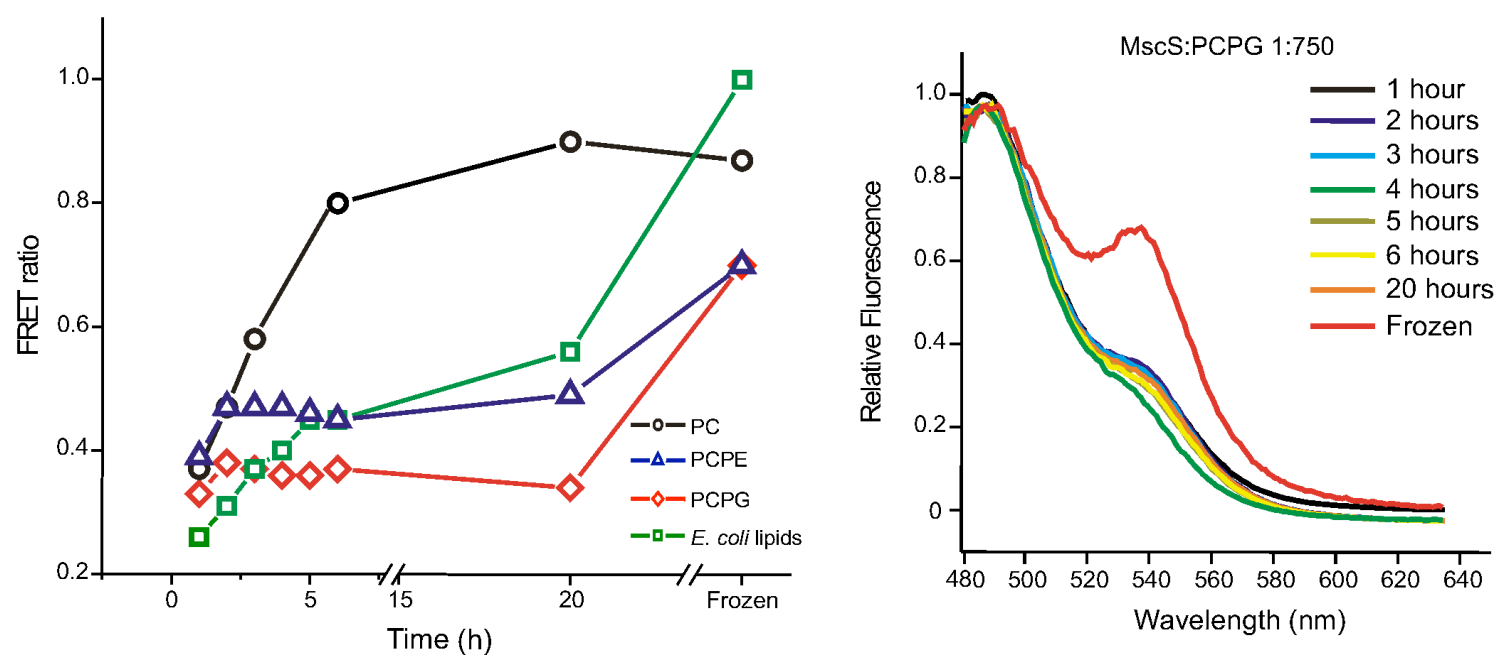

Figure II.6. MscS two-dimensional aggregation in liposomes measured by fluorescence energy transfer (FRET), after co-reconstitution of channels labeled with fluorescein and tetramethyl-rhodamine. Excitation was at $491 \mathrm{~nm}$ and emission collected from $480 \mathrm{~nm}$ to $660 \mathrm{~nm}$. A) Ribbon diagram of the TM segments of MscS showing in red spheres the Cys-residues that were labeled with two different fluorophores (Y27C, V32C, R46C, and R59C). B) Aggregation time course in several system of lipids, DOPC (PC, black trace), DOPC:POPE (PC:PE, blue trace), DOPC:POPG (PC:PG, red trace), and E. coli polar lipid extract (E. coli lipids, green trace). C) Aggregation profile for $\mathrm{MscS}$ in DOPC:POPG, reconstituted in 1:750 protein to lipid molar ratio. Measurements were done at different times after reconstitution, and also after freezing the reconstituted samples. 


\section{II.5 DISCUSSION}

Prokaryotic mechanosensitive channels represent a unique model system to study the molecular basis of mechanotransduction and the role of lipid-protein interactions in membrane protein function. With recent advances in membrane protein crystallization, structures are being obtained at unprecedented rate. Yet as it has been demonstrated, complementary dynamic information is required to ultimately understand membrane proteins functional mechanism, in addition to the structure (Perozo et al., 2002a; Perozo et al., 2002b).

Our first step prior purification was to test the function of our constructs. Even though both constructs were functional, the ability of the C-terminal His-tag construct of MscS to protect the cell from the downshock seems to be lower than that of the construct with the N-terminal His-tag. A study by Schumann and collaborators (Schumann et al., 2004) on the carboxy-terminal showed that alterations on this domain affect the recovered activity of $\mathrm{MscS}$ after the first pulse of tension, under path clamp conditions. The mechanism by which the presence of the histidines at the end of the barrel alters the response of MscS to sudden osmotic changes remains unknown.

Here, we have developed and optimized a one step protocol to routinely purify high quantities of functional MscS. Independently of the construct or the E. coli strain, we found that the key steps to obtain a single monodisperse peak of heptameric MscS are: (1) low temperature induction; (2) low IPTG concentration; (3) addition of glycerol during expression and subsequent purification; (4) short induction times; and (5) cell rupture immediately after harvesting. An important feature of this purification protocol is 
that the channel remained monodisperse after several weeks of storage at $4^{\circ} \mathrm{C}$, and the purification tags did not interfere with the assembly or oligomeric state of $\mathrm{MscS}$.

However, we were surprised by the small amount of heptamer obtained when the cells were induced in the presence of $0.5 \mathrm{M} \mathrm{NaCl}$. Stokes et al (Stokes et al., 2003) were able to measure an increment of expression on genomic MscS and MscL when the cells were grown in high osmolarity conditions. In our hands, while yield increased, only $25 \%$ out of the total amount of channel expressed was in the heptameric form (Figure II.2.C). This might be due to the fact that with $\mathrm{NaCl}$ the stress sigma factor $\mathrm{RpoS}$ is up regulated (Stokes et al., 2003). Thus, by increasing the amount of genomic MscS in the preparation along with the increment of plasmidic MscS (IPTG-inducible), a heterogeneous population of channels decreased the yield of total heptamer.

In spite of its low yield, expressing MscS in E. coli strains such as MJF455 or MJF465 is an important requirement to obtain a homogenous preparation of channels suitable for crystallization. Since electrophysiological analysis have suggested that each E. coli cell expresses about $140-210 \mathrm{MscS}$ monomers (Stokes et al., 2003); it is very likely that the MscS population used in the crystal trials was a mixed one, with the contribution of channels with histidine tag coming from the plasmid and without tag coming from the genome.

Previous electrophoretic analysis of membrane preparations and purified channel showed that MscS monomer migrates as a 25-30 kDa band (Miller et al., 2003a; Sukharev, 2002), and that the heptamer can only be observed in the presence of chemical cross-linkers, or using LDS detergent as recently shown in ref. (Akitake et al., 2007b). We were surprised to see the heptamer on SDS-PAGE gels after purification (Figure 
II.2.D), since most membrane proteins tend to fully dissociate even at very low concentrations of SDS; however oligomeric stability for harsh detergents have been observed for other membrane proteins like glycophorin A (Lemmon et al., 1992) and KcsA (Cortes and Perozo, 1997). Sukharev has also reported that even without the crosslinker (DSS) MscS exhibits a small amount of dimer (Sukharev, 2002). We also found that a fraction of $\mathrm{MscS}$ dimer (along with the monomer) was always present on the electrophoresis gels, even on the harshest conditions.

Mechanosensitive channel function relies on their interaction with the membrane, making reconstituted systems a requisite for their study (Martinac et al., 1990; Perozo et al., 2002a; Perozo et al., 2002b). The ability to reconstitute MscS in liposomes allows experimental control of intra bilayer forces using a variety of lipid mixtures and conditions that have specific influence over the TM pressure profile and thus influence channel conformation. Here, we offer a reliable purification system along with the characterization of $\mathrm{MscS}$ in liposomes that will open the door to the application of multiple structural approaches. Our electrophysiological results, in E. coli polar lipids, are in agreement with previous reports regarding single channel conductance and inactivation (Akitake et al., 2005; Sotomayor et al., 2007; Vasquez and Perozo, 2004).

The determination of the topology and the three dimensional architecture of membrane proteins by spectroscopic approaches relies on the fact that proteins have to be monodisperse on the plane of the bilayer; otherwise the acquired information will be affected by expurious protein-protein contacts, especially on those segments that are at the periphery of the molecule. The results obtained from EPR and FRET experiments show that the lipid systems commonly used for electrophysiological measurements, such 
as DOPC or E. coli polar lipids cannot be used for MscS structural determination, since they promote MscS two-dimensional aggregation. Perhaps, MscS aggregation naturally occurs in $E$. coli membranes and it might be essential for its function; but more studies are required in order to determine this phenomenon. 
CHAPTER III: Three Dimensional Architecture of Membrane-Embedded MscS in the Closed Conformation

\section{III.1 ABSTRACT}

The mechanosensitive channel of small conductance (MscS) is part of a coordinated response to osmotic challenges in E. coli. MscS opens as a result of membrane tension changes, thereby releasing small solutes and effectively acting as an osmotic safety valve. Both, the functional state depicted by its crystal structure and its gating mechanism remain unclear. Here, we combine site-directed spin labeling, electron paramagnetic resonance (EPR) spectroscopy, and molecular dynamics simulations with novel energy restraints based on experimental EPR data to investigate the native transmembrane and periplasmic molecular architecture of closed $\mathrm{MscS}$ in a lipid bilayer. In the closed conformation, $\mathrm{MscS}$ shows a more compact transmembrane domain than in the crystal structure, characterized by a realignment of the transmembrane segments towards the normal of the membrane. The previously unresolved $\mathrm{NH}_{2}$-terminus forms a short helical hairpin capping the extracellular ends of TM1 and TM2 and in close interaction with the bilayer interface. The present three-dimensional model of membraneembedded MscS in the closed state represents a key step in determining the molecular mechanism of MscS gating. 


\section{III.2 INTRODUCTION}

Cells rely on a tight regulation of the balance between internal and external osmotic forces to survive. In bacteria and archaea, osmoregulation is achieved by accumulation and release of solutes through active transport systems and mechanosensitive (MS) channels, respectively (Hamill and Martinac, 2001; Poolman et al., 2002; Wood, 1999). MS channels act as indirect osmosensors in the emergency response to hypo-osmotic challenges. When water enters the cell, the turgor pressure increases and the membrane expands reducing its lateral pressure, which leads to MS channels opening. The subsequent release of solutes through the open MS channels alleviates turgor pressure and prevents cell lysis (Levina et al., 1999). The E. coli inner membrane has several MS channels involved in this complex response (Berrier et al., 1996; Martinac et al., 1987). These channels have been classified, according to their single-channel conductance and the tension required to activate them, as MS channel of Large, Small, and Mini conductance, or MscL, MscS/MscK, and MscM, respectively (Levina et al., 1999).

MscL and MscS x-ray crystal structures (Bass et al., 2002; Chang et al., 1998) have provided an important framework to understand the gating of MS channels and interpret experimental results. However, while both $\mathrm{MscL}$ and $\mathrm{MscS}$ seem to respond to similar bilayer deformations and the MscL structure has lead to explicit modeling of its open and closed conformations (Anishkin et al., 2003; Gullingsrud and Schulten, 2003; Perozo et al., 2001, 2002b; Sukharev et al., 2001a), the MscS gating mechanism and the functional state depicted by its crystal structure remain unclear. The MscS crystal is arranged as a homoheptamer with three transmembrane (TM) segments per subunit 
(TM1, TM2, and TM3A-B) and a large cytoplasmic domain featuring seven side "windows" and a narrow opening at the most distal region from the membrane (Figures III.1.A, B). The first 26 residues of the $\mathrm{NH}_{2}$-terminus, a region that appears to play a role in gating and stability, are not resolved in the crystal structure. As the narrowest point along the permeation path is $\sim 7 \AA$ in diameter, it was originally suggested that this conformation represented the open state of MscS (Bass et al., 2002), its dimensions putatively supporting its $1 \mathrm{nS}$ single channel conductance and slightly selectivity towards anions. Molecular dynamics (MD) and continuum simulations have systematically shown that the crystal conformation exhibits low conductance, rectification at high voltages, and high anionic selectivity (Anishkin and Sukharev, 2004; Sotomayor and Schulten, 2004; Sotomayor et al., 2006; Sotomayor et al., 2007; Spronk et al., 2006; Vora et al., 2006). Hence, while the current consensus is that the $\mathrm{MscS}$ crystal conformation is unlikely to represent the fully open state, its precise functional assignment remains to be established. It is then clear that to determine the conformations of the closed and open states and thereby understand the mechano-gating process underlying MscS function in vivo, additional structural and dynamical information of $\mathrm{MscS}$ in a native-like membrane is required.

Electron paramagnetic resonance (EPR) spectroscopy is a powerful tool for structural analysis of soluble and membrane proteins in native-like conditions. This technique is particularly useful for judging crystal structures obtained in non-native environments (Cuello et al., 2004; McHaourab et al., 1996; Perozo et al., 1998; Perozo et al., 2001). Site-directed spin labeling (SDSL) and EPR spectroscopy gives three types of structural information: spin-label motion, solvent accessibility, and distance 
measurements (Columbus et al., 2001; McHaourab et al., 1996). It is therefore possible to determine whether a spin-labeled position is exposed to the solvent or the membrane, or buried within the protein. Altogether, the EPR data in the context of a lipid bilayer offers an extensive collection of accessibilities constraints that considerably decreases the number of possible folds, in order to derive along with computational methods threedimensional models of proteins, in their native environments and in different conformational states (Cortes et al., 2001; Koteiche and McHaourab, 1999; Perozo et al., 1999).

Here, we have analyzed the structural dynamic properties of membranereconstituted $\mathrm{MscS}$ in the closed state conformation, as determined by SDSL, EPR spectroscopy and computational modeling. The experimental data was used to refine the crystal structure under known physiological conditions, and to construct a model of the closed conformation of MscS that includes the previously unresolved $\mathrm{NH}_{2}$-terminus. The new model shows significant differences regarding the position of the TM1-TM2 hairpin, and the arrangement of TM2 with respect to TM3. We find that the $\mathrm{NH}_{2}$-terminus is a mixed helical hairpin, placed at the periplasmic interface of the lipid bilayer, away from the channel central axis of symmetry. Our experimental data suggest that the MscS closed state is in a different and a more compact conformation than the one trapped in the crystal structure. 
A

Cysteine Mutants
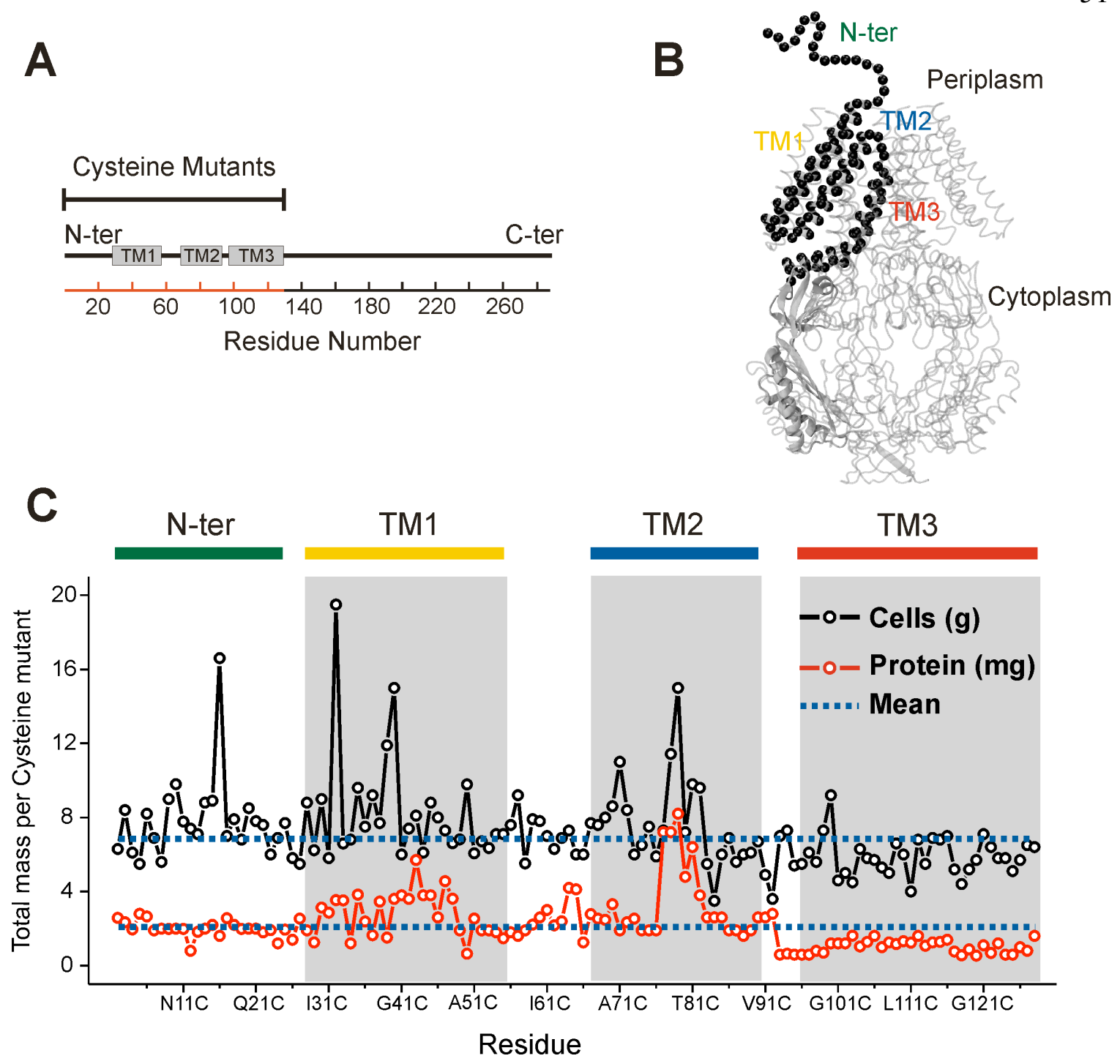

Figure III.1. MscS architecture and cysteine mutants expression A) Linear representation of MscS topology. The TM helices (as suggested from the crystal structure) of the MscS monomer (TM1, TM2, and TM3) are represented by rectangles. The scale corresponds to the amino acid residue numbering. The red line denotes the residues that were mutated to cysteine. B) Single MscS subunit showing amino acid residues (black spheres) probed through site directed spin labeling of cysteines. A single MscS monomer is represented as part of the heptamer according to the crystal structure of the channel. The $\mathrm{NH}_{2}$-terminus segment (26 residues) is represented by a schematic line. C) Expression of cysteine mutants. The total amount of cell and protein obtained is shown for each residue after expressing single cysteine mutants in vector pET28 (containing MscS with His6 epitope at the $\mathrm{NH}_{2}$-termini), in E. coli Rosetta $^{\mathrm{TM}}$ cells. Grayed areas represent the membrane embedded areas derived from the MscS crystal structure. 


\section{III.3 EXPERIMENTAL PROCEDURES}

\section{III.3.1 Mutagenesis, Expression, Spin-Labeling, and Reconstitution of MscS}

Cysteine mutants were generated for residues 2-128 in $\mathrm{MscS}$, covering the $\mathrm{NH}_{2}$ terminus, TM1, TM2, and TM3. Mutagenesis was performed using the QuickChange ${ }^{\mathrm{TM}}$ Site-Directed Mutagenesis Kit (Stratagene). Mutant channels were expressed, purified, labeled and reconstituted as previously described (Vasquez et al., 2007a) using a pET28 vector containing $\mathrm{MscS}$ with His6 epitope at the $\mathrm{NH}_{2}$-terminus and a $\mathrm{pQE70}$ vector with His6 epitope at the COOH-terminus, for the study of the TM segments and the $\mathrm{NH}_{2}$ terminus, respectively. MscS-pET28 was used to express the channel in E. coli Roseta $^{\mathrm{TM}}$ and MscS-pQE70 was used for expression in E. coli M15. The samples were reconstituted at a 750:1 lipid/channel (DOPC:POPG, 6:1) molar ratio by dilution in PBS in the presence of Bio-beads. To determine the interfacial residues a mixture of DOPC:POPG:DOGS-NTA[Ni(II)]lipids, in a molar ratio of 6:2:3, respectively, was used.

\section{III.3.2 Pegylation}

After purification under reducing conditions, cysteine mutants at positions S9C, G12C, and Y27C were fully-labeled (10:1, label/monomer) and under-labeled (1:10) with PEG-maleimide (Lu and Deutsch, 2001, 2005). The changes in MscS monomer relative molecular weight were analyzed on SDS-PAGE gels.

\section{III.3.3 Liposome and Spheroplast Patch Clamp}

Liposome single-channel currents were obtained in the inside-out configuration under symmetrical conditions $\left(200 \mathrm{mM} \mathrm{KCl}, 90 \mathrm{mM} \mathrm{MgCl}, 10 \mathrm{mM} \mathrm{CaCl}_{2}\right.$, and $5 \mathrm{mM}$ HEPES), at $\mathrm{pH}$ 6. Macroscopic currents were recorded by patch-clamping giant spheroplasts as described elsewhere (Martinac et al., 1987). Glass pipettes were pulled 
and fire-polished to resistances between 2 and $2.5 \mathrm{M} \Omega$. Negative pressure on the patch was established by applying suction through a high-speed pressure clamp (ALA Scientific Instruments Inc.). Single-channel currents were recorded with a DAGAN 3900 patch-clamp amplifier, and currents were sampled at $10 \mathrm{kHz}$ with an analog filter set to 2 kHz (Sotomayor et al., 2007; Vasquez et al., 2007a).

\section{III.3.4 EPR Spectroscopy and Data Analysis}

All EPR data were obtained at room temperature. X-band CW EPR spectra were performed as previously described (Perozo et al., 1998). Spectra were obtained in a Bruker EMX spectrometer fitted with a loop gap resonator under the following conditions: $2 \mathrm{~mW}$ incident power, $100 \mathrm{kHz}$ modulation frequency and $1 \mathrm{G}$ modulation. For DOGS-NTA[Ni(II)]lipids experiments a dielectric resonator was used. The accessibility parameters were quantified as previously reported (Farahbakhsh et al., 1992; Gross and Hubbell, 2002; Perozo et al., 1998). Briefly, collision frequency is directly proportional to the accessibility of the paramagnetic reagent to the nitroxide, and is defined as

$$
\Pi=\left[\Delta \mathrm{P}_{1 / 2}(\mathrm{X}) / \mathrm{P}_{1 / 2}(\mathrm{DPPH})\right] \times\left[\Delta \mathrm{H}_{\mathrm{o}}(\mathrm{DPPH}) / \Delta \mathrm{H}_{\mathrm{o}}\right]
$$

where $\mathrm{P}_{1 / 2}(\mathrm{DPPH})$ is the microwave power that saturated the signal relative to that without saturation for a 2,2-diphenyl-1-picrylhydrazyl crystal (molecule with a single unpaired electron), $\Delta \mathrm{P}_{1 / 2}(\mathrm{X})$ is the difference in $\mathrm{P}_{1 / 2}$ of a given sample exposed to $\mathrm{O}_{2}$ or NiEdda and $\mathrm{N}_{2} . \Delta \mathrm{H}_{\mathrm{o}}$ is an estimate of probe mobility derived from the spectral lineshape, determined by the degree of averaging of the anisotropic $g$ value (McHaourab and Perozo, 2002). 


\section{III.3.5 Functional Assays}

Downshock assays were performed as described earlier (Batiza et al., 2002), using the E. coli strain MJF465 (kindly donated by Ian Booth (Levina et al., 1999). Method modifications and details were provided earlier (Vasquez et al., 2007a). In brief, cultures growing in $\mathrm{LB}$ and $500 \mathrm{mM} \mathrm{NaCl}$ were induced at an $\mathrm{OD}_{600}$ of 0.6 with $1 \mathrm{mM}$ IPTG; after being induced for $1 \mathrm{~h}$, the cells were adjusted to an $\mathrm{OD}_{600}$ of 1.0, pelleted down, and then resuspended in $50 \mu \mathrm{L}$ of fresh $\mathrm{LB}$ and $500 \mathrm{mM} \mathrm{NaCl}$. The downshock was made by diluting the cells 1:40 in various shock media ranging from 0 to $500 \mathrm{mM} \mathrm{NaCl}(\mathrm{pH} 7)$. After $5 \mathrm{~min}$, the cells were serially diluted and $5 \mu \mathrm{L}$ aliquots were plated on LB agar. Plated cells were grown overnight, and the colonies were counted and normalized against the $\mathrm{OD}_{600}$ (measured right before the downshock).

\section{III.3.6 MscS N-terminal Model and Pseudo-Atom driven Solvent} Accessibility Refinement

The pseudo-atom driven solvent accessibility refinement was done in collaboration with Marcos Sotomayor, from Klaus Schulten Laboratory (University of Illinois at Urbana-Champaign), and Benoît Roux from The University of Chicago.

An initial, symmetrized model of $\mathrm{MscS}$ was built from monomer $\mathrm{C}$ of the refined MscS crystal structure (pdb code 2AOU) (Steinbacher et al., 2007) including residues 1 to 178 . The model included a conformation of the $\mathrm{NH}_{2}$-terminus obtained using the software Rosetta (Bonneau et al., 2002; Simons et al., 1997) and applying energy minimization in vacuum (Figure III.12.A). In addition to protein atoms, two EPR virtual particles were harmonically attached to each $\mathrm{C}-\alpha$ atom of the protein, following the method described by (Sompornpisut et al., 2007)(Sompornpisut et al, 2007; unpublished 
data). The first EPR particle, labeled PROT, was attached on top of the C- $\alpha$ atom, while the second EPR particle, representing a spin label probe, was attached through a bond with a resting length of $6 \AA$ (Figure III.12.B).

The water or lipid exposed pseudo-spin probes were assigned attractive or repulsive interactions to additional $\mathrm{O}_{2}$ and $\mathrm{NiEdda}$ virtual environment particles, distributed so as to mimic the membrane location, while the buried pseudo-spins were allowed to artificially overlap with the backbone core (PROT particles). Assignment of interactions was performed based on EPR accessibilities as indicated on the text.

Structural refinement simulations were performed using the CHARMM program version c32a2 (Brooks et al., 1983) modified to implement the novel methodology. The extended atom PARAM19 force field was modified to reduce the effect of charged residues in vacuum according to the scheme of Lazaridis and Karplus (Lazaridis and Karplus, 1999). Harmonic restraints were applied to keep $\mathrm{O}_{2}$ particles away from the MscS pore, to keep residues 5, 23, 53, 57, 60, and 127 at the membrane-water interface, and to force side-chains of residues 23 and 27 to face away from the center of the channel. In addition, RMSD constraints were applied (to maintain the initial secondary structure) to residues 10 to 14,20 to 26,29 to 57,68 to 91,96 to 113,114 to 128 , and 129 to 178. Multiple minimization and equilibration cycles were performed. After ten million steps ( $1 \mathrm{fs} /$ time step), the $\mathrm{NH}_{2}$-terminus was rotated and further equilibration was carried out.

\section{III.3.7 Molecular Dynamics Simulations}

All the Molecular Dynamics Simulations, in this chapter, and its respective analysis were done in collaboration with Marcos Sotomayor, from Klaus Schulten 
Laboratory (University of Illinois at Urbana-Champaign).

Two systems were prepared for simulation. The first system contained the recently released structure of $\mathrm{MscS}$ at $3.7 \AA$ resolution (residues 27 to 280). The second system contained the EPR-refined model (residues 1 to 178). In both cases the protein was embedded in a fully hydrated membrane (POPC) environment, as described earlier (Sotomayor and Schulten, 2004). Ions were placed randomly to reach concentrations of $200 \mathrm{mM} \mathrm{KCl}, 90 \mathrm{mM} \mathrm{MgCl}$, and $10 \mathrm{mM} \mathrm{CaCl}_{2}$ for each system. The resulting systems encompassed 264,000 and 210,000 atoms, respectively.

Simulations were performed using NAMD2.6 (Phillips et al., 2005), the CHARMM27 force-field for lipids and proteins (MacKerell et al., 1998), the CMAP correction (Mackerell et al., 2004), the TIP3 model for water, and a uniform $1 \mathrm{fs}$ time step. Other simulation details were described earlier (Sotomayor and Schulten, 2004). Both systems mentioned above were subject to 4 ns of equilibration in which lipid tails were melted, water was moved out from the membrane-protein interface, and initial constraints on protein side chains and backbone were stepwise released. Equilibration achieved a good packing of lipids around the protein. For each system six nanoseconds of free dynamics were performed after equilibration. 


\section{III.4 RESULTS}

\section{III.4.1 Expression and Purification of MscS Cysteine Mutants}

Individual residues along the complete $\mathrm{TM}$ domain of $\mathrm{MscS}$ were sequentially mutated to cysteine and subsequently spin labeled (WT MscS is cysteine less). The targeted regions are highlighted in Figure III.1.A along a schematic representation of the MscS sequence, where individual rectangles represent the approximate length of the $\alpha$ helical TM segments according to the crystal structure (Bass et al., 2002). The mutated residues (2 to 128) are also shown in Figure III.1.B as black spheres on a ribbon diagram of the MscS monomer, and over a schematic line in the structurally-undetermined $\mathrm{NH}_{2}$ terminus.

All MscS cysteine mutants were expressed, purified in reducing conditions, labeled, and reconstituted into pre-formed DOPC:POPG liposomes, as previously described (Vasquez et al., 2007a). None of the mutations were associated with significant gain of function (GOF) phenotypes that would result in lethal leakage of solutes during cell growth, since the cultures optical density did not decrease upon induction with IPTG. Instead, some cysteine substitutions at conserved sites (Bass et al., 2002) promoted cell growth (W16C at the $\mathrm{NH}_{2}$-terminus; V32C and V40C at TM1; and A79C at TM2).

Mutant expression yield was in general below WT MscS ( $4 \mathrm{mg}$, Figure III.1.C), and even lower for mutations at the $\mathrm{NH}_{2}$-terminus and the TM3 helix. Despite these differences, heptameric oligomerization was always achieved. This suggests that mutant purification under reducing conditions did not affect the overall architecture of the channel, i.e. not compromising the heptameric structure in the formation of spontaneous disulfide bridges, as suggested by gel filtration analysis and SDS-PAGE gels (Figure 
III.2). Mutations on the narrowest part of the MscS permeation pathway (positions 93101, 107 and 115) promoted some dissociation of the heptamer into an unfolded channel, which represented a small fraction of the total protein (Vasquez et al., 2007a). For these particular TM3 mutants, EPR spectroscopy was performed on the fraction that corresponded to the heptamer, as determined from gel filtration analysis (Figure III.2). 


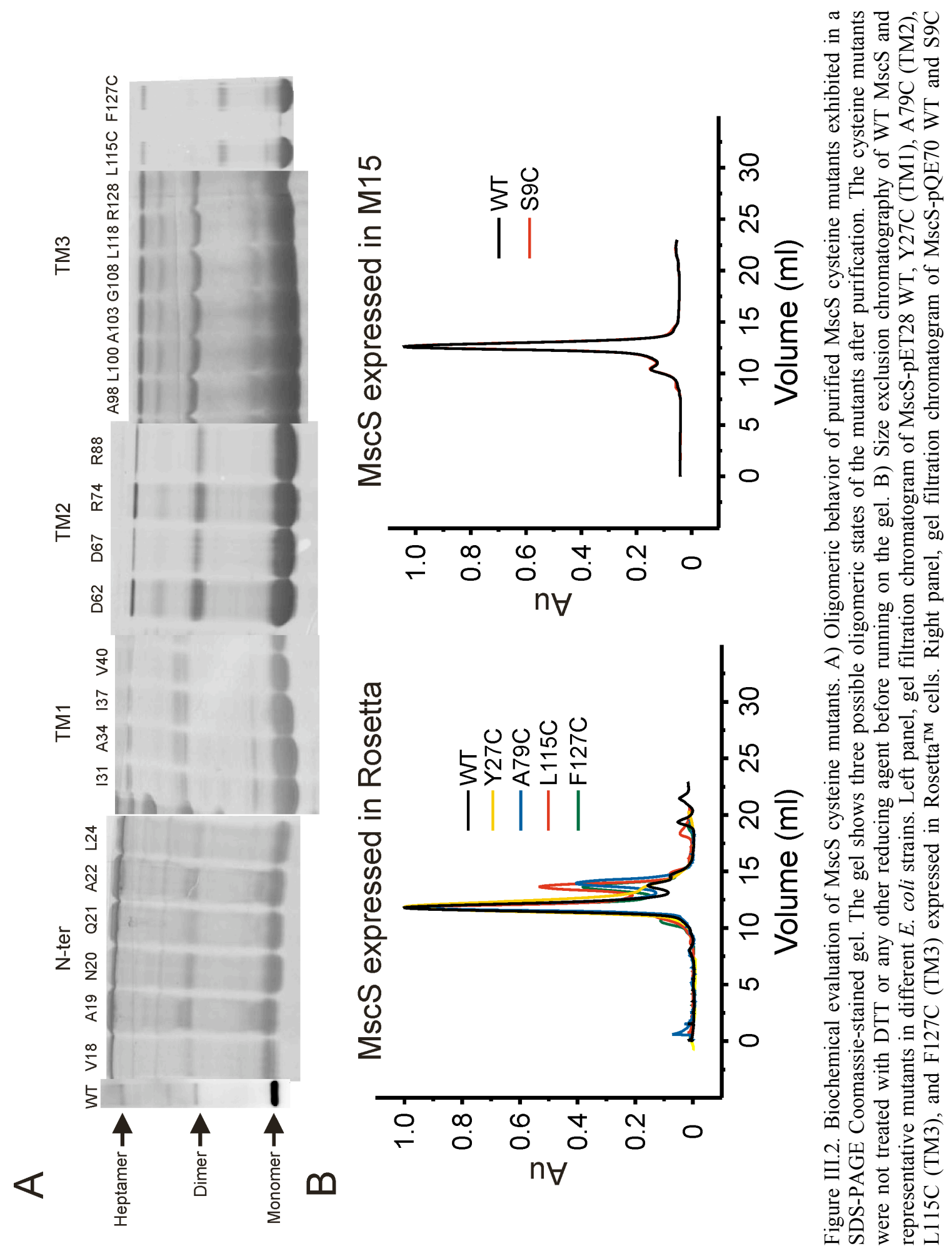




\section{III.4.2 Environmental Parameters of MscS TM segments}

Spin labeled mutants were subject to CW-EPR spectroscopic measurements on liposomes, under zero transbilayer pressure and in absence of a TM voltage (conditions where $\mathrm{MscS}$ is known to be closed, Figure III.3). It is expected that under these conditions the spectroscopic signal will come overwhelmingly from $\mathrm{MscS}$ in its closed state, with negligible contributions from other conformations. Nitroxide mobility and accessibility to $\mathrm{O}_{2}$ and NiEdda were measured for each MscS spin labeled mutant, and accessibility to the lipid Ni(II) chelate (DOGS-NTA) was evaluated in selected mutants at the $\mathrm{NH}_{2}$-terminus, TM1-TM2 loop, and at the end of the TM3B. It must be noted, however, that we have been unable to estimate inter-subunit distances or proximities in the present data set. This is likely due to under-labeling of samples caused by the presence of genomic MscS monomers (Figure III.4). The high natural abundance of these cysteine-less monomers in the population of heptameric channels sharply reduce the probability of finding spin-spin coupling between adjacent subunits, and limits our ability to determine inter-subunit distances. However, this fact does not affect the quality and usefulness of the environmental data from under-labeled channels.

A representative set of EPR spectra obtained for MscS' TM1 is shown in Figure III.5.A. The fully processed EPR environmental data set for the $\mathrm{NH}_{2}$-terminus and TM segments is shown in Figure III.5.B. Mobility and $\mathrm{O}_{2}$ accessibility parameters indicate a clear trend for the dynamics and membrane exposure of the TM domain. Excluding the first 26 residues of the $\mathrm{NH}_{2}$-terminus (see analysis below), residues located towards the $\mathrm{NH}_{2}$-terminal region of TM segments, periplasmic face of TM1 and cytoplasmic face of TM2, are more mobile and more exposed to $\mathrm{O}_{2}$ than those at the COOH-terminal. 
Average mobility and $\mathrm{O}_{2}$ accessibility (top and middle panels in Figure III.5.B, respectively) were found to be higher in TM1 and at the $\mathrm{NH}_{2}$-terminal segment of TM2 than those obtained for TM3. On the other hand, the EPR data suggests that the environment of TM3 helices is sterically restricted, as reflected by a lower $\mathrm{O}_{2}$ exposure and lower overall dynamics than seen for the remaining TM helices (see box plots in Figure III.11.B). This behavior is expected for a segment that lines the conduction pathway of an ion channel in its closed conformation. A patch of the TM3 segment (positions 104 to 112 ) exhibits higher mobility and $\mathrm{O}_{2}$ accessibility than the TM3 average (dotted box on middle panel of Figure III.5.B). This can be interpreted to support the hypothesis that the ion flow may be blocked by the hydrophobicity of the surface that contours the pore in the closed state (Anishkin and Sukharev, 2004).

Although the MscS crystal structure was obtained in detergent, the EPR-derived environmental data mapped onto its surface (Figures III.5.C and III.16.A) reveals interesting correlations between the two types of structural data. First, probe dynamics and exposure to aqueous environment in the TM1-TM2 connecting loop (upper and bottom panel in Figure III.5.B-C) appears to be inconsistent with the boundary between TM1 and TM2 segments predicted by the crystal structure (Bass et al., 2002). Positions exposed to NiEdda at the TM1-TM2 hairpin appear to be in the non-polar region of the membrane (bottom panel in Figure III.5.C), while highly mobile and $\mathrm{O}_{2}$ exposed residues tend to locate at the cytoplasmic region of TM2 (upper and middle panel in Figure III.5.C, and left panel in Figure III.16.A). As NiEdda-accessible residues seem to cluster toward the COOH-terminal end of TM1 (bottom of Figure III.5.C and middle panel in Figure III.16.A), we would suggest that to match the membrane boundaries to the 
experimental data, these should shift about two turns of the helix towards the extracellular face of the channel in relation to putative boundaries based on the crystal structure.

Residue solvent accessibilities obtained using hard-sphere methods on the crystal structure were used to calculate TM1 and TM2 environmental moments, which were compared with the $\mathrm{O}_{2}$ accessibilities $\left(\mathrm{\Pi O}_{2}\right)$ resultant moment obtained from EPR data (Figure III.6). The position of the resultant vectors agrees with the TM1 being exposed to two environments (lipidic and proteinaceous), and the TM2 COOH-terminal region facing a proteinaceous homogenous environment. For TM3, environmental moments could not be calculated, since overall mobility and accessibilities were quite low compared to those of the other TM segments. Within this segment, however, residues G104C-SL to Q112C-SL exhibited higher $\mathrm{O}_{2}$ accessibility than the TM3 average. Although the overall topology suggested by EPR environmental parameters in a membrane environment is consistent with the one depicted by the available MscS crystal structure, the data suggests that the closed conformation of MscS has to feature a TM2 helix that is packed against TM3. This suggests a more compact arrangement of the TM helices relative to each other and shifted downwards in relation to the plane of the bilayer. 

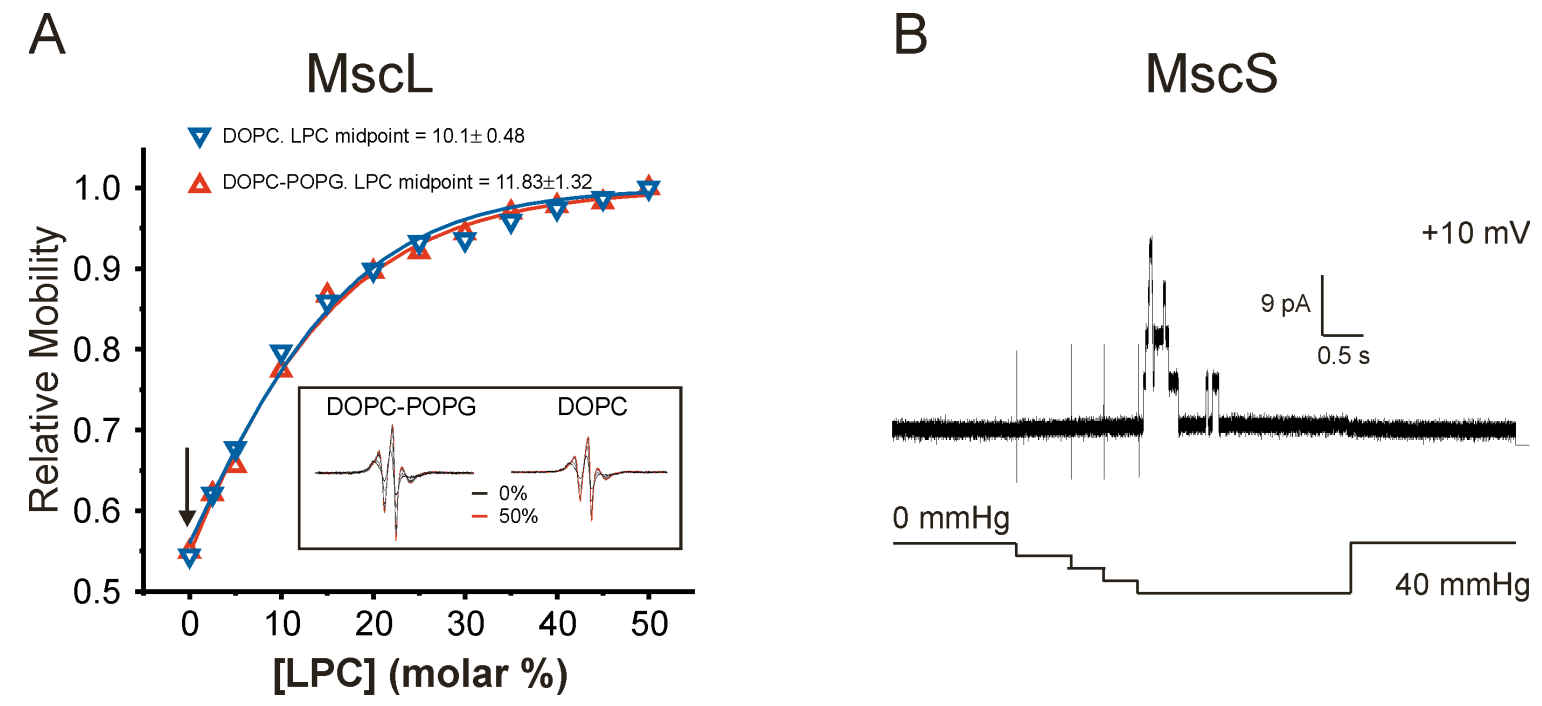

Figure III.3. MscS closed state characterization. A) Concentration dependence of the LPC conformational changes in MscL V23C-SL monitored from the effects on probe dynamics, in DOPC:POPG and DOPC liposomes. The arrow points the condition where MscL is in the closed state. We evaluated MscL activation curve in DOPC (Perozo et al., 2002a) and DOPC:POPG. The mid-activation point and the steepness of MscL opening curve was not affected by the composition of the mixed bilayer used in $\mathrm{MscS}$ experiments. B) Single-channel record from purified MscS reconstituted in DOPC:POPG lipids. Channel activity was only elicited by applying negative transbilayer pressure under patch-clamp conditions. 

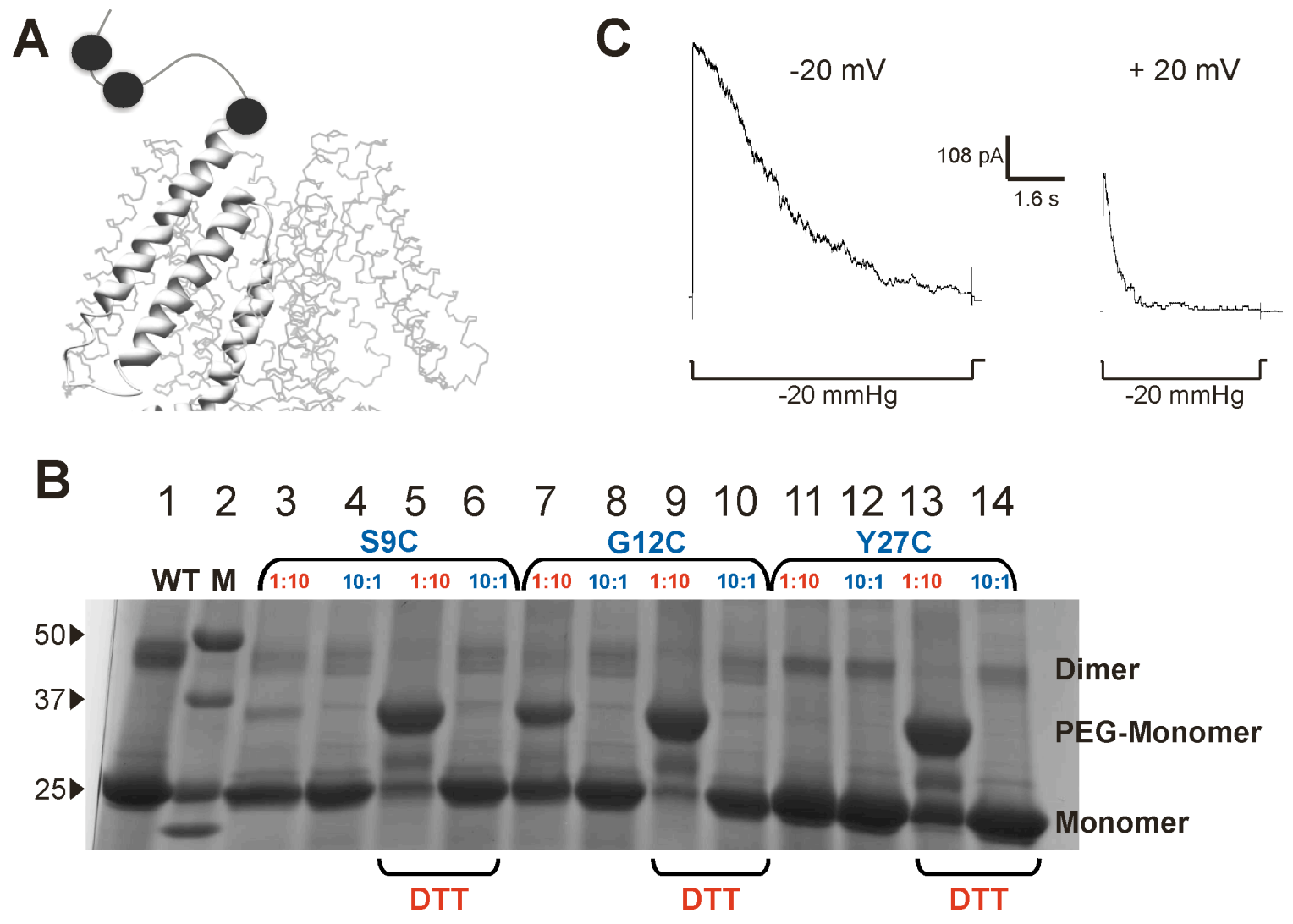

Figure III.4. Labeling efficiency as determined by cysteine pegylation ( $\mathrm{Lu}$ and Deutsch, 2001, 2005). A) Ribbon diagram showing individual residues with low (S9C) and high accessibilities to NiEdda (G12C and Y27C) as determined by EPR, that were labeled with PEG-maleimide ( $5 \mathrm{kDa})$. B) SDS-PAGE gel stained with Coomassie Blue of mutants labeled with PEG-maleimide. Lane 1: WT MscS, Lane 2: molecular weight marker, Lanes 3-6: S9C, Lane 3: fully-labeled (1 monomer : 10 PEGmaleimides). Lane 4: under-labeled (10 monomers : 1 PEG-maleimide). Lane 5: DTT prior to fully-labeling. Lane 6: DTT prior under-labeling. Lanes 7-10: G12C, Lane 7: fully-labeled. Lane 8: under-labeled. Lane 9: DTT prior to fully-labeling. Lane 10: DTT prior under-labeling. Lanes 11-14: Y27C, Lane 11: fully-labeled. Lane 12: under-labeled. Lane 13: DTT prior to fully-labeling. Lane 14: DTT prior underlabeling. C) Macroscopic currents were obtained under constant pressure at $+20 \mathrm{mV}$ and $-20 \mathrm{mV}$, at least 66 channels were on the patch. 

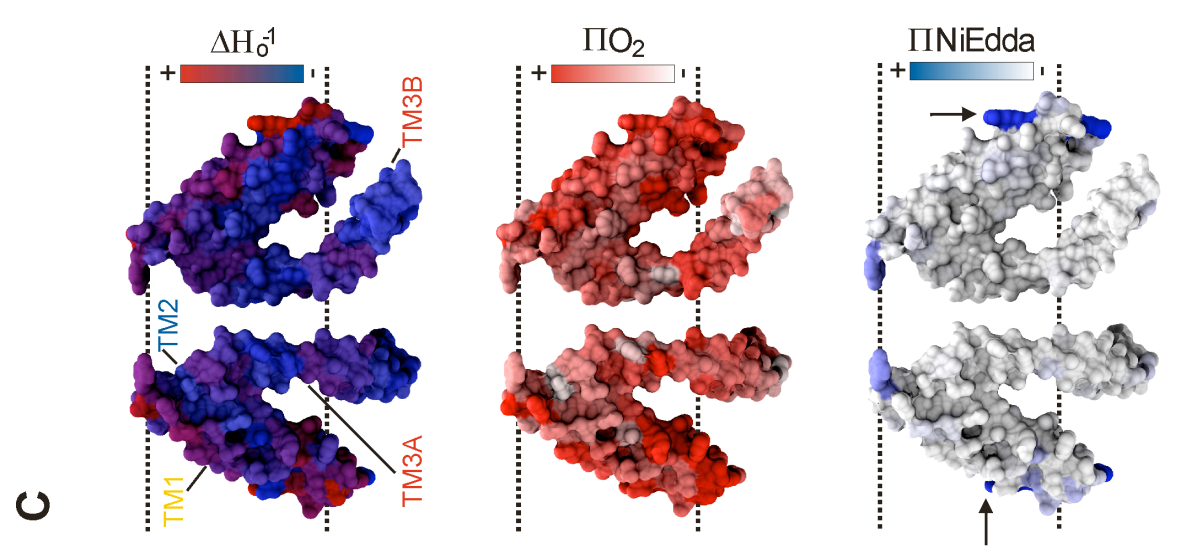

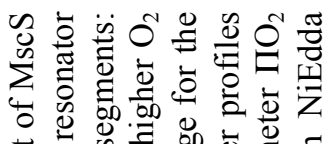

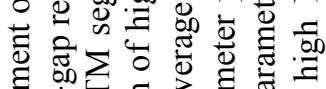

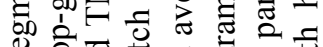

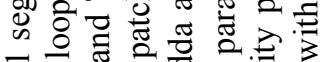

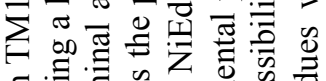

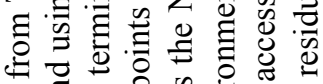

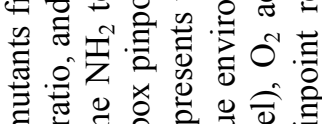

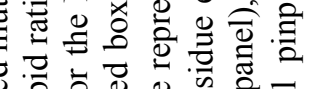

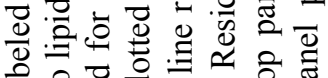

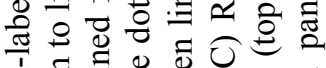
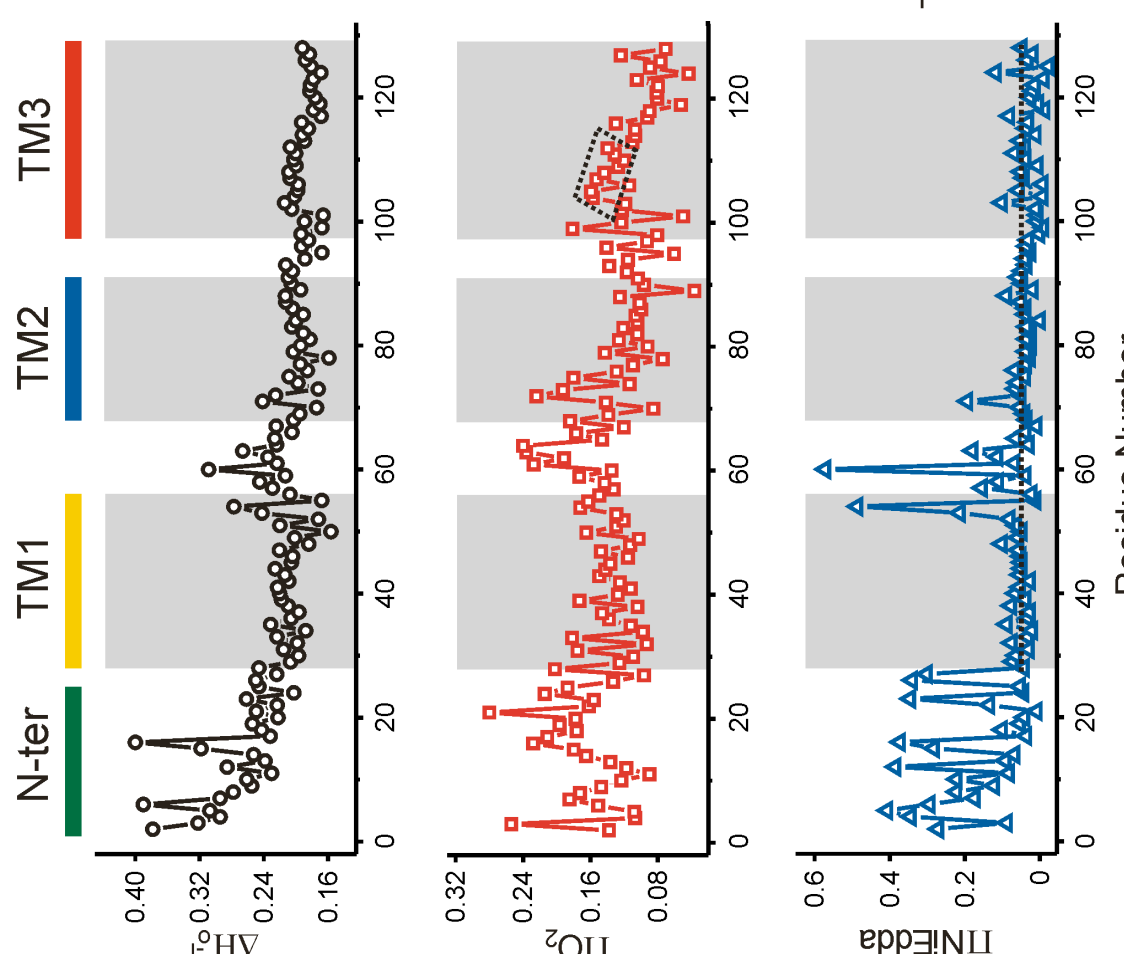

品寻芯冚

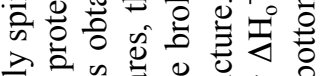

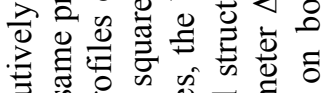

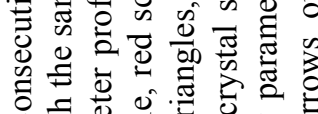

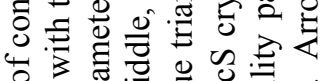

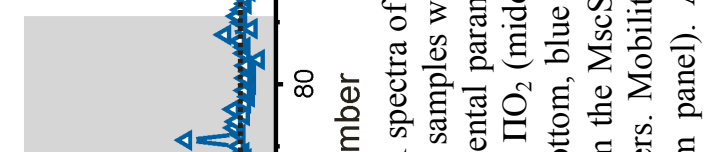

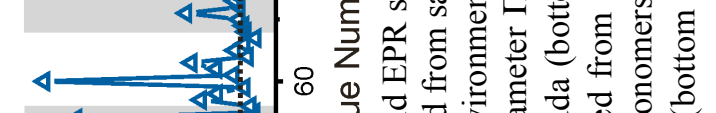

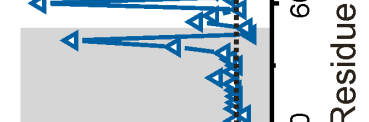
ज च

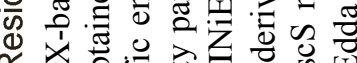
$q \propto$

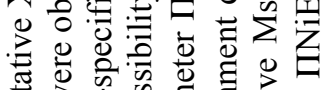

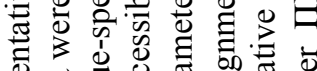

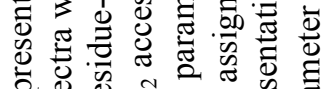

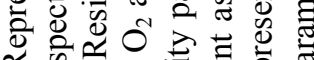

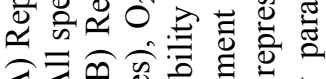

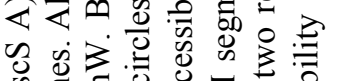

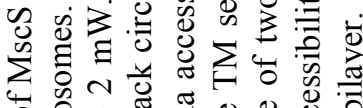
4.

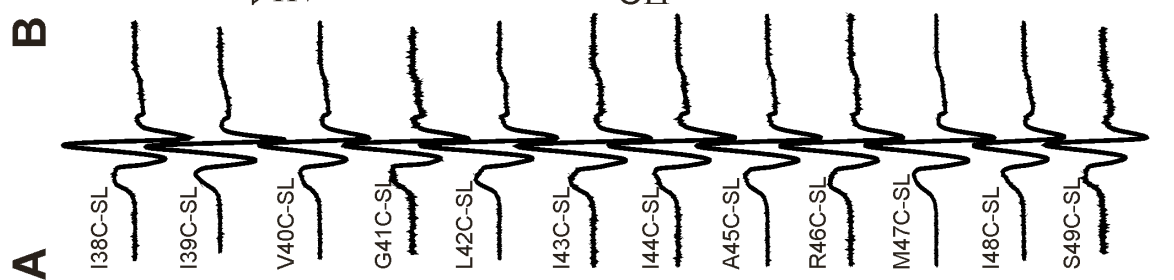

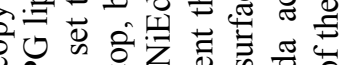
论 원 क्षे⿺辶寸

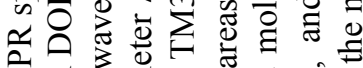

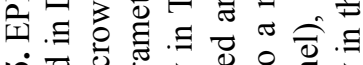

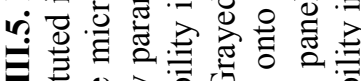

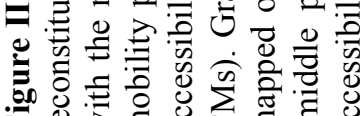




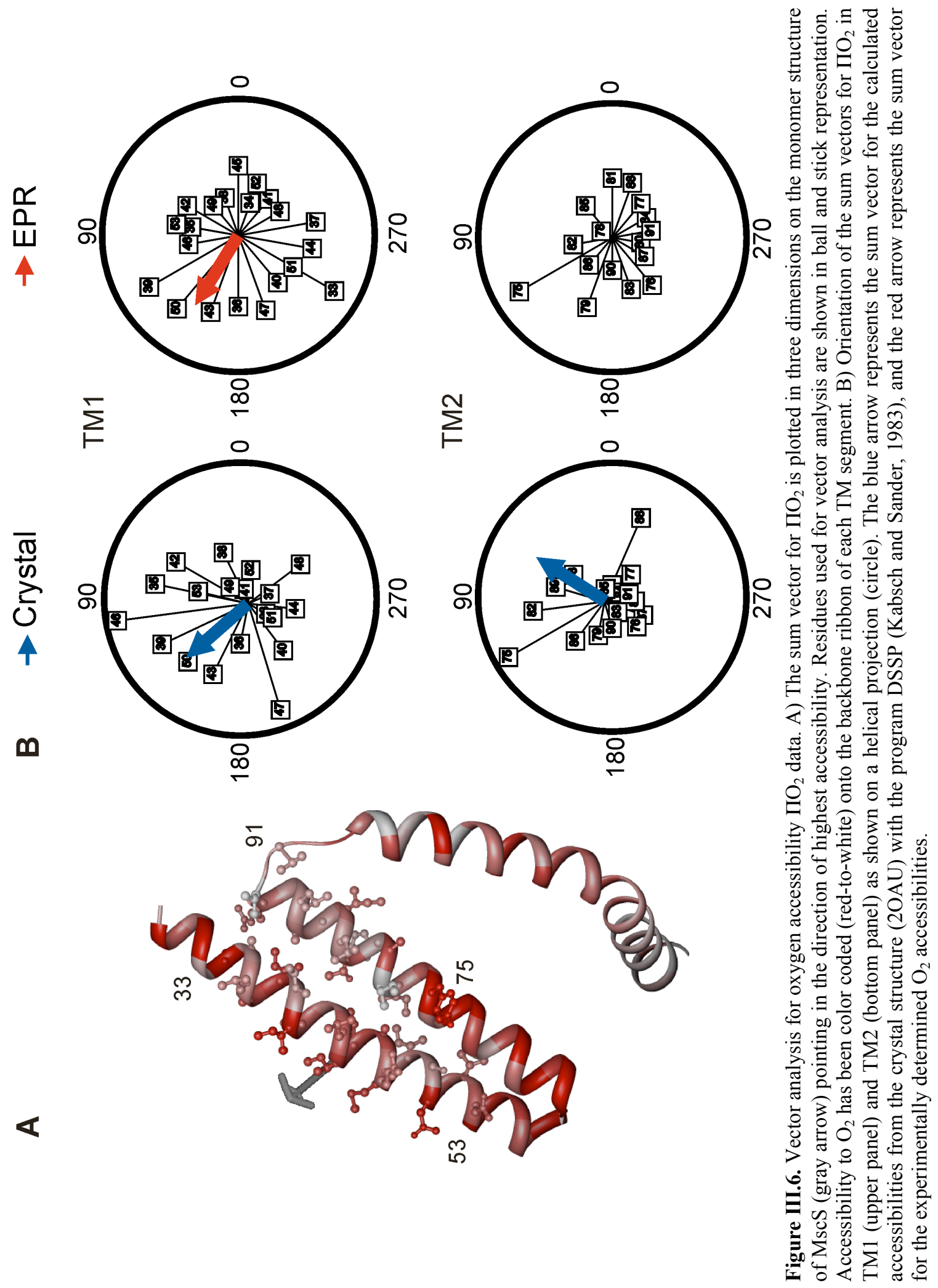


To assist us in predicting the position of MscS TM domain with respect to the membrane, we used DOGS-NTA[Ni(II)]lipids as a probe of residue interfacial positioning. These synthetic lipids confine the Ni(II) relaxing agent within a region of 14 $\AA$ at the membrane aqueous interface (14 $\AA$ being the maximum distance in the lipid extended configuration, Figure III.7.A) (Gross and Hubbell, 2002). Figure III.7.B and C shows the DOGS-NTA collisional frequency data obtained for positions at the end of TM1 and the beginning of TM2. We used two contrasting residues in the middle of the bilayer as experimental controls in order to set the background collisional levels, I39CSL is one of the most $\mathrm{O}_{2}$ exposed positions in the core of the membrane while G41C-SL is one of the most buried ones (see red arrows on Figure III.7.B). According to these environmental parameters, the TM1-TM2 loop (positions 50-61) and positions M126CSL, F127C-SL, and R128C-SL are at the membrane-aqueous interface, with positions N53C-SL, R54C-SL, K60C-SL, and A63C-SL largely exposed to the aqueous compound NiEdda. 


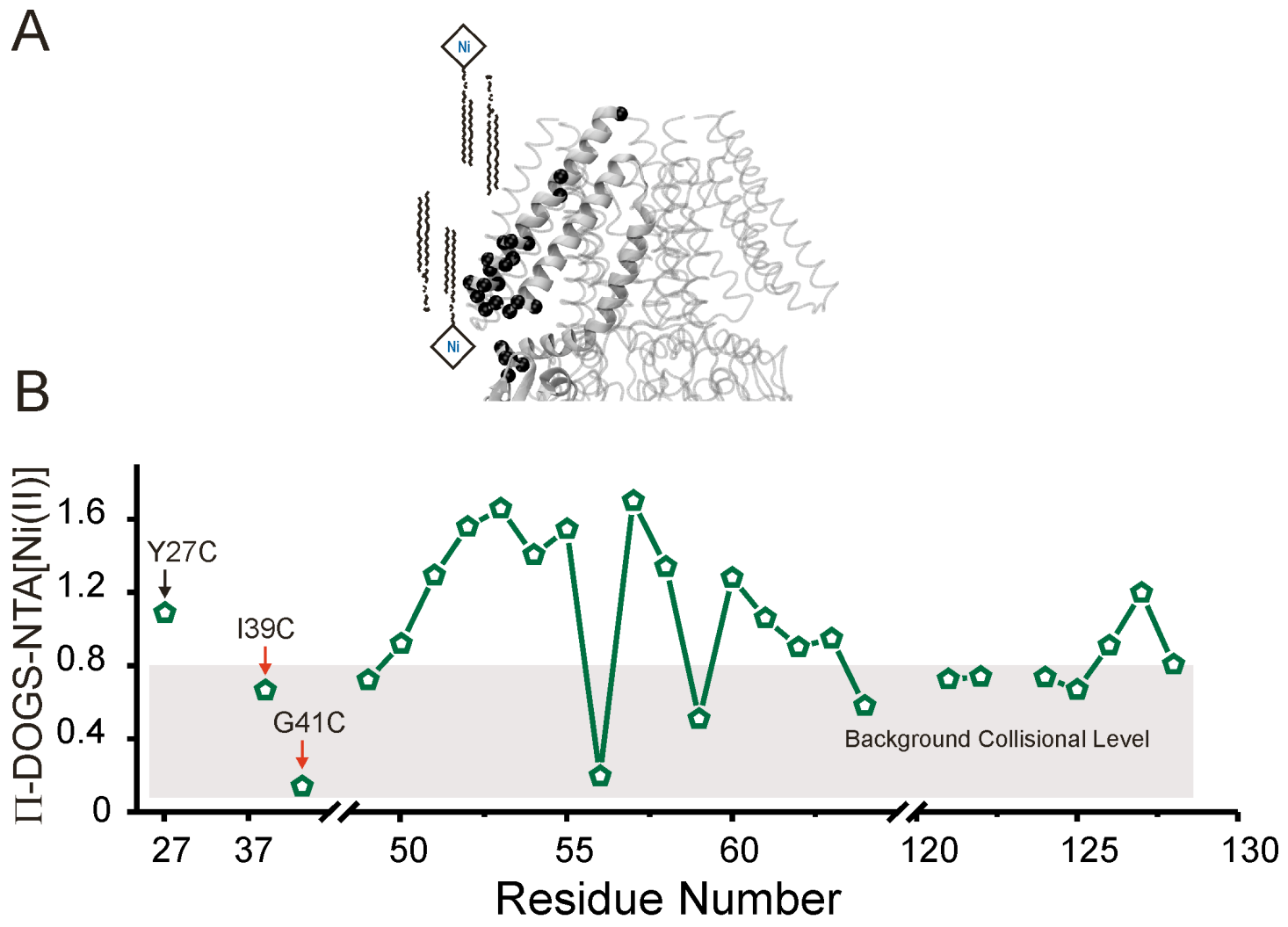

C

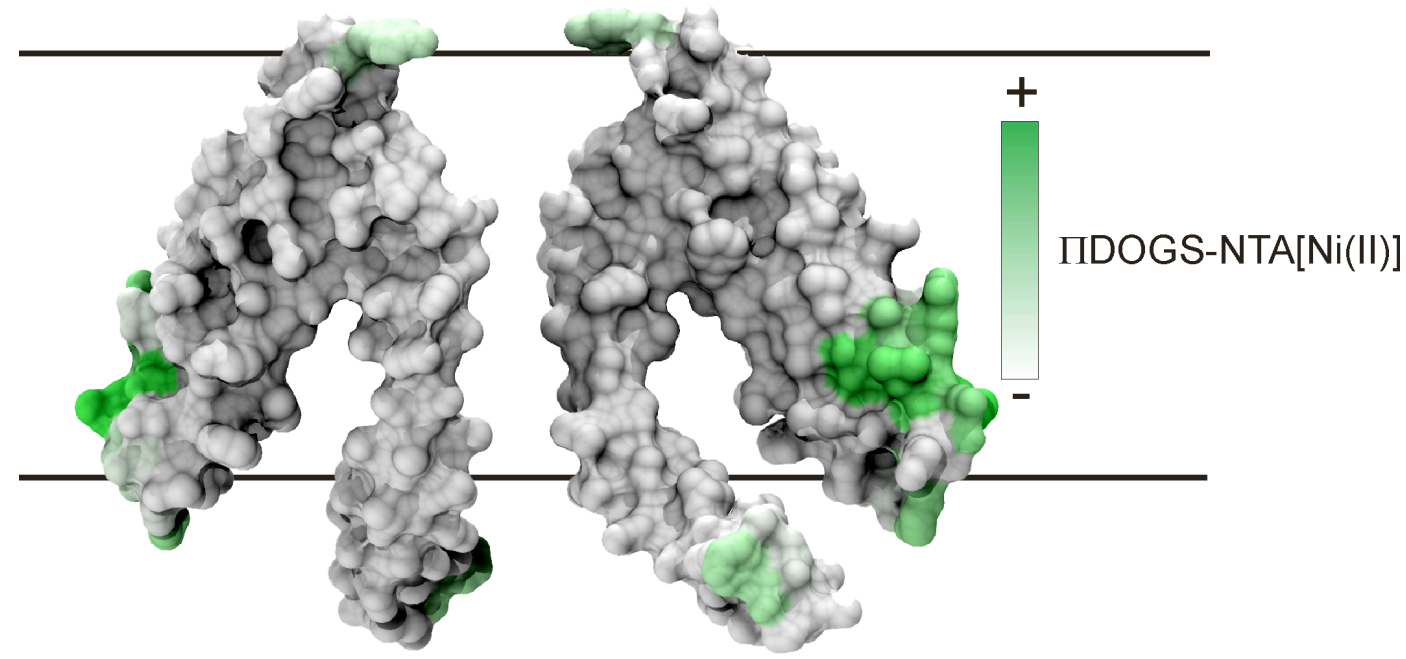

Figure III.7. Identification of MscS interfacial residues with DOGS-NTA[Ni(II)]lipids. A) Ribbon diagram of the TM segments of the MscS crystal structure (a single MscS monomer is highlighted) embedded in a schematic membrane consisting of DOPC:POPG and DOGS-NTA[Ni(II)]lipids (few lipid molecules are shown for clarity). Black spheres show the location of cysteine mutants that were used to obtain the data. B) Residue environmental parameter profile derived from Y27C (black arrow), TM1-TM2 loop and TM3B. Experimental controls (I39C and G41C) are identified with red arrows. Gray box highlights the region that corresponds to background collisional level. C) Results mapped onto a molecular surface of MscS' TM segments. 


\section{III.4.3 Conformation of the N-terminal segment}

The majority of the studies performed on MscS, so far, have focused on its TM segments (Edwards et al., 2005; Miller et al., 2003a; Miller et al., 2003b; Takeshi et al., 2006a) or its large cytoplasmic domain (Koprowski and Kubalski, 2003; Schumann et al., 2004), but not on the structurally unknown $\mathrm{NH}_{2}$-terminus. The $\mathrm{MscS} \mathrm{NH}_{2}$-terminal segment, comprising 26 residues, was not resolved in the MscS crystal structure (Bass et al., 2002). However, functional experiments show that $\mathrm{NH}_{2}$-terminal truncation affects protein expression but not the ability of the channel to protect the cell during hypoosmotic challenges (Miller et al., 2003a). To establish any functional effect of the mutated cysteines on the channel function, after protein over expression, cells were subjected to a sudden osmotic downshock and cell survival assay (Vasquez et al., 2007a). The majority of the cysteine mutants (73\%) had no effect on the response of MscS to hypo-osmotic shock, 19\% had a partially impaired response (V7C, N11C, A13C, V18C, and L25C) and only 8\% experienced severe loss of function (LOF) mutants (V6C and A19C), i.e., channels that did not open upon increase in membrane tension by the osmotic downshock (Figure III.8.A). Regardless of their particular functional phenotype, all of the $\mathrm{NH}_{2}$-terminal mutants were found to be heptamers after purification. These LOF phenotypes (likely caused by channels locked in a closed state) were not disregarded in the present analysis, since this study only covers the closed conformation of MscS. 


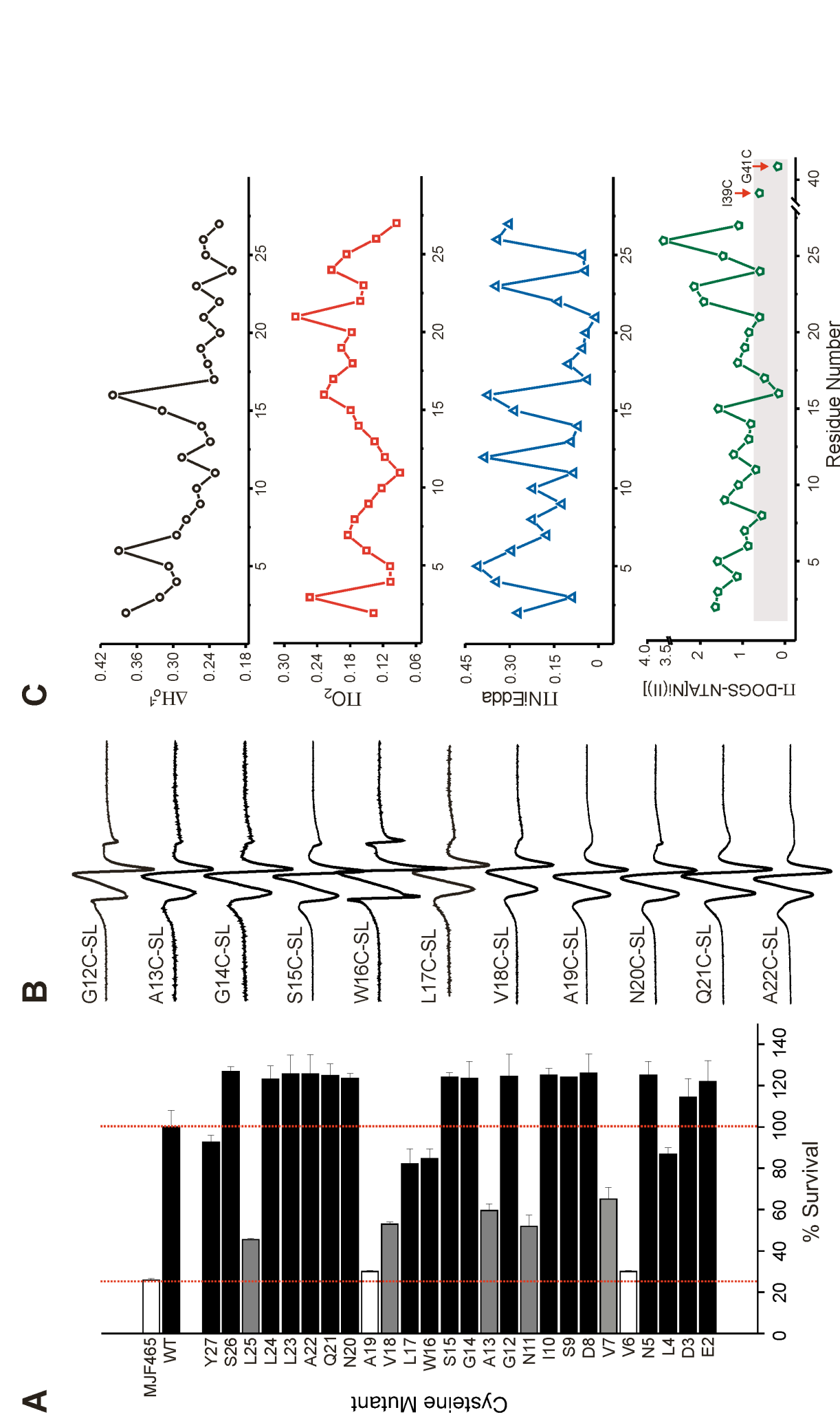

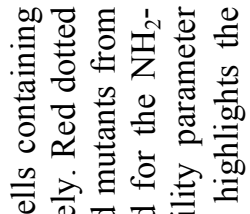

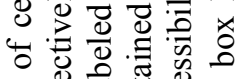

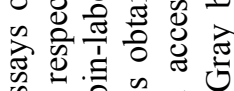

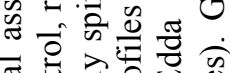
西

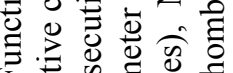

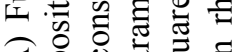
《은

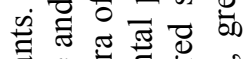
急. 胥 車要

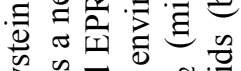
১。

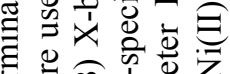
홍

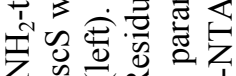
$\sum_{4} \sum_{2} \approx 0$

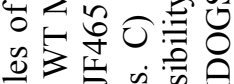

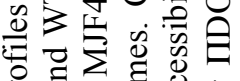

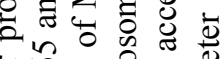

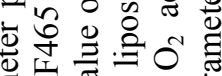

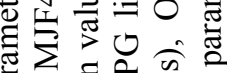
蒁 焉 은은

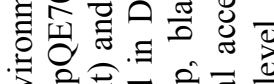

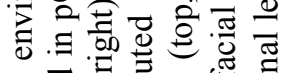
讨 ల.

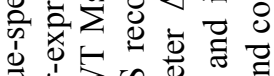

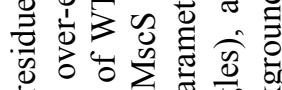
足然出

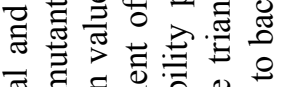
플

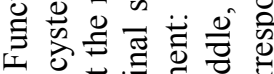

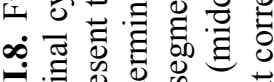

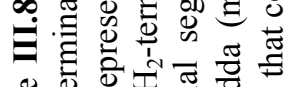
원

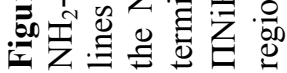


To accurately evaluate the structure of the $\mathrm{NH}_{2}$-terminal region, we used an expression vector with the His-tag on the COOH-terminus (Vasquez et al., 2007a). However, we also collected some data with the His-tag on the $\mathrm{NH}_{2}$-terminus, but the presence of the histidines along with 12 amino acid residues present in the vector linker affected the dynamical properties of this segment (Figure III.9). Under the conditions where the His-tag is on the $\mathrm{COOH}$-terminus, the $\mathrm{NH}_{2}$-terminus appears as a highly mobile fragment (Figure III.8.B, and III.11.B) with well-defined segments of secondary structure. The spectral line shape and mobility values are of the motional regime found on residues at the surface of soluble proteins (McHaourab et al., 1996). The $\mathrm{NH}_{2}$ terminus mobility profile (Figure III.8.C top panel, black trace) shows a slight decrease in probe dynamics, from residue E2C-SL to the point of initiation of TM1, with the exception of positions V6C-SL and W16C-SL, which have high mobility values, an indication of local unfolding (loop or hinge region). $\mathrm{O}_{2}$ accessibility, shown in Figure III.8.C, mainly reveals a valley (S9C-SL up to A13C-SL) and a peak (W16C-SL up to L25C-SL) of accessibilities, whereas NiEdda clearly reveals an $\alpha$-helical pattern. Calculation of the Fourier Transform power spectrum of the NiEdda accessibility profile gives a statistically significant peak of angular frequency of $100^{\circ}$ (Figure III.10), confirming the presence of $\alpha$-helical structure. Figure III.8.C (bottom panel) shows the DOGS-NTA collisional frequency suggesting that the $\mathrm{NH}_{2}$-terminus is located at the membrane interface. One segment of the $\mathrm{NH}_{2}$-terminus being exposed to the hydrated side of the membrane (positions 4-6, 12, 22-23, and 26-27) while another one is exposed to the membrane fatty acid chains (positions 3, 7-9, 17-22). S15C-SL and W16C-SL have 
high accessibilities to both contrasting agents, but W16C-SL does not have much accessibility to the Ni(II) lipid chelate, this could account for the S15C-SL facing the membrane interface and the $\mathrm{W} 16 \mathrm{C}-\mathrm{SL}$ pointing towards the pore.

We have used this set of environmental data to assign the secondary structure elements within the $\mathrm{NH}_{2}$-terminus. Figure III.11.A shows individual environmental moments for the residues in this mutant set and the vectors were superimposed on a helical wheel plot. The resultant $\mathrm{O}_{2}$ moment points to the face with the highest accessibility (positions G14C-SL, L17C-SL, and Q21C-SL) therefore to the hydrophobic part of the membrane, while the NiEdda moment is oriented about $180^{\circ}$ away, mostly defined by positions G12C-SL, L23C-SL, and S26C-SL. Overall, these results establish the $\mathrm{NH}_{2}$-terminus as an amphipathic helix lying at the membrane interface. A comparison between the $\mathrm{NH}_{2}$-terminus and the three TM segments (Figure III.11.B) leads us to suggest that this amphipathic segment, as a whole, is likely extending away from the channel pore, since its average mobility and accessibilities are higher than those of the TM domain. Application of sliding $\alpha$-periodicity index window helped us define statistically significant $\alpha$-helical regions within the $\mathrm{NH}_{2}$-terminal segment (Cornette et al., 1987; Rees et al., 1989) (Figure III.11.C). This information was used to carry out an unbiased assignment of two $\alpha$-helical segments, from I10 to G14 and N20 to Y27. These assignments served as the starting point for further refinement of our final threedimensional MscS model. 

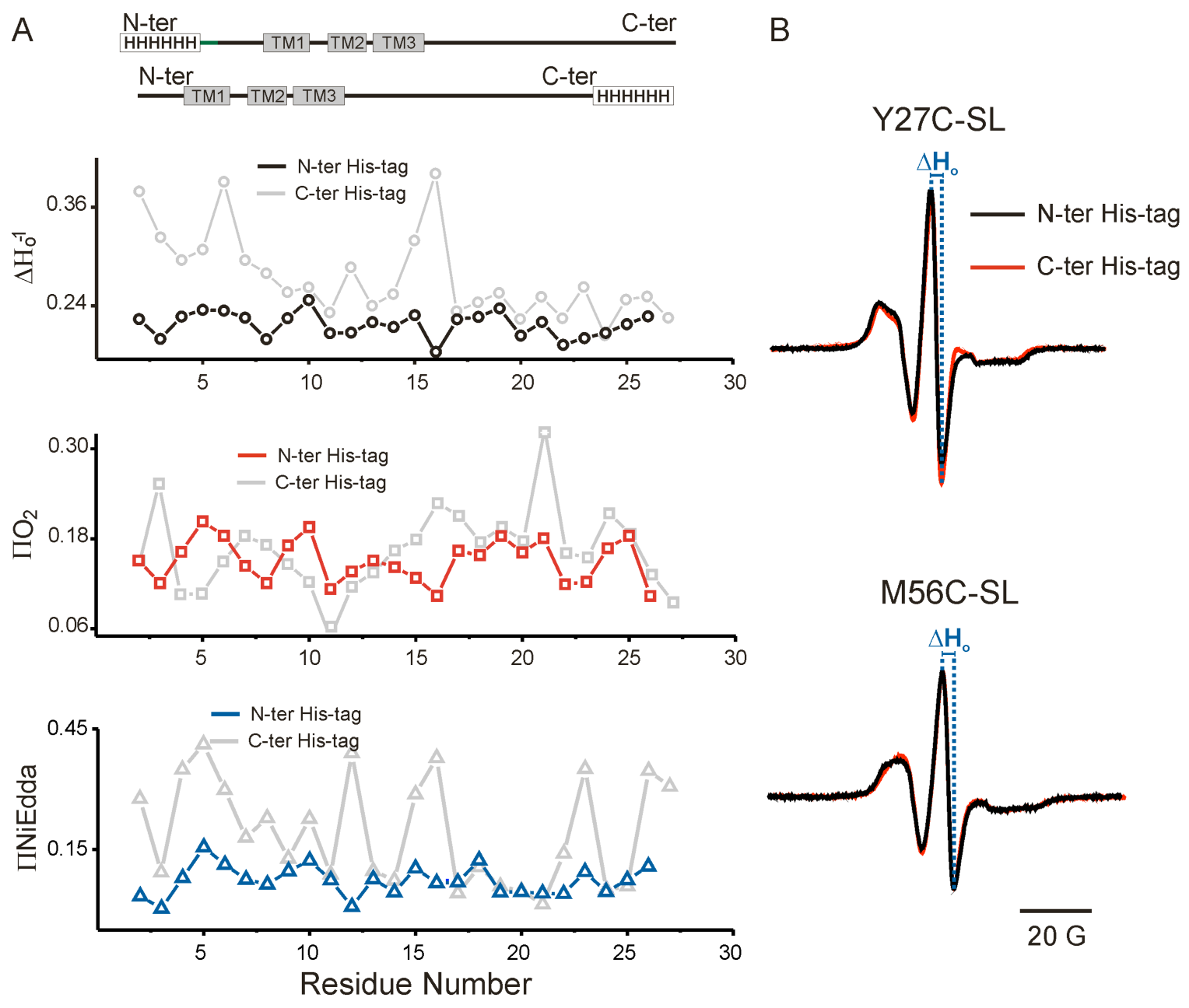

Figure III.9. EPR spectroscopy of $\mathrm{MscS}$ with an $\mathrm{NH}_{2}$-terminal His-tag. A) Residuespecific environmental parameter profiles obtained for the $\mathrm{NH}_{2}$-terminal segment expressed in pET28 (N-ter His Tag): mobility parameter $\Delta \mathrm{H}_{\mathrm{o}}{ }^{-1}$ (top, black circles), oxygen accessibility parameter $\mathrm{\Pi O}_{2}$ (middle, red squares), and NiEdda accessibility parameter INiEdda (middle, blue triangles). For comparison purposes, the environmental data obtained for MscS-pQE70, used throughout this manuscript, is plotted in light gray. B) Comparison of the X-band EPR spectra of two spin-labeled MscS TM1 mutants, purified from the N-terminal His-tag construct (black trace) and from the C-terminal His-tag construct (red trace), reconstituted in DOPC:POPG liposomes. Also indicated (in blue) is the measurement of the mobility parameter $\Delta \mathrm{H}_{\mathrm{o}}$ as the peak to peak width of the central line of the spectra. 


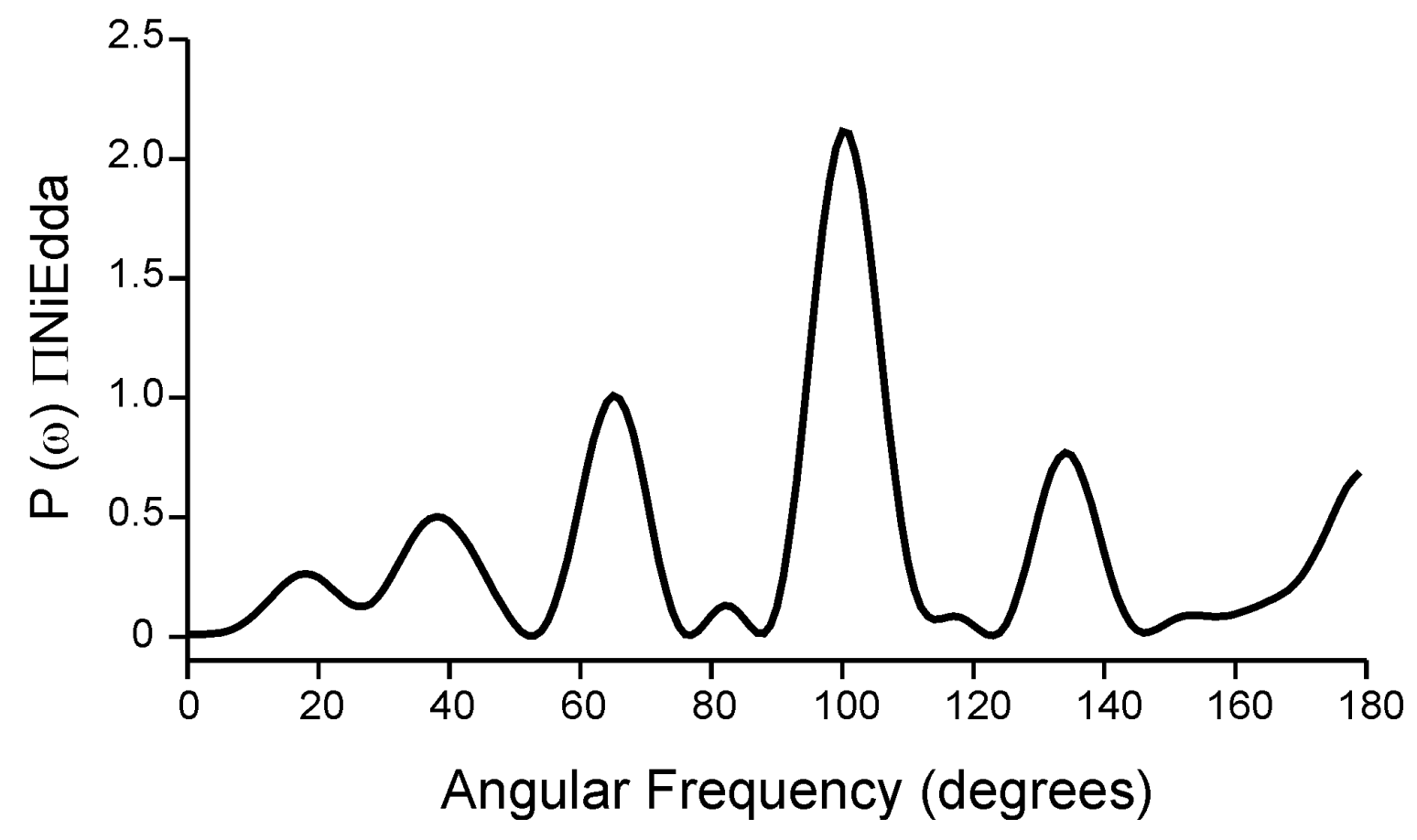

Figure III.10. Fourier transform power spectrum of the IINiEdda profile. The peak angular frequency is $100^{\circ}$, which corresponds to an $\alpha$-helical structure. 
A
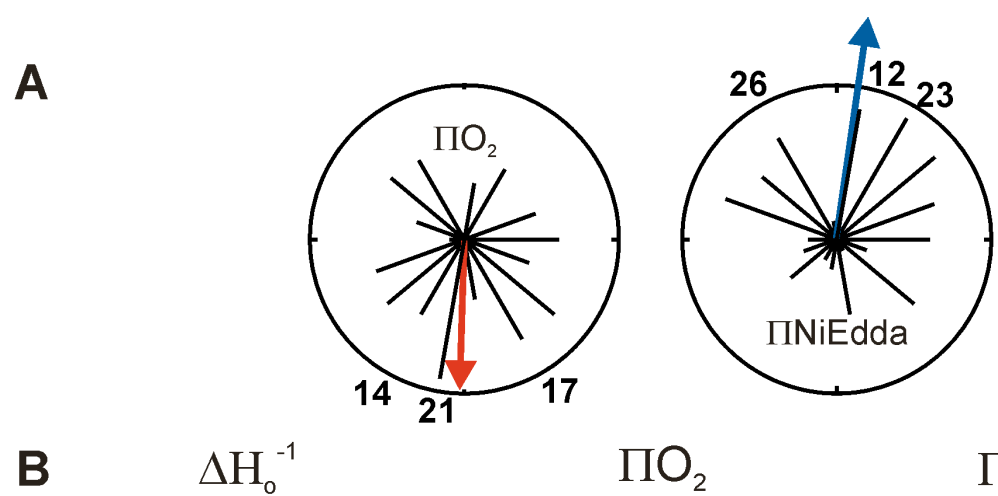

$\Pi \mathrm{O}_{2}$
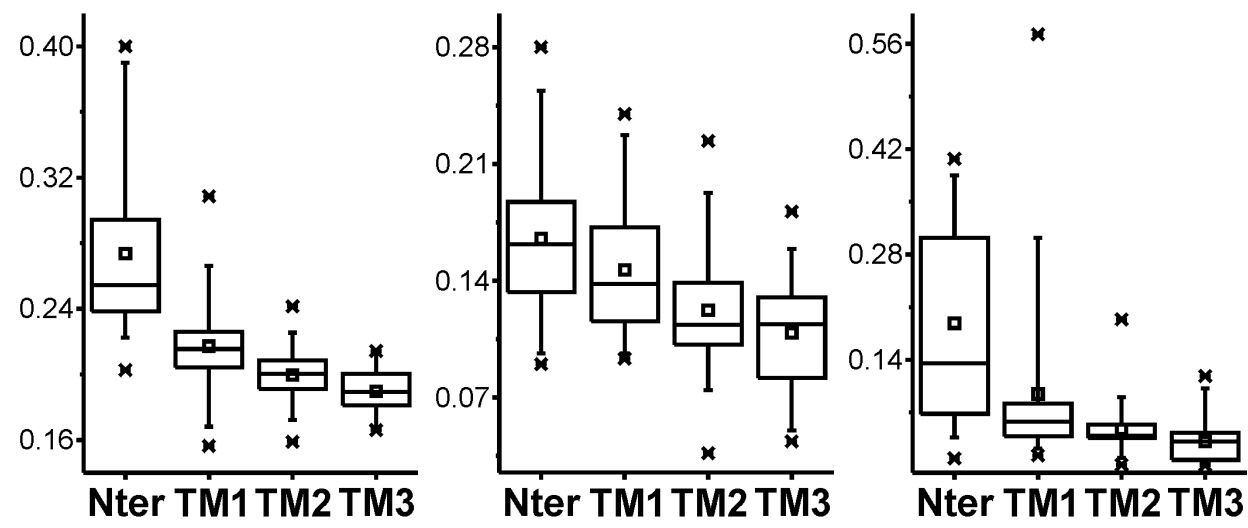

Nter TM1 TM2 TM3

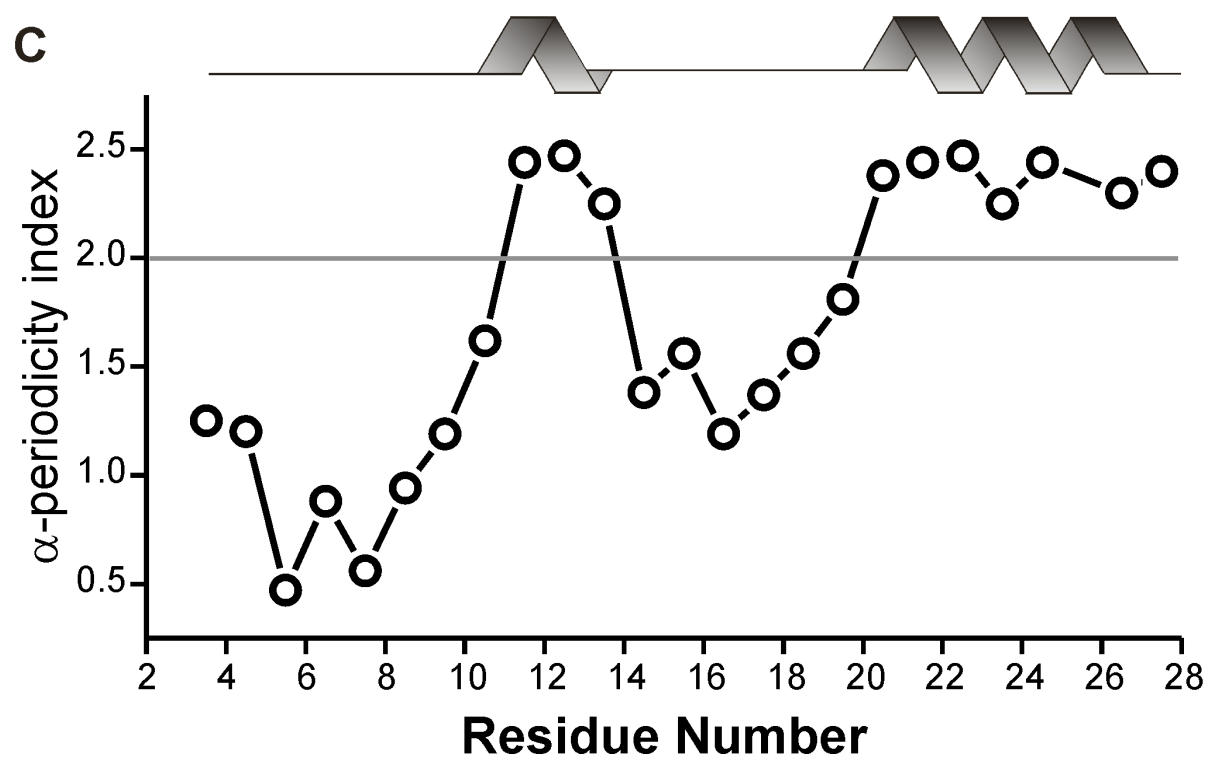

Figure III.11. Structural Analysis of the $\mathrm{NH}_{2}$-terminal segment. A) Helical wheel representation of the $\mathrm{NH}_{2}$-termini. Environmental parameters have been superimposed in a polar coordinate representation. A resultant vector was calculated for the $\mathrm{O}_{2}$ accessibility (left panel, red arrow) and NiEdda accessibility (right panel, blue arrow). B) Box plot analysis of the $\mathrm{NH}_{2}$-termini and TM segments. Squares represent the mean, boxes the data distribution, and bars the standard deviation. C) Windowed periodicity analysis for the NiEdda parameter INiEdda. The $\alpha$-helical periodicity index was calculated as described earlier (Cornette et al., 1987) with an angular range from $80^{\circ}-120^{\circ}$ and a sliding window of seven residues. The horizontal line represents the threshold at which the periodicity of the windowed segment is significantly $\alpha$-helical (10 to 14 , and 20 to 27 ). 
III.4.4 Three-Dimensional Model for the N-terminus and TM segments of MscS in the Closed State

The present data set provides information-rich constraints (128 constraints, each with three degree of freedom) to refine the molecular architecture of closed MscS in its membrane environment. Using the available crystal structure of MscS as a starting point, we generated a structural model of the $\mathrm{NH}_{2}$-terminus of $\mathrm{MscS}$ in the closed state driven by the EPR environmental data. The starting model was based on a seven-fold symmetric structure that included an $\mathrm{NH}_{2}$-terminus conformation predicted by the program Rosetta (Bonneau et al., 2002; Simons et al., 1997), as well as the MscS crystal (pdb core 2OAU (Steinbacher et al., 2007)) covering residues 27 to 178 (Figure III.12.A). Constraints on the secondary structure of the $\mathrm{NH}_{2}$-terminus ( $\alpha$-helix between residues 10 and 14, and 20 to 27) were incorporated based on frequency analysis of the angular periodicity components of the NiEdda accessibility profile (see Figure III.11.C). Structural refinement was carried out using a newly developed approach that takes advantage of EPR determined solvent accessibility restraints in MD simulations (Sompornpisut et al., 2007)(Sompornpisut et al, 2007; unpublished data). In this approach, pseudo-atoms representing EPR spin-label probes are attached to each MscS residue (from positions 1 to 178 , Figure III.12.B) and interact with pseudo-atoms that represent $\mathrm{NiEdda}$ and $\mathrm{O}_{2}$ molecules (Figure III.12.B inset). Their interactions were chosen to enforce the environments detected in the EPR experiments (aqueous, lipidic, buried in protein, membrane interface, or undetermined) as summarized in a phase diagram shown in Figure III.13. 


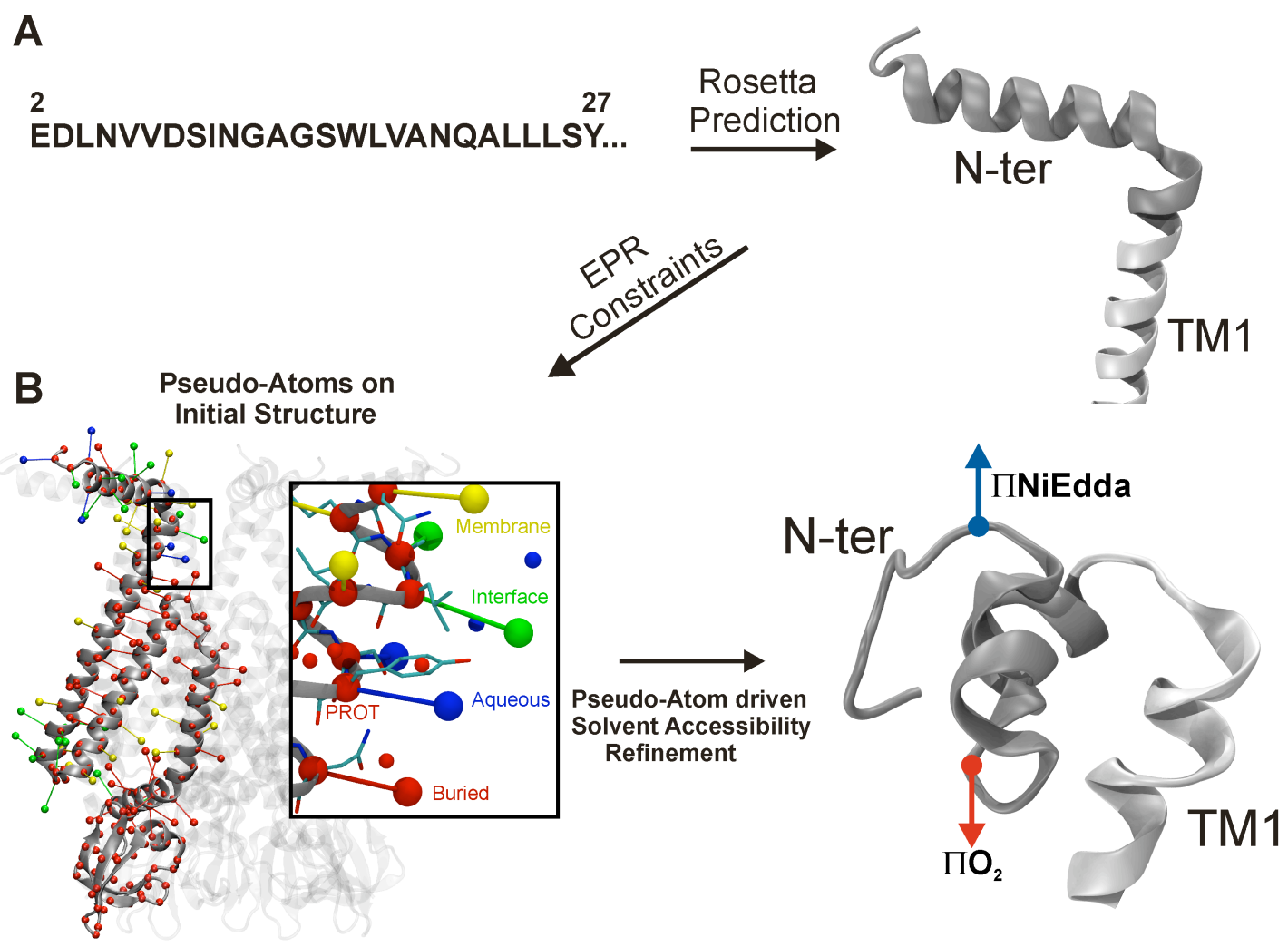

Figure III.12. Topology and structural model of $\mathrm{MscS}$ ' $\mathrm{NH}_{2}$-terminal domain, obtained by the pseudo-atom driven solvent accessibility refinement. A) Secondary structure prediction, from the linear sequence (left panel) of residues 1 to 178, using the software Rosetta (Bonneau et al., 2002; Simons et al., 1997). The right panel shows a conformation of the $\mathrm{NH}_{2}$-terminal domain obtained after energy minimization of the obtained model. The resulting structure was used in the EPR based refinement of the MscS closed conformation. B) Left panel, cartoon representation of $\mathrm{MscS}$ model used as initial structure for refinement. The model includes residues 1 to 178 . Pseudo-atoms representing the spin label attached to each residue are shown for one subunit and color coded as follows: red particles represent buried residues, blue particles represent aqueous residues, yellow particles represent residues facing the membrane, and green particles represent residues at the water-membrane interface. Red particles are shown for each $\mathrm{C}-\alpha$ atom representing PROT particles attached to each residue. Inset shows protein residues in thin licorice representation and pseudospin probes in CPK representation. Unbounded particles in red and blue represent $\mathrm{O}_{2}$ and NiEdda virtual environment particles, respectively. View of the $\mathrm{NH}_{2}$-terminal model after pseudo-atom driven solvent accessibility refinement is shown on the right panel. Residues 1 to 28 are shown in gray, resultant vectors for NiEdda and $\mathrm{O}_{2}$ accessibilities are shown in blue and red respectively. 


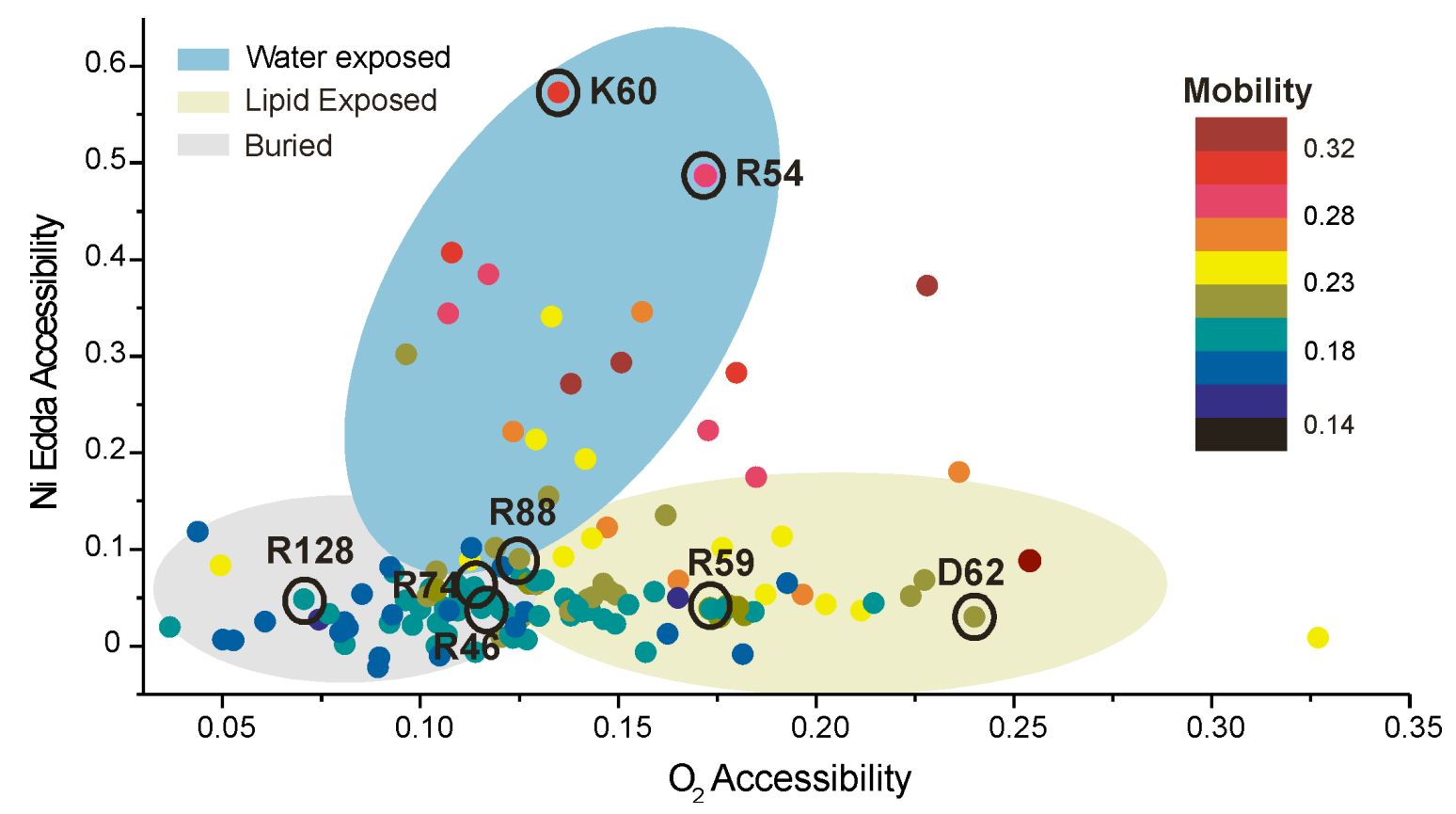

Figure III.13. Environmental phase diagram of the $\mathrm{NH}_{2}$-terminus and TM segments based on mobility parameter $\Delta \mathrm{H}_{\mathrm{o}}^{-1}$ (scale on the right), oxygen accessibility parameter $\Pi \mathrm{O}_{2}$, and NiEdda accessibility parameter $\Pi \mathrm{NiEdda}$. Relevant charged residues are indicated. Only positions with a clearly defined environment were used during refinement. 
MD simulations including the EPR constraints and probes mentioned above, together with local adjustments to ensure orientation of residues at the membrane interface, permitted a dynamic refinement of MscS. The resulting MscS model, shown in Figures III.12. B ( $\mathrm{NH}_{2}$-terminus) and III.14, satisfies the mobility and accessibility data by: compacting the TM1-TM2 helices against the pore, featuring a reorientation of these two helices (of $\sim 9^{\circ}$ ) with respect to the normal of the membrane, and narrowing the pore lined by the TM3 helices. Interestingly, within the channel context, the $\mathrm{NH}_{2}$-terminus conformation exhibits an amphipathic segment with $\alpha$-helical regions at the membrane interface, which must face away from the center of the channel in order to fit the mobility and accessibility data. As expected, the experimental data mapped onto the surfaces of the resulting model of the MscS closed state are consistent with the obtained conformation (Figures III.14.A-E).

The stability of the resulting symmetric model was then probed through all-atom MD simulations of MscS in an explicit, fully hydrated membrane environment (Figure III.15.A). The structure remained reasonably stable during six nanoseconds of free dynamics (without any symmetric constraint, Figure III.15.B). The root-mean square deviation (RMSD) of the complete model and of the TM domain alone reached values of 3.5 and $2.6 \AA$, respectively (Figure III.15.E). The larger value observed for the complete structure likely reflects the high mobility of the $\mathrm{NH}_{2}$-terminus. In fact, four out of seven subunits keep their initial architecture, but partially lose their initial secondary structure.

The RMSD values observed for the EPR-based model were found to be similar to those observed for the MscS crystal structure (pdb code 2OAU), which closes 
asymmetrically when simulated in a relaxed membrane environment (see Figure III.15.C-D). Two additional simulations of the EPR-based closed model were performed at $+/-1.2$ Volts (3 ns each). The simulations demonstrated that the structure indeed represents a conformation of very low conductance (data not shown). Water penetration into the periplasmic mouth of the channel observed throughout simulations, along with the NiEdda accessibility data suggests that the periplasmic channel region might be even more occluded than what the EPR-based model predicts. Finally, it is interesting to note that the model predicts formation of a salt bridge between residues 62 and 128 of adjacent subunits, likely relevant for MscS function (Sotomayor and Schulten, 2004). 
A

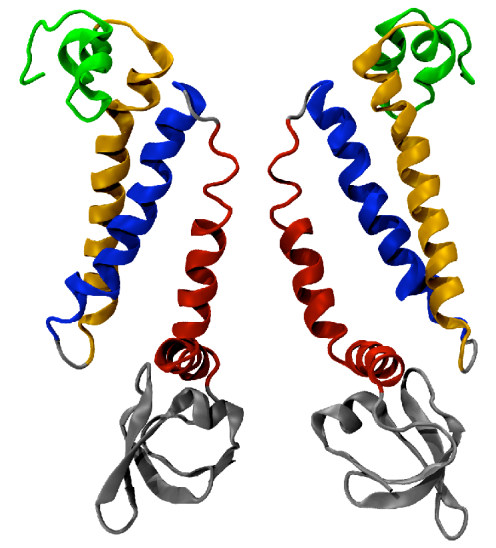

C

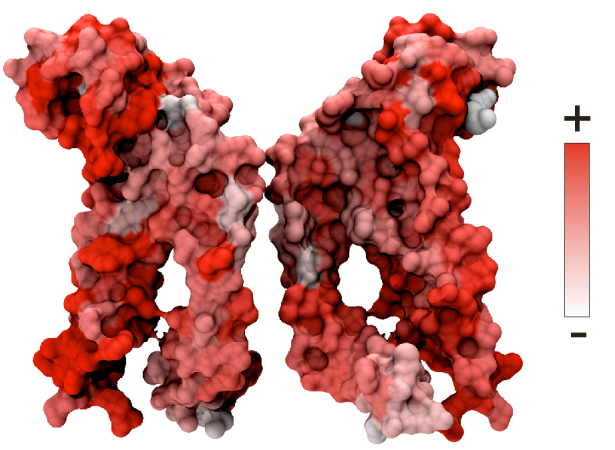

B

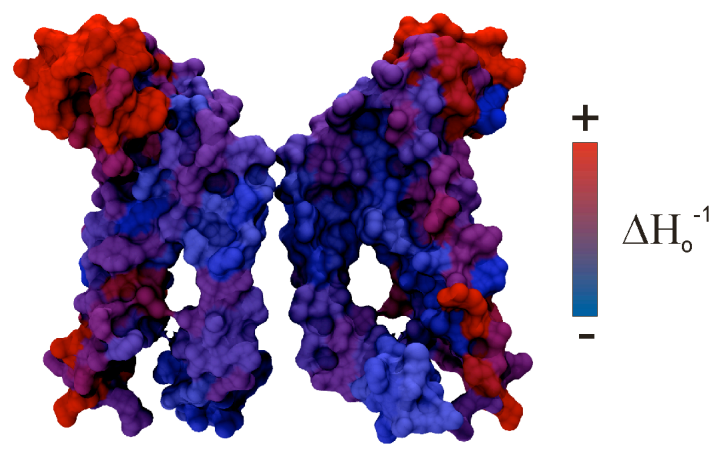

$\mathrm{D}$

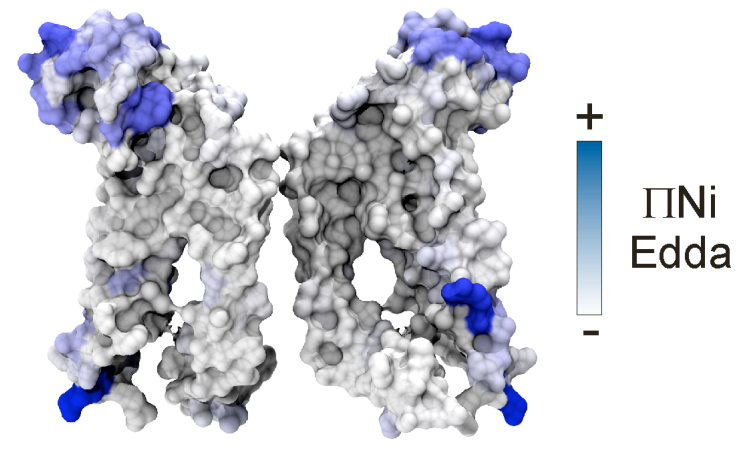

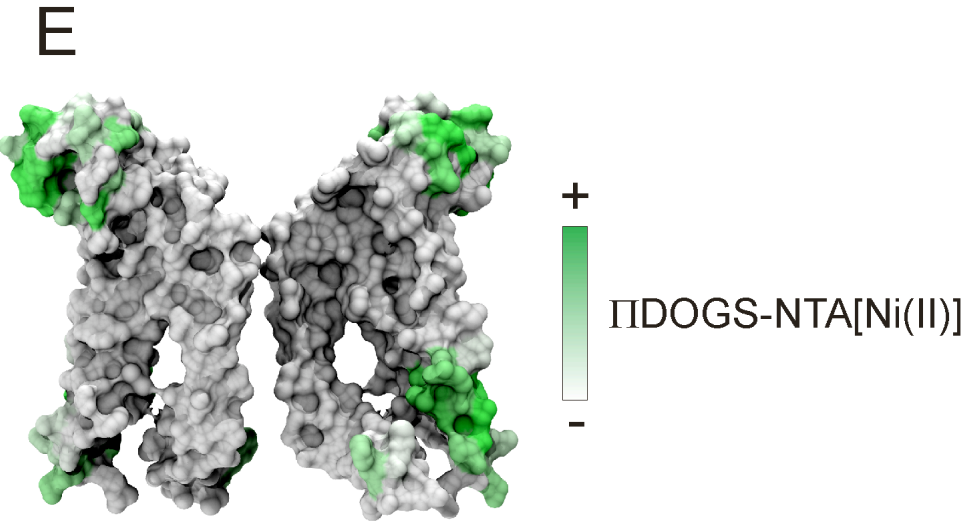

Figure III.14. MscS closed conformation obtained from EPR based refinement. A) Ribbon representation of the MscS closed state model (two subunits are shown for clarity). Individual TM segments are color-coded as follows: $\mathrm{NH}_{2}$-terminus, green, TM1, yellow; TM2, blue; and TM3, red. BE) Mobility parameter $\Delta \mathrm{H}_{0}^{-1}, \mathrm{O}_{2}$ accessibility parameter $\Pi_{2}$, NiEdda accessibility parameter חNiEdda, and interfacial accessibility parameter חDOGS-NTA[Ni(II)]lipids, respectively. 


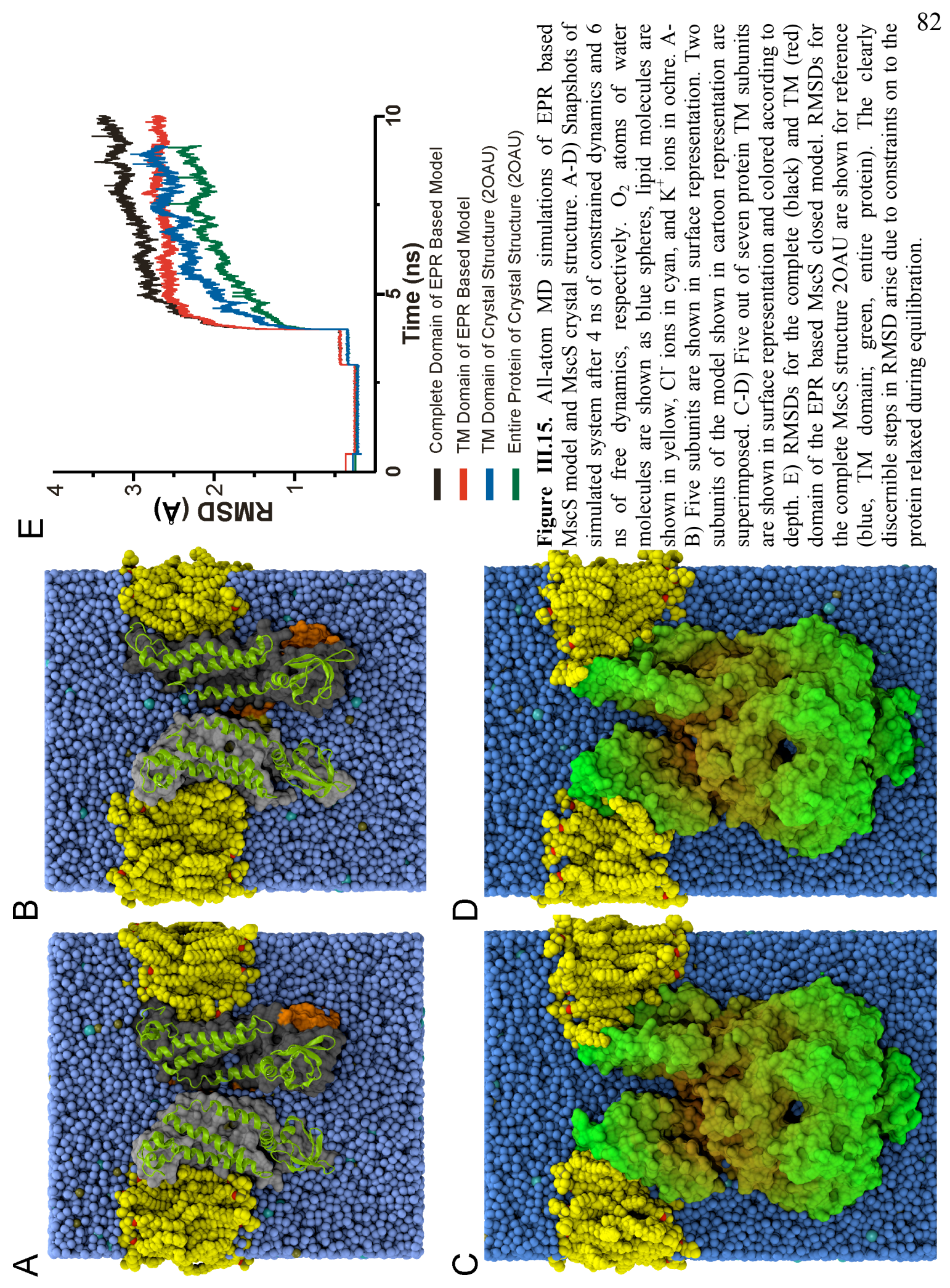




\section{III.5 DISCUSSION}

Membrane proteins are a fundamental part of the cell machinery, serving as switches, sensors, and bridges between the extracellular and intracellular space. However, establishing the correlation between structure and mechanism is fraught with difficulty. X-ray crystallography can provide a detailed atomistic view of a membrane protein, but crystallization in absence of a native-like lipid environment has the potential to induce a non-physiological conformation (Jiang et al., 2003). Particularly, for the MscS channel it is very important to know its conformation in the membrane, since its gating relies on the direct interaction with the lipid bilayer (Takeshi et al., 2006a). Determining what functional state is depicted by the crystal conformation is extremely challenging, particularly when large conformational changes arise from external stimuli. MS channels undergo such large rearrangements in their structure upon application of mechanical stimuli, as has been shown in MscL (Perozo et al., 2002a). Unambiguous identification of MscS's closed, open, and inactive conformations is an essential step in the quest to understand its gating mechanism and function in bacterial cells and mechanosensation, in general.

The present chapter focuses on determining the three-dimensional fold of $\mathrm{MscS}$ $\mathrm{NH}_{2}$-terminus and TM domain, when MscS is in its closed conformation, embedded in a lipid bilayer. EPR spectroscopy analysis of spin labeled mutants provided information on the topology, secondary, and tertiary structure of MscS under native conditions. This information was used, along with MD simulations, to evaluate a recently further refined crystal structure (Steinbacher et al., 2007) and propose a model of MscS in a closed conformation. 
The EPR data shows that there are general similarities between the overall structure and topology of $\mathrm{MscS}$ in a biological membrane and in the crystal conformation. However, some differences point to the fact that the MscS crystal structure does not represent the true closed state, unlike MscL where differences between the crystal structure and the one suggested by the EPR data are more subtle (Perozo et al., 2001). The accessibilities to the water-soluble compound NiEdda indicate that positions at the cytoplasmic end of TM1, that were predicted to be in contact with fatty acid chains, were able to collide with this aqueous reagent. Additional evidence for the lipid exposure of $\mathrm{MscS}$ came from the results obtained with the DOGS-NTA[Ni(II)]lipids. These results, when combined with information obtained from mobility, $\mathrm{O}_{2}$, and $\mathrm{NiEdda}$ accessibilities, clearly localizes the protein within the lipid bilayer. The thickness of a DOPC membrane, $50 \AA$ (Wiener and White, 1992), was used along with the accessibilities obtained with DOGS-NTA[Ni(II)]lipids to determine how MscS sits in the lipid bilayer.

According to the crystal structure, the TM2 helix should be asymmetrically solvated, i.e., one surface of this helix should be facing TM1 (buried), while the opposing surface should be solvated by lipids found in crevices between TM1-TM2 hairpins. However, our model suggests that, in the closed state, the TM2 $\mathrm{NH}_{2}$-terminal faces the lipid bilayer while its $\mathrm{COOH}$-terminal region is less exposed to the membrane and likely faces TM3, placing TM2 spatially closer to TM3 than what is predicted by the crystal. The TM2 helix is also predicted to be longer, beginning at position T64 instead of F68 (crystal). It remains to be elucidated if the rotation in the membrane plane of the TM3B 
helix (Figure III.16.B) affects the architecture of the cytoplasmic domain and its openings.

While overall exposure to $\mathrm{O}_{2}$ and NiEdda in TM3 is low when compared to TM1 and TM2, a patch of higher $\mathrm{O}_{2}$ accessibilities than the TM3 average (positions 104 to 112), was observed (Figure III.5.B-C). This hydrophobic area could account for a partially open pore but functionally closed with a region of the permeation path that contains no water and, thus, blocks ion conduction. Multiple computational studies of water dynamics inside $\mathrm{MscS}$ have shown that such scenario is possible (Anishkin and Sukharev, 2004; Sotomayor and Schulten, 2004; Sotomayor et al., 2007; Spronk et al., 2006) (Figure III.15.B). Other ion channels trapped in the closed conformation such as MscL (Chang et al., 1998), the nicotinic acetylcholine receptor (Miyazawa et al., 2003), KcsA (Doyle et al., 1998) and KirBac1.1 (Kuo et al., 2003), have been shown to maintain a non-conducting pore even if the dimensions of the permeation path are large enough to allow passage of dehydrated ions.

The MscS $\mathrm{NH}_{2}$-terminal segment model presented here forms a cap at the periplasmic face of the channel. In order to satisfy our mobility and accessibility data the final model displays an $\mathrm{NH}_{2}$-terminal domain that points away from the permeation pathway and includes $42 \%$ of $\alpha$-helical structure. The function of this segment is unknown, but substitutions at W16 affects the response to negative pressure under patch clamp conditions (Rasmussen et al., 2007), and deletions on the $\mathrm{NH}_{2}$-terminal segment affect the organization, and perhaps incorporation, of $\mathrm{MscS}$ in the lipid bilayer (Miller et al., 2003a). We can then suggest that, the $\mathrm{NH}_{2}$-terminal could also play a potential role in membrane-modulated gating due to its amphipathic nature. 

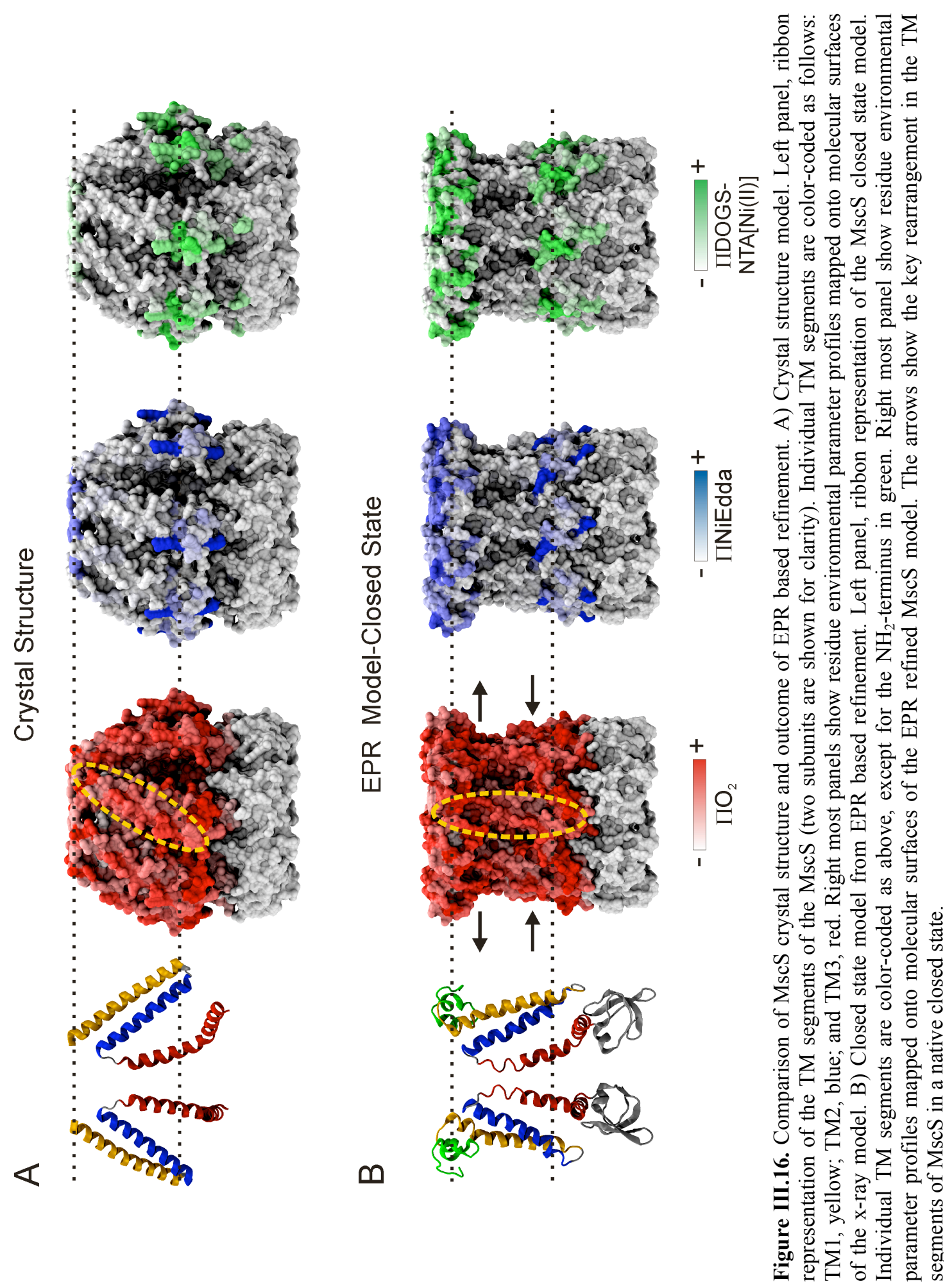
Using a novel refinement method with energy restraints exploiting the information from EPR data, we have rearranged the x-ray structure into a model that represents the closed state. While the final model incorporates as much information as possible from the experiments, there are two limitations of the modeling process that should be noted. First, prediction of a three-dimensional model based only on sequence is difficult, particularly for a multimeric membrane protein. Second, the EPR derived data presented here represents an average over a large population of channels and, therefore, asymmetry as observed in the crystal structure and MD simulations (Sotomayor and Schulten, 2004; Sotomayor et al., 2006; Sotomayor et al., 2007; Spronk et al., 2006) cannot be detected. Thus, the presented model is a symmetrized version of $\mathrm{MscS}$. Nevertheless, the power of our approach comes from the pattern of global accessibilities more than the absolute values of a given position, along with the fact that the membrane has well defined environments that reduce the number of models that can properly fit the experimental constraints. Further structural, spectroscopical, and computational studies will be required to overcome these limitations and to also establish the open state of MscS, and whether the conformation depicted by the crystal structure is physiologically relevant.

A summary of the proposed closed conformation of the MscS TM domain in a lipid bilayer and a comparison to the crystal conformation is presented in Figure III.16. TM helices are more perpendicular to the plane of the membrane than the helices in the crystal structure. TM1 has two faces, one exposed to the membrane and the other one to TM2. TM2 is surrounded by TM1 and TM3, except for its cytoplasmic region that has some exposure to the membrane. In our refined model, the pore is apparently narrower, 
but how much closer the TM3 helices are to each other cannot be determined in a quantitative manner. The nine-degree inward motion of TM1-TM2 favoring a more compact structure, and the almost horizontal TM3B helix observed during refinement are both in agreement with the motions observed in previous MD studies (Sotomayor and Schulten, 2004).

Finally, the present study also provides some hints to an additional, physiologically relevant function of $\mathrm{MscS}$. Some cysteine mutants produced twice the amount of cells than the WT and other cysteine mutants (after IPTG induction). The respective residues, most of them hydrophobic (W16, V32, V40 and A79), are conserved throughout the MscS family (Bass et al., 2002). Residue W16 has already been shown to participate in the positioning of MscS on the membrane (Miller et al., 2003a), and it does not seem to dramatically affect MscS function (see Figure III.8.A, refs. Miller et al., 2003a, and Rasmussen et al., 2007). In contrast, when residue V40 was substituted by aspartic acid or lysine, a significant GOF phenotype (in which cells were not able to grow) was observed, whereas cysteine substitution was tolerated by the cell (Okada et al., 2002). Additionally, cells lacking MS channels exhibit a faster growth rate than other $E$. coli strains (data not shown). Eukaryotic MS channels have been shown to play an important role during mitosis, as the mechanical properties of their membrane changes during the cell cycle (Haswell and Meyerowitz, 2006; Rundle et al., 2004; Zhou and Kung, 1992). Perhaps MscS also plays a role in bacterial cell division. 
CHAPTER IV: Three Dimensional Architecture of Membrane-Embedded MscS in the Open Conformation. Conformational Rearrangements in MscS

\section{IV.1 ABSTRACT}

The mechanosensitive channel of small conductance (MscS) belongs to a large and widespread family that is distributed among prokaryotes, archaea, and plants. Earlier EPR analysis of MscS in the closed conformation showed that in a membrane environment, MscS displays a significant reorientation of the transmembrane helices towards the bilayer normal, forming a more compact structure when compared to $\mathrm{MscS}$ crystal structure. Here, we have trapped the open conformation by modulating the membrane pressure profile. By EPR spectroscopy we have determined that the transition from the closed to the open state is accompanied by an increase in overall dynamics that involves significant rearrangements such as burying of the $\mathrm{NH}_{2}$-terminus in the membrane, tilting of TM1-TM2 segments, expansion, rotation, and wetting of the TM3 helices. Our data from the closed and open states allows us to establish the overall conformational changes of the transmembrane domain during MscS gating in a lipid environment. 


\section{IV.2 INTRODUCTION}

Mechanosensation is involved in many physiological roles, and frequently relies on the presence of mechanosensitive ion channels. The mechanosensitive ion channel family members are grouped by function rather than topology, since there is no consensus sequence among them (Kloda and Martinac, 2002; Martinac, 2001; Pivetti et al., 2003). There are currently available three mechanosensitive channel crystal structures; two of them belong to the prokaryotic family, MscL (Chang et al., 1998; Steinbacher et al., 2007) and MscS (Bass et al., 2002; Steinbacher et al., 2007), and ASCI1 that belongs to the eukaryotic epithelial sodium channel/degenerin family (Jasti et al., 2007). It still remains to be determined if the tension-sensing process is conserved throughout this heterogeneous super-family.

A considerable amount of information has been gathered on MscL and MscS over the past few years. Both channels have been electro-physiologically characterized (Akitake et al., 2005; Berrier et al., 1996; Koprowski and Kubalski, 1998; Sukharev, 2002; Sukharev et al., 1993), and their crystal structures has been determined, yet the gating mechanism is not fully understood, which is required to define common elements in mechanosensation. MscL has been used as a model to understand lipid-protein interactions since its molecular mechanism of gating has been elucidated (Iscla et al., 2007; Perozo et al., 2002a; Sukharev et al., 2001a; Sukharev et al., 2001b). Even though MscL is a fantastic tool to study the mechanical properties of the lipid bilayer in determining the function of the proteins, the sequenced members of the MscL family are currently restricted to one archaeon, a single fungus, and bacteria, while the MscS family is much more widely distributed in the three domains of life (Pivetti et al., 2003). 
A lot of effort has been made to address the physiological states represented by MscL and MscS x-ray structures, since they were determined in detergent micelles and their activation solely depends on the membrane lateral tension. Unlike MscS, MscL crystal structure is a good representation of the closed conformation in its native environment (Gullingsrud et al., 2001; Perozo et al., 2001). Until recently, there where many doubts about the conformation depicted by $\mathrm{MscS}$ structure, but several experimental and computational analysis have confirmed that MscS crystal structure does not represent the open nor the closed state (Anishkin et al., 2008; Anishkin and Sukharev, 2004; Sotomayor and Schulten, 2004; Sotomayor et al., 2006; Sotomayor et al., 2007; Spronk et al., 2006; Vasquez et al., 2007b). We have recently determined the threedimensional architecture of $\mathrm{MscS}$ in the closed conformation in a lipid bilayer (Vasquez et al., 2007b). The EPR spectroscopic analysis of lipid-reconstituted MscS in the closed state revealed a more compact TM domain than in the crystal structure characterized by a realignment of the TM segments towards the normal of the membrane, and also locates the previously unresolved $\mathrm{NH}_{2}$ terminus within the bilayer interface. Yet, the closed conformation only represents the ground state in the determination of the molecular mechanism of MscS gating.

In the present study, we have stabilized the open conformation of MscS by modifying the transmembrane tension profile through the incorporation of a cone-shaped amphiphile. Thus, we have concentrated our efforts on monitoring the structural rearrangements upon gating of MscS TM domain. All patch-clamp experiments were performed on WT MscS, while EPR experiments were done on MscS spin-labeled cysteine mutants (positions 2 to 128). The data was obtained under conditions where the 
internal bilayer profile and the membrane torque were modified without the application of tension. This approach has been already established to be successful in the study of MscL gating mechanism (Perozo et al., 2002a; Perozo et al., 2002b), where the transbilayer lateral tension was modified by the addition of a cone-shaped phospholipid (lysophosphatidylcholine, lyso PC). We are currently using the pattern of global accessibilities and mobilities obtained by CW-EPR spectroscopy to build a threedimensional model of $\mathrm{MscS}$ in an open conformation in order to elucidate the conformational changes during gating. Analysis of individual mutants allow us to propose that upon opening the $\mathrm{NH}_{2}$ terminus of $\mathrm{MscS}$ penetrates into the membrane, the TM1-TM2 hairpin tilts and moves away from the TM3 segment, the pore increases its diameter, the TM3 helices rotate, and wetting of the permeation pathway occurs. This collection of data from the closed and open states provides a physical framework to understand the activation gating of $\mathrm{MscS}$. 


\section{IV.3 EXPERIMENTAL PROCEDURES}

\section{IV.3.1 Spheroplast Patch-Clamp}

Channel activities were recorded by patch clamping giant spheroplasts, following the method of refs. Kuo et al., 2007 and Martinac et al., 1987. Patch Clamp measurements were done, in the inside-out configuration, under symmetrical conditions (200 $\mathrm{mM} \mathrm{KCl}, 90 \mathrm{mM} \mathrm{MgCl} 2,10 \mathrm{mM} \mathrm{CaCl}_{2}$, and 10mM HEPES), at $\mathrm{pH} 7.5$ at room temperature. The pipette and the bath solutions contained $500 \mathrm{mM}$ sucrose, to keep spheroplasts integrity.

The pipettes were obtained from glass capillaries (SIGMA, catalog number P1174), and were fire polished before used, until it was reached a resistance between 2 and 2.5 M $\Omega$. Negative pressure on the patch was obtained by applying suction through a pressure-clamp system (HSPC-1, ALA Scientific Instruments). To activate the channels without negative pressure, $3 \mu \mathrm{M}$ of 18:1 lyso PC (1-Oleoyl-2-Hydroxy-sn-Glycero-3Phosphocholine, from Avanti Polar Lipids) was added to the bath solution. Macroscopic currents were recorded with a DAGAN 3900 patch-clamp amplifier, and currents were sampled at $40 \mathrm{KHz}$ with an analog filter set to $5 \mathrm{KHz}$. Analyses were done using pCLAMP9 (Axon Instruments).

\section{IV.3.2 Mutagenesis, expression, spin labeling, and reconstitution of MscS}

Cysteine mutants were generated for residues 2-128 in $\mathrm{MscS}$, covering the $\mathrm{NH}_{2}$ terminus, TM1, TM2, and TM3. Mutagenesis was performed using the QuickChange ${ }^{\mathrm{TM}}$ Site-Directed Mutagenesis Kit (Stratagene). Mutant channels were expressed, purified, labeled and reconstituted as previously described (Vasquez et al., 2007a) using a pET28 vector containing MscS with His6 epitope at the $\mathrm{NH}_{2}$-termini and a $\mathrm{pQE70}$ vector with 
His6 epitope at the COOH-termini, for the study of the TM segments and the $\mathrm{NH}_{2}-$ terminus, respectively. MscS-pET28 was used to express the channel in E. coli Roseta $^{\mathrm{TM}}$ and MscS-pQE70 was used for expression in E. coli M15. The samples were reconstituted at a 750:1 lipid/channel (DOPC:POPG, 6:1) molar ratio by dilution in PBS in the presence of Bio-beads.

\section{IV.3.3 EPR spectroscopy and data analysis}

All EPR data were obtained at room temperature. X-band CW EPR spectra were performed as previously described (Perozo et al., 1998). Spectra were obtained in a Bruker EMX spectrometer fitted with a dielectric resonator under the following conditions: $2 \mathrm{~mW}$ incident power, $100 \mathrm{kHz}$ modulation frequency and $1 \mathrm{G}$ modulation. The accessibility parameters were quantified as previously reported (Farahbakhsh et al., 1992; Gross and Hubbell, 2002; Perozo et al., 1998; Vasquez et al., 2007b). 


\section{IV.4 RESULTS}

For this study, we used the set of individual spin-labeled cysteines mutants of MscS TM domain, previously utilized. This group of mutants was expressed, purified, labeled, reconstituted and characterized as previously reported (Vasquez et al., 2007a; Vasquez et al., 2007b). Figure IV.1.A shows the mutated residues (2-128) as black spheres on a ribbon diagram of the $\mathrm{MscS}$ monomer that includes the previously unresolved $\mathrm{NH}_{2}$-terminus, according to the EPR-based closed state model.

\section{IV.4.1 Stabilization of MscS open state by asymmetric incorporation of} lysophosphatidylcholine

To evaluate the functional consequences on MscS upon lyso PC incorporation into the membrane, we have first carried out patch-clamp experiments on WT MscS, expressed in giant spheroplasts (Figures IV.1.B and IV.1.C). We chose to use this system (more stable and reproducible under patch-clamp experiments) to determine the effect of this amphipath on MscS, since it has been already established that MscS conductance and tension dependence is essentially the same in E. coli spheroplasts than in reconstituted systems (Machiyama et al., 2008; Sukharev, 2002; Vasquez et al., 2007a). Prior addition of lyso PC, we determined the presence of MscS currents by applying negative pressure at $+10 \mathrm{mV}$ TM potential. Figure IV.1.C shows a representative trace where it becomes evident that MscS upon sustained negative pressure inactivates with a time constant in the order of seconds. These pressure pulses were repeated every 3 minutes (at least six times), so we could recover channels from inactivation (Akitake et al., 2005; Sotomayor et al., 2007), and determine the amount of channels present in the patch (approximately 55). After addition of lyso $\mathrm{PC}$ in the absence of applied pressure, at $+10 \mathrm{mV}$, we 
observed spontaneous opening of MscS (right panel, Figure IV.1C). In these conditions, the single channel events showed the same conductance than when negative tension is applied (inset, Figure IV.1C). After approximately two minutes, more channels were activated until steady state was reached. Since, we knew that not all of the channels present in the patch were opening we added more lyso PC to recruit them all. Roughly two minutes later we recorded an abrupt change in current, which corresponded to activation of more channels, until the membrane seal broke down. Our results demonstrate that the incorporation of lyso PC in vitro activates MscS and does not allow it to visit the inactivated state. The lack of inactivation on $\mathrm{MscS}$ under this experimental condition makes lyso PC a great tool that would allow us to determine the open conformation rather than the inactivated one, by EPR spectroscopy. 
A

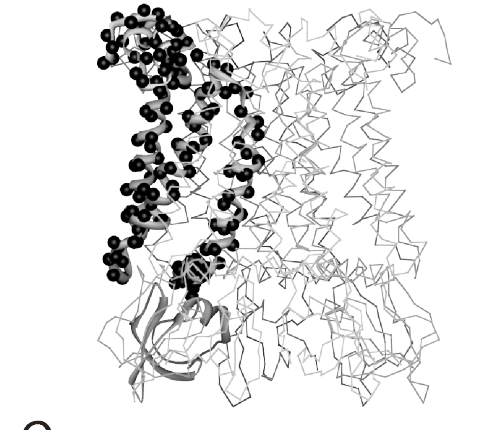

C
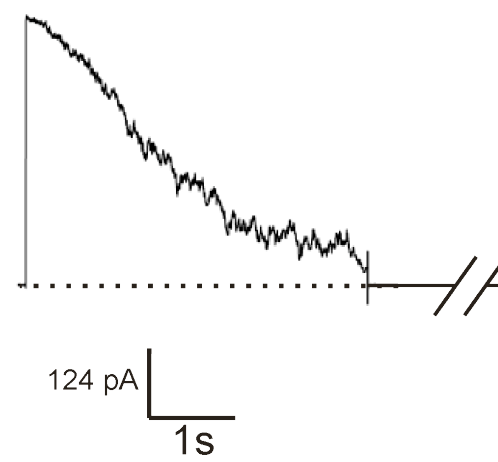

B

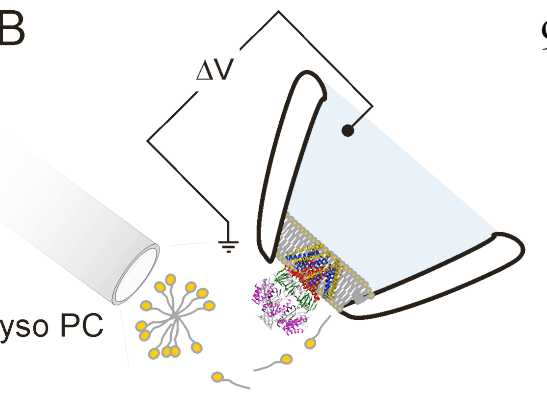

97

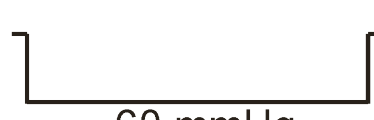

$-60 \mathrm{mmHg}$

Figure IV.1. MscS does not inactivate upon lyso PC activation. A) Single MscS transmembrane domain subunit showing amino acid residues (black spheres) probed through site directed spin labeling of cysteines. A single MscS monomer is represented as part of the heptamer according to the $\mathrm{MscS}$ closed state model, from the EPR based refinement. B) Diagram represents $\mathrm{MscS}$ orientation in the inside-out patch-clamp configuration (in the absence of applied tension), on the presence of lyso PC micelles. C) Left panel, representative MscS macroscopic currents activated by negative pressure $(-60 \mathrm{mmHg})$, at $+10 \mathrm{mV}$, reveal the presence of a time-dependent inactivation process (with a time constant of $1.5 \mathrm{~s}$ ). Middle panel, asymmetric incorporation of lyso PC, in the absence of applied tension at $+10 \mathrm{mV}$, elicits spontaneous opening after $\sim 2$ minutes of its addition $(3 \mu \mathrm{M})$. The single-channel conductance, calculated from the traces shown in a slow time scale and as a higherresolution detail, corresponds with previous electrophysiological measurements. Left panel, macroscopic activation of $\mathrm{MscS}$ elicited by the incorporation of lyso PC. Once channel activity reached steady state more lyso PC was added $(3 \mu \mathrm{M})$; after $\sim 2$ minutes of its addition more channel activity was recruited. The number of channels activated by negative pressure (right panel) and by lyso PC incorporation (left panel) was approximately the same. 


\section{IV.4.2 Structural Rearrangement of MscS Transmembrane Domain upon}

\section{Opening}

In order to determine the maximal response of $\mathrm{MscS}$ upon lyso PC addition, we used three positions covering the full length of MscS TM1 (N30C, L42C, and N50C). In contrast to the large structural changes observed in MscL (Perozo et al., 2002b), addition of lyso PC to the liposome suspension produced smaller changes in overall line shape of TM1 spin-labeled residues (Figure IV.2.A). This outcome is somewhat anticipated, given the smaller single channel conductance of MscS (respect to MscL) and the fact that being a homoheptamer, small inter-subunit movements could generate the relatively large radial pore changes necessary for ion conduction. Nevertheless, dose-response curves were obtained for positions N30C-SL and N50C-SL from changes in the mobility parameter (Figure IV.2.B). In both cases, increasing addition of lyso PC produced a sigmoid enhance in mobility with a mid-point around 8-10\%. This effect suggests a global conformation rearrangement from individual spin labels, except for position L42C-SL (in the middle of TM1) that as expected has subtler changes.

We systematically added $25 \mathrm{~mol} \%$ of lyso PC (saturating concentration, Figure IV.2.A) to each reconstituted spin-labeled cysteine mutant, in order to obtain the pattern of overall mobilities and accessibilities of $\mathrm{MscS}$ in an open conformation. This global pattern was experimentally determined by defining the local environment surrounding a given spin-label, using: probe mobility $\left(\Delta \mathrm{H}_{\mathrm{o}}^{-1}\right)$, and accessibilities ( $\left.\Pi\right)$ through paramagnetic species that quench the spin-label signal, such as $\mathrm{O}_{2}$ (highly soluble in the non-polar region of the bilayer), and NiEdda (a polar compound mainly present in the aqueous media). 
A

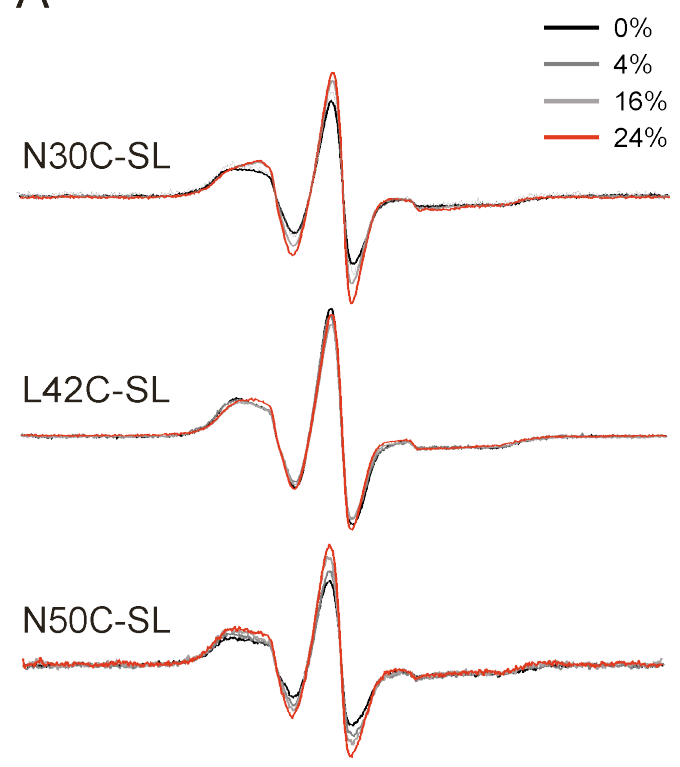

B

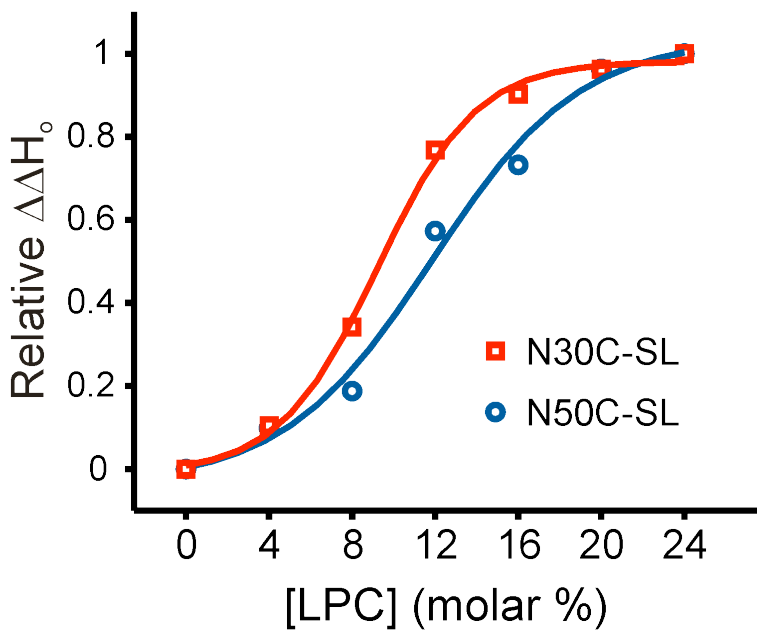

Figure IV.2. Asymmetric incorporation of Lyso PC induces conformational changes in MscS. A) CW-EPR spectra for TM1 spin-labeled residues N30C, L42C, and N50C obtained at increasing concentrations (molar \%) of Lyso PC. Spectral width is $150 \mathrm{G}$. B) Relative changes in spin-label mobility $\left(\Delta \Delta \mathrm{H}_{0}\right)$ as a function of Lyso PC molar percent. Dose-response fits to positions 30 and 50 revealed a Lyso PC midpoint effect of approximately 8 and $10 \%$, respectively 
Figure IV.3.A shows a representative set of residues spectra that line the permeation pathway (G101C-SL to G113C-SL), with an almost uniform sharpening of the spectral line shape, representing a significant increase in probe dynamics. The entire EPR data set, for the TM domain including the $\mathrm{NH}_{2}$-terminus, is shown in Figure IV.3.B. In MscS, the transition to the open state results in an overall increase in mobilities and $\mathrm{O}_{2}$ accessibilities for most of the TM domain. However, not all of the TM segments equally respond to the incorporation of lyso PC. Upon opening, the first six residues of the $\mathrm{NH}_{2}$ terminus decreased their dynamics, the central portion of TM1 (from position I38C-SL to I44C-SL, broken red curve on top panel of Figure IV.4) did not show significant changes, whereas the TM2 and TM3 segments (previously constricted in a proteinaceos environment in the closed state) gained mobility, and the TM2, in particular, seems to be more exposed to the lipids. On the other hand, it is remarkable how the $\mathrm{NH}_{2}$-terminus, and TM1-TM2 loop decreased their accessibility to the polar agent NiEdda, suggesting that both regions gain depth into the membrane. One of the most important findings is that residues at the TM3 segment, in the open conformation, seem to be exposed to NiEdda, a direct demonstration that the permeation pathway changed its diameter upon asymmetric incorporation of lyso PC. 

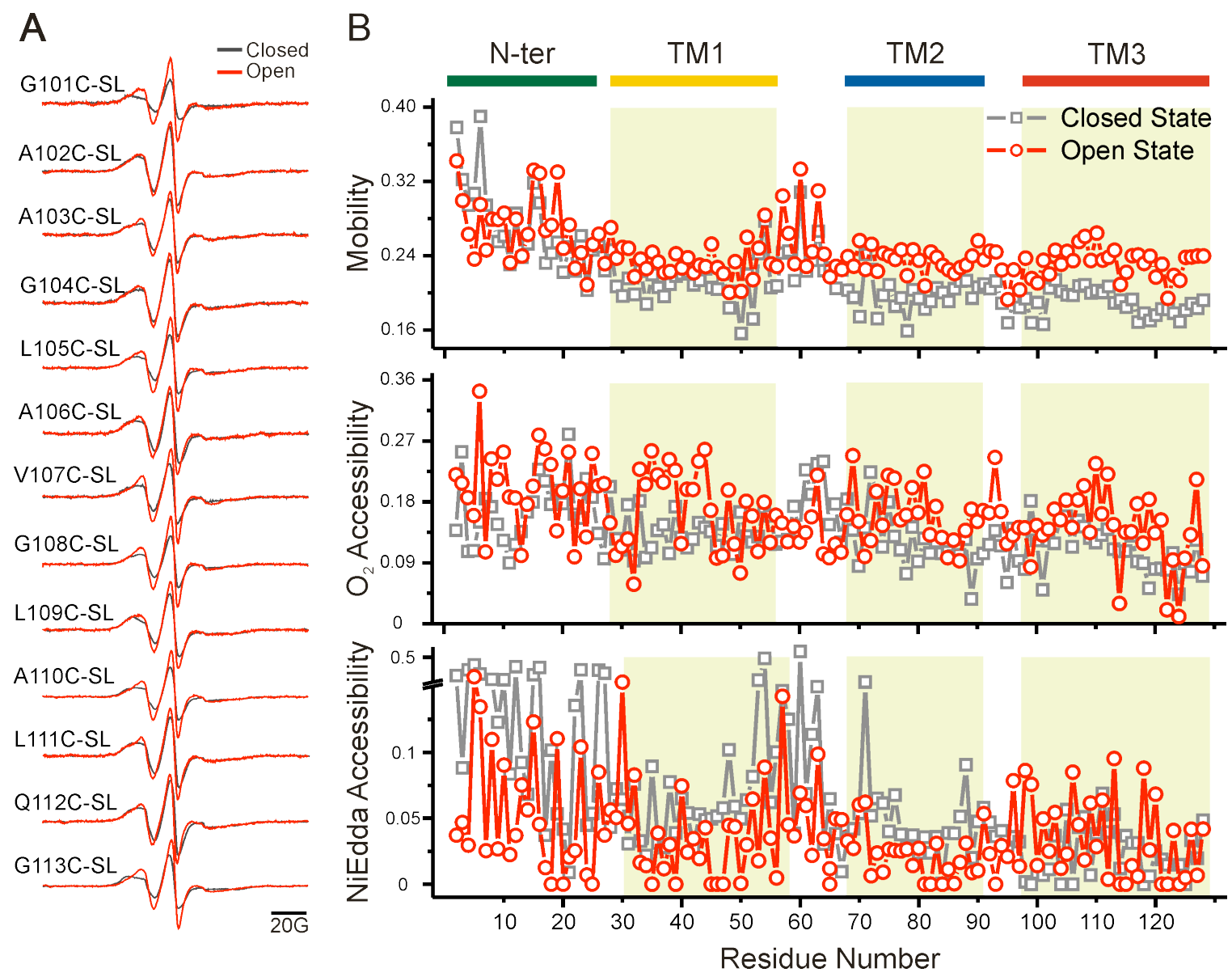

Figure IV. 3. EPR spectroscopy of MscS upon addition of Lyso PC. A) Representative Xband EPR spectra of consecutively spin-labeled mutants from a portion of the permeation pathway (101-113) in TM3. Gray traces were obtained from channels in the closed conformation and red traces represent the open state. All spectra were obtained from samples with the same protein to lipid ratio, and using a dielectric resonator with the microwave power set to $2 \mathrm{~mW}$. Channel opening was obtained in DOPC:POPG $+25 \mathrm{~mol}$ $\%$ LPC vesicles. B) Residue-specific environmental parameter profiles obtained in the open (red) and closed (gray) conformations for the $\mathrm{NH}_{2}$ terminal and $\mathrm{TM}$ segments: mobility parameter $\Delta \mathrm{H}_{\mathrm{o}}^{-1}$ (top), $\mathrm{O}_{2}$ accessibility parameter $\Pi_{2}$ (middle), and NiEdda accessibility parameter $\Pi$ NiEdda (bottom). Yellow areas represent the TM segment assignment derived from $\mathrm{MscS}$ crystal structure. 

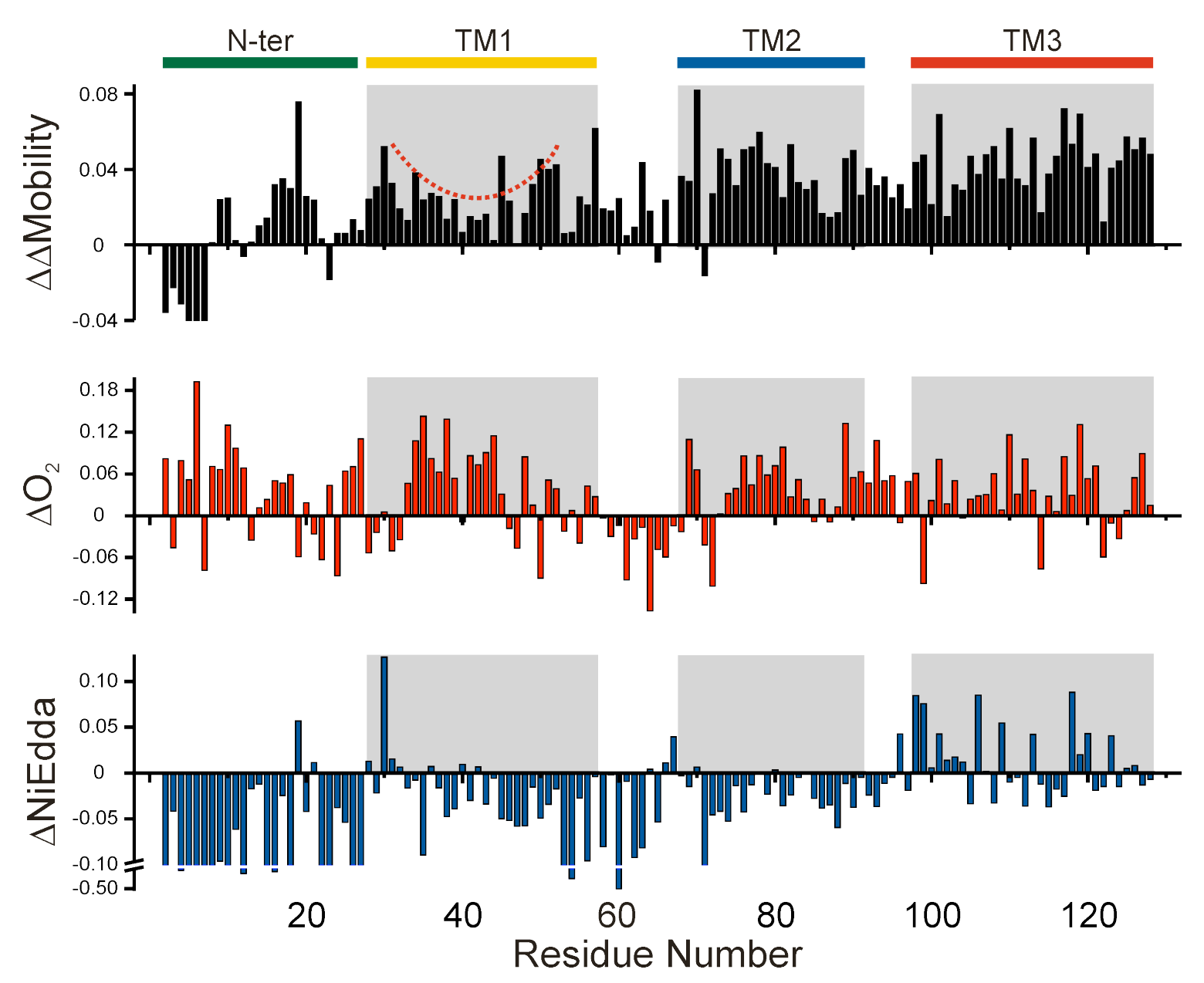

Figure IV.4. Extent of environmental parameter changes of MscS upon addition of Lyso PC. $\Delta$ represents open minus closed state parameters. Top panel, changes in mobility $\left(\Delta \Delta \mathrm{H}_{\mathrm{o}}^{-1}\right)$. The red broken curve pinpoints the decrease in mobility changes towards the middle of TM1. Middle panel, differences in $\mathrm{O}_{2}$ accessibility $\left(\Delta \Pi \mathrm{O}_{2}\right)$. Bottom panel, changes in NiEdda accessibility ( $\Delta \Pi \mathrm{NiEdda})$. Gray areas represent the TM segment assignment derived from MscS crystal structure. 
To better appreciate the EPR results, we have mapped the raw data set (Figure IV.5) and the difference between the open and closed states parameters (Figures IV.6) in the experimentally determined three-dimensional models. Mapping the environmental parameters, onto the closed conformation model and the crystal structure, corroborates that several rearrangements take place in the open conformation. Although the $\mathrm{NH}_{2}$ terminus is still the most dynamic segment on the TM domain (Figure IV.5.A), some of its residues decreased their mobility upon opening (Figure IV.6.A, blue); while a large amount of the segment gained $\mathrm{O}_{2}$ accessibility and lost NiEdda exposure (Figures IV.6.A). We found small dynamic changes along the TM1, in fact the mobility register appears to be the same than in the closed conformation. On the other hand, the TM1 region comprising positions L35C-SL to S49C-SL shows an abrupt increase in $\mathrm{O}_{2}$ accessibility that looks more evident when mapped onto the crystal structure surface (Figures IV.5.B and IV.6.B, red). These accessibilities rearrangements could occur if the $\mathrm{NH}_{2}$-terminus segment moves away from the TM1 upon opening, leaving space for the lipids to interact with this region. We could also notice that, once the channel opens, the beginning of TM1 (positions S26C-SL to V32C-SL) is partially exposed to the aqueous environment (bottom panel of Figures IV.4 and right panel of IV.6), whereas the TM1TM2 loop partially decreases its accessibility to the aqueous membrane interface. 

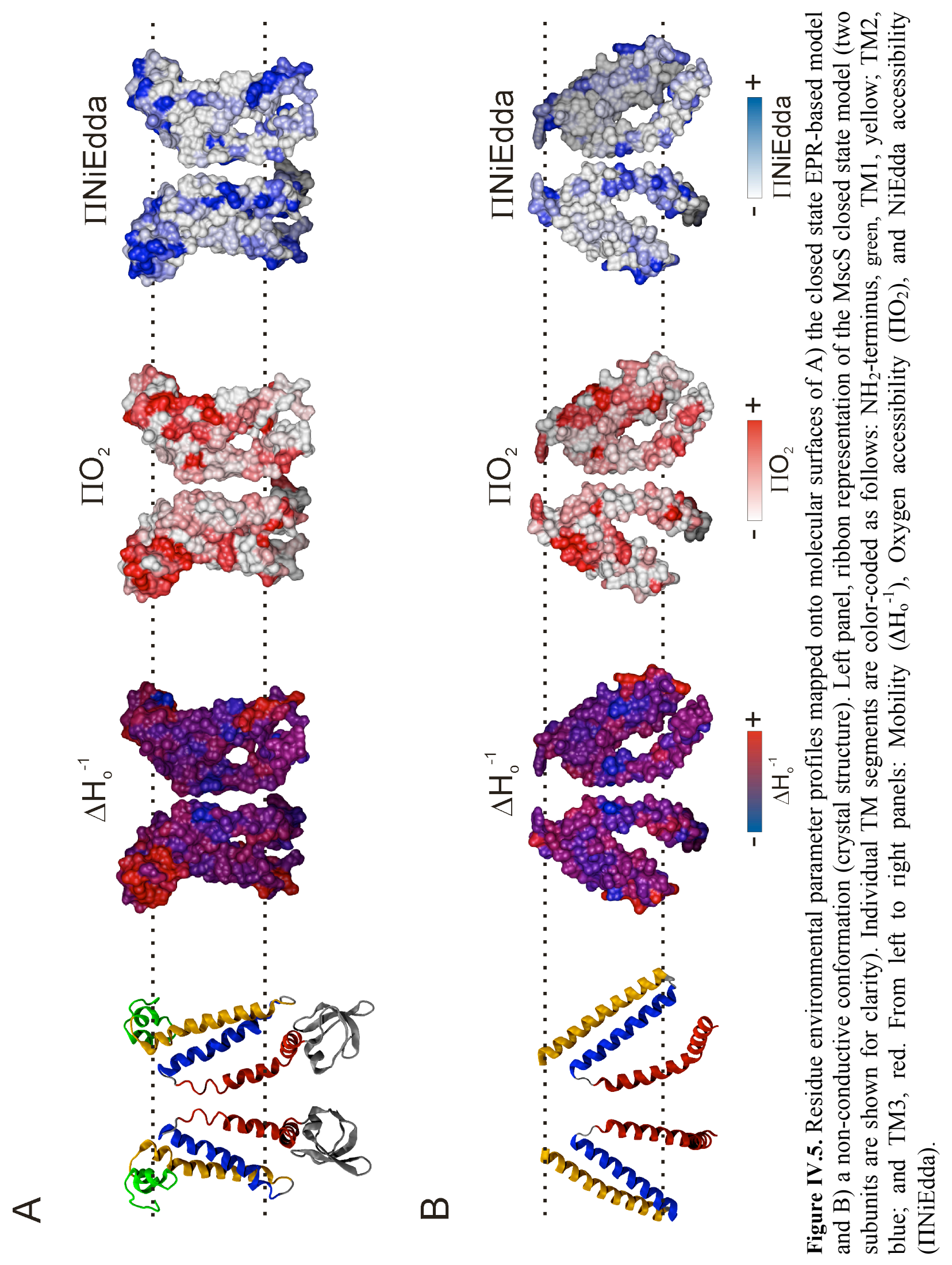

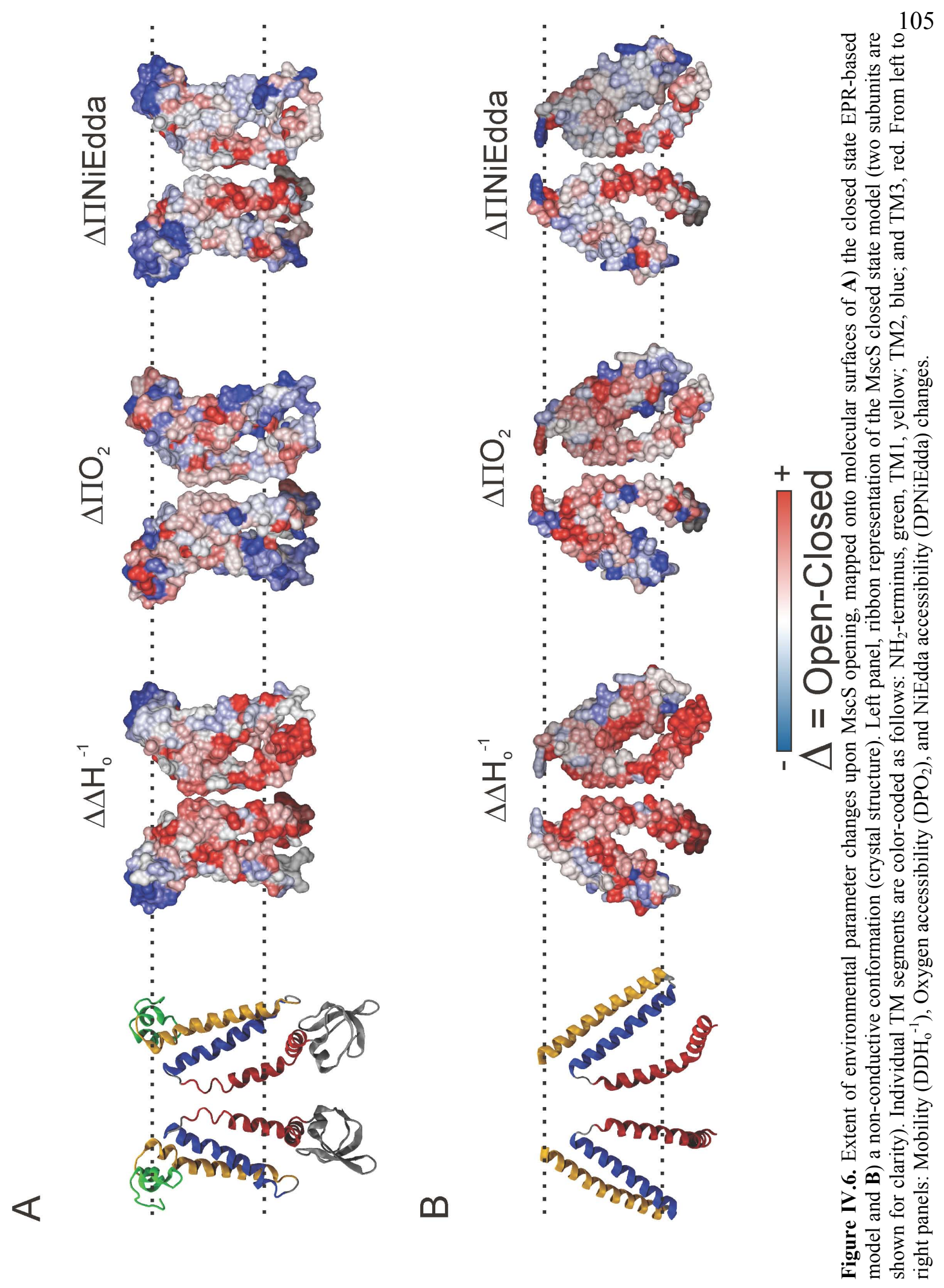
The TM2 undergoes big conformational changes upon lyso PC incorporation.

In the closed conformation the amino-terminal part of TM2 was exposed to the membrane, whereas upon opening the $\mathrm{O}_{2}$ accessibility decreases while the NiEdda increases. On the other hand, the carboxy-terminal region of this segment that hardly had accessibility to $\mathrm{O}_{2}$ in the closed conformation appears more exposed to the membrane once the channel opens (Figure IV.6.B).

Upon addition of lyso PC the TM3 segment becomes more dynamic (Figures IV.5, and IV.6), and even though the $\mathrm{O}_{2}$ accessibility did not change much between the closed and open conformation, there are certain regions of high $\mathrm{O}_{2}$ accessibility (A110CSL to Q112C-SL, and M117C-SL to G121C-SL). According to our results and the crystal structure (Bass et al., 2002), the conduction pathway starts at the TM2-TM3 loop, near to position V96C-SL where the NiEdda accessibility differences are more apparent. The TM3 exposure to NiEdda seems to follow a pattern that is easier to recognize when mapped onto molecular surfaces. The face of the TM3 helix that in the open state is available for NiEdda collision is not pointing towards the permeation pathway when mapped in non-conducting models (right panels of Figures IV.5.A-B and IV.6.A-B), suggesting that the TM3 has to undergo a substantial rotation about its principal axis, in order to satisfy our experimental data.

\section{IV.4.3 Analysis of the Conformational Rearrangements during Activation}

\section{Gating}

We have used a quantitative approach to estimate the secondary structure from our one-dimensional data set, of reconstituted $\mathrm{MscS}$ upon incorporation of lyso PC. Consequently, we would use this assignment to build an EPR-based three-dimensional 
model of MscS in an open conformation. This approach, that has been successfully used before (McHaourab and Perozo, 2002; Perozo et al., 1998; Vasquez et al., 2007b) is based on a Fourier transform method (Cornette et al., 1987) that estimates the periodic behavior of a segment, based on residue specific properties such as the EPR structural parameters (McHaourab and Perozo, 2002).

Figure IV.7 shows the power spectra obtained from MscS TM domain in an open conformation. In the closed state the TM domain, with the exception of the TM3, displayed a prominent peak within the $\alpha$-helical region $\left(80-120^{\circ}\right.$, gray curves on Figure IV.7.A-C). Even though the relative area of the power spectra peaks in the open conformation are wider than in the closed state, the overall periodicity of the $\alpha$-helical structures is preserved. This is a clear indication that there is no general unwinding of the TM domain segments upon opening, and it also suggests that the main movements could occur as rigid bodies, with a few changes in secondary structure. Interestingly, the TM3 segment displays the most profound changes. When the pore is closed, the spin-label probe is restricted in multiple protein contacts (see top panel of Figure IV.3.B); therefore periodicity cannot be distinguished (gray curve on Figure IV.7.D). When the pore diameter increases, as a consequence of channel opening, the spin label has enough motional freedom to report the environment that surrounds it, as Figure IV.7.D shows (red curve, double arrow). This represents another evidence of MscS opening upon asymmetric incorporation of lyso PC. 


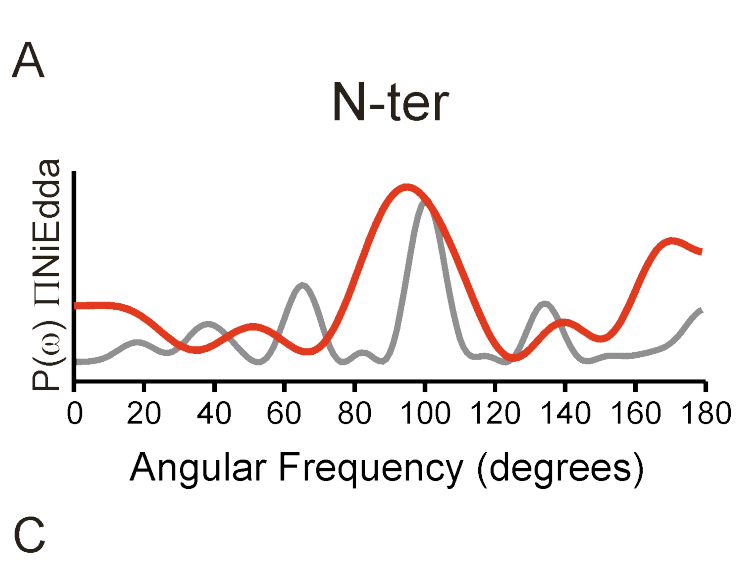

TM2

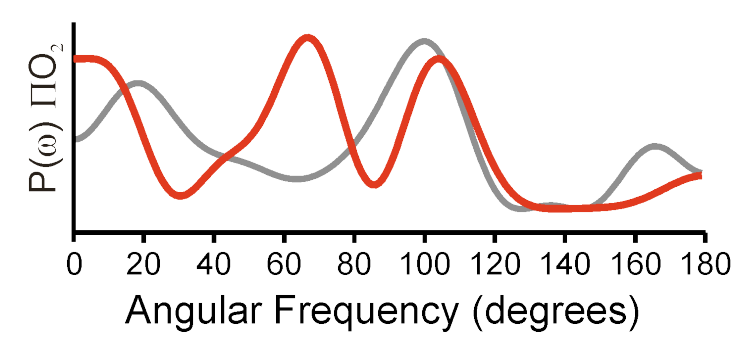

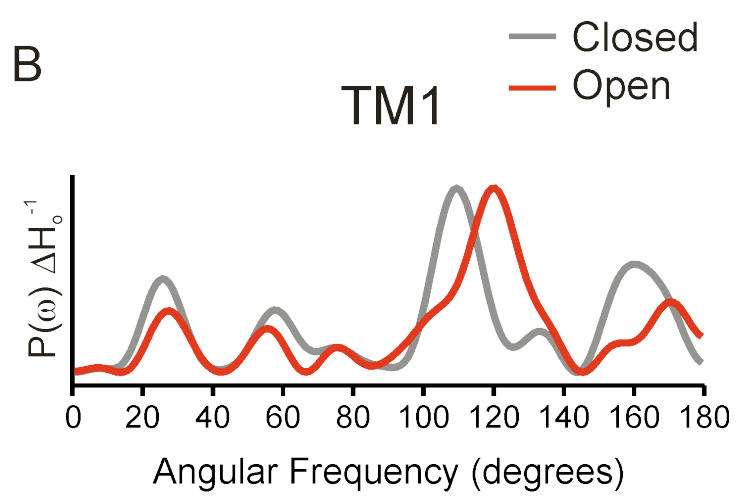

$\mathrm{D}$

TM3

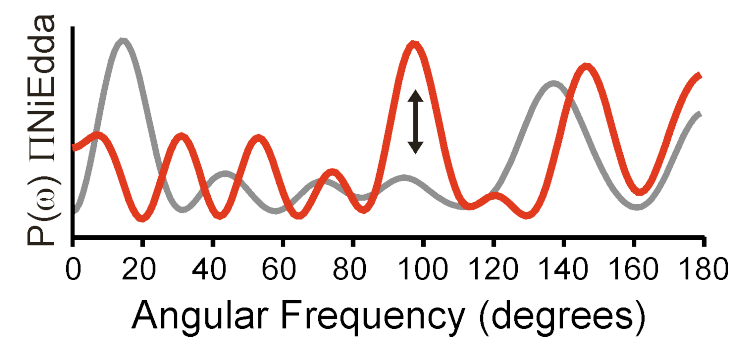

Figure IV.7. Calculation of the Fourier transform power spectra of the accessibility profiles for the transmembrane domain in the closed (gray curves) and open conformations (red curves). The $100^{\circ}$ peak of angular frequency corresponds to an $\alpha-$ helical structure. A) Power spectrum of the $\mathrm{NH}_{2}$-terminal segment $\mathrm{INiEdda}$ profile. B) Power spectrum of the TM1 segment $\Pi_{2}$ profile. C) Power spectrum of the TM2 segment $\Pi_{2}$ profile. D) Power spectrum of the TM3 segment $\Pi$ NiEdda profile. The black arrow shows the periodicity gain of the pore segment upon opening. 
We calculated the periodicity index for each TM segment (Figure IV.8) (Cornette et al., 1987) using a sliding window approach (Rees et al., 1989). With the periodicity index it could be estimated the probable limits of secondary structures within the TM segments, which will serve as the basis for structural assignments. According to the analysis of the accessibility profiles, in this open conformation there is a loss of periodicity at the beginning of the $\mathrm{NH}_{2}$-terminus, instead the helix approximately begins at position V18C-SL up to position A28C-SL (Figure IV.8.A). On the other hand, the TM1 segment seems to keep its periodicity pattern, although more loose, when analyzed on the light of the mobility parameter (Figure IV.8.B). The TM2 segment undergoes major rearrangements upon opening. In the closed state, after position A71C-SL there is a dramatic decrease in $\mathrm{O}_{2}$ accessibility, we attributed this to the fact that the TM2 is in closed proximity to the TM3 in the resting conformation (Vasquez et al., 2007b). After MscS opens the TM2 seems to move away from the TM3, and this conformational change is not only reflected in the increase of mobility and membrane accessibility but also in the gain of $\alpha$-periodicity at the carboxy-terminal end of TM2 (Figure IV.8.C). The TM3 secondary assignment in the open state cannot be described respect to the closed conformation, since this reporter-based approach could not detect its periodicity in the ground state. Nevertheless, with the current $\mathrm{NiEdda}$ and $\mathrm{O}_{2}$ parameter profiles we have determined that the segment that lines the pore is in its majority an $\alpha$-helix, with short loops connecting its secondary structure elements (Figure IV.8.D). 

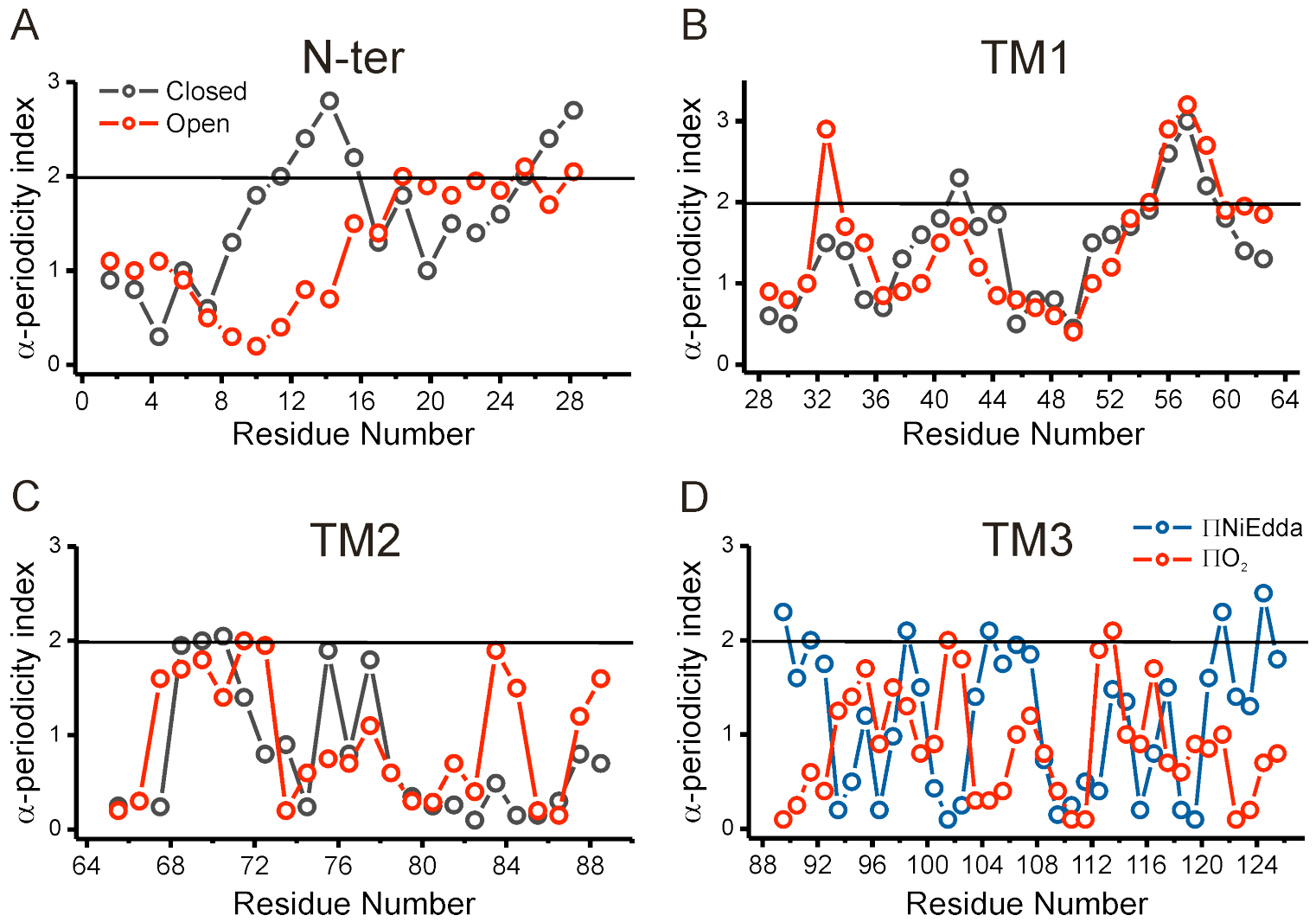

Figure IV.8. Window periodicity analysis for MscS transmembrane domain in the closed and open states. The $\alpha$-helical periodicity index was calculated as described earlier (Cornette et al., 1987) with an angular range from $80^{\circ}-120^{\circ}$ and a sliding window of seven residues. The horizontal line represents the threshold at which the periodicity of the windowed segment is likely $\alpha$-helical. A) Periodicity analysis of the $\mathrm{NH}_{2}$-terminus NiEdda parameter for the closed (gray) and open (red) conformations. B) Periodicity analysis of the $\mathrm{TM} 1 \mathrm{O}_{2}$ parameter for the closed (gray) and open (red) conformations. C) Periodicity analysis of the $\mathrm{TM} 2 \mathrm{O}_{2}$ parameter for the closed (gray) and open (red) conformations. D) Periodicity analysis for the open conformation of the TM3 NiEdda (blue) and $\mathrm{O}_{2}$ parameters (red). 
To further examine the magnitude of the conformational changes upon opening, we have superimposed the individual environmental moments for the spinlabeled residues from the closed and open state, on a helical wheel representation (Figure IV.9). In particular, the $\mathrm{NH}_{2}$-terminus in the resting conformation resides at the membrane interface (Vasquez et al., 2007b), but in the open state the magnitude of the $\mathrm{O}_{2}$ vectors are larger than the ones for NiEdda accessibility (Figure IV.9.A), thus we consider that this segment has to be placed in a more hydrophobic region of the membrane interface. In general, the vector analysis in the context of a helical wheel projection indicated that the conformational changes upon opening could be explained by rotation of the helices, along with tilting. Apart from the TM3, each of the TM segments, including the $\mathrm{NH}_{2}$-terminus, have to rotate about $36-50^{\circ}$ (Figure IV.9.A-C) in order to expose to the membrane those residues with higher $\mathrm{O}_{2}$ accessibilities. On the other hand, the TM3 has to undergo a major rotation $\left(135^{\circ}\right.$, see Figure IV.9.D) to allow those residues with larger exposure to NiEdda face the permeation pathway in the open conformation. 
A

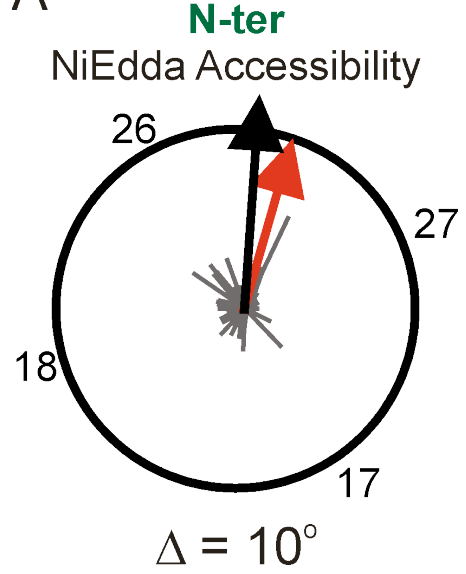

B
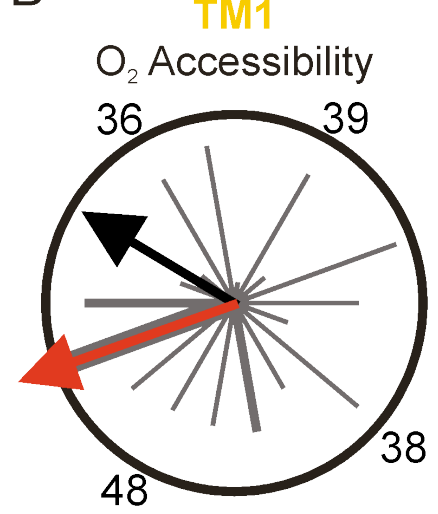

$$
\Delta=50^{\circ}
$$

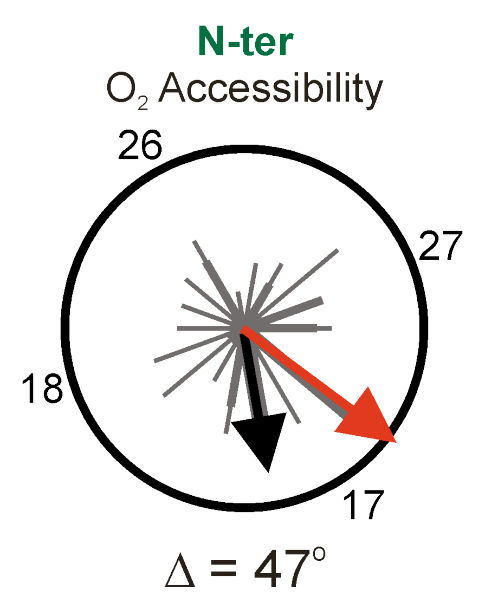

C

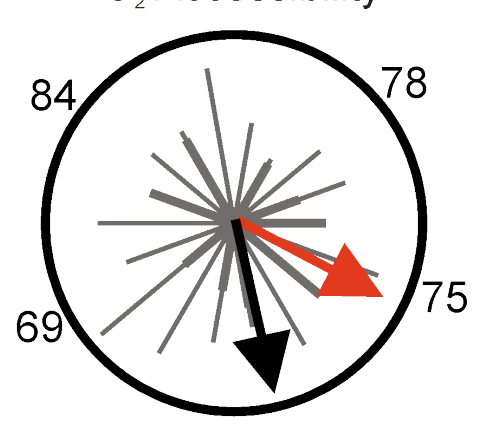

$$
\Delta=36^{\circ}
$$

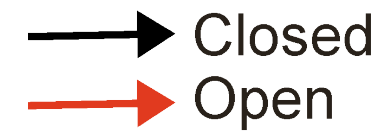

$\mathrm{D}$ NiEdda Accessibility

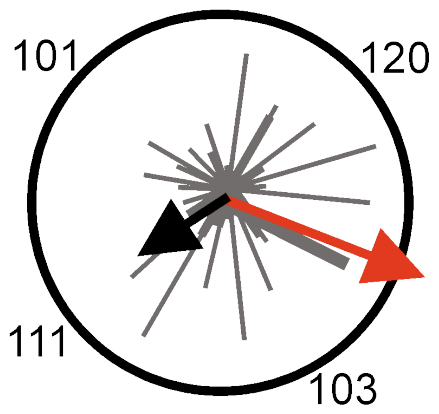

$\Delta=135^{\circ}$

Figure IV.9. Structural analysis of $\mathrm{MscS}$ transmembrane domain in the open conformation. Environmental parameters have been superimposed in a polar coordinate representation. Resultant moments, for the closed (black arrow) and open (red arrow) conformations, were calculated for the accessibilities of each transmembrane segment and superimposed on a helical wheel. A) Helical wheel representation of the $\mathrm{NH}_{2}$-terminus. Resultant vectors were calculated for the NiEdda (left panel) and $\mathrm{O}_{2}$ (right panel) accessibilities. B) Helical wheel representation of the TM1 segment. Resultant vectors were calculated for the $\mathrm{O}_{2}$ accessibility. C) Helical wheel representation of the TM2 segment. Resultant vectors were calculated for the $\mathrm{O}_{2}$ accessibility. D) Helical wheel representation of the TM3 segment. Resultant vectors were calculated for the NiEdda accessibility. 
All of the conformational changes that occur on MscS TM domain upon opening can be summarized (see Figure IV.10) as follows: 1- The $\mathrm{NH}_{2}$-terminus seems to be in a deeper or more hydrophobic region of the lipid bilayer interface, since it displayed

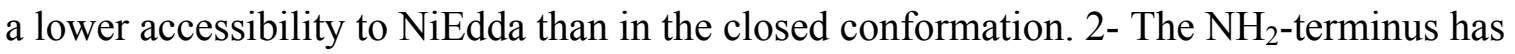
to extend away from the TM1 as the top section of the TM1 gained accessibility to $\mathrm{O}_{2}$. 3The TM1-TM2 loop is not longer fully exposed to the aqueous environment, since its accessibility to NiEdda considerably decreased. 4- The carboxy-terminal region of the TM2 is not longer in contact with the TM3, given the fact that the membrane exposure increased. 5- The pore has to expand its radius to allow NiEdda penetration, along with the rotation of the TM3 helices to place the solvent accessible regions towards the permeation pathway.

Closed State (EPR Model)

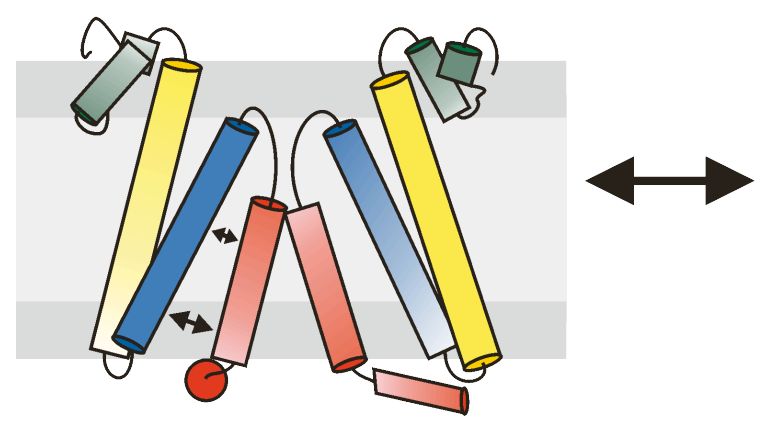

Open State (EPR Model)

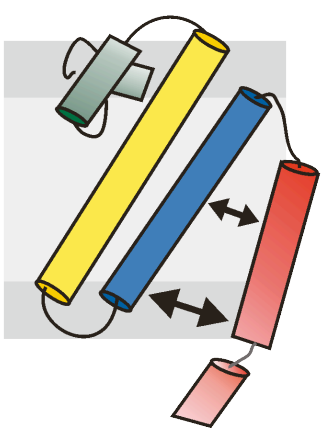

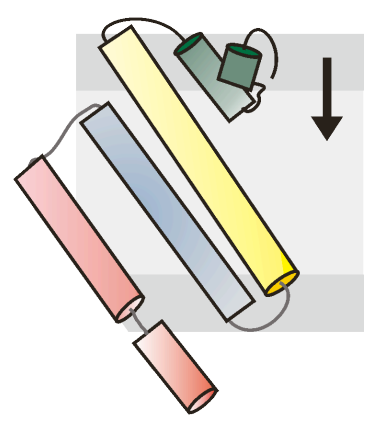

Figure IV.10. Schematic representation of the structural rearrangements underlying gating in $\mathrm{MscS}$. Left panel, cartoon representation of MscS closed state model from EPR based refinement. Individual TM segments are color-coded as follows: $\mathrm{NH}_{2}$ terminus, green; TM1, yellow; TM2, blue; and TM3, red. Right panel, cartoon representation of $\mathrm{MscS}$ in the open conformation according to the EPR experimental data. The arrows show the key rearrangement in the TM segments of MscS upon opening. 


\section{IV.5 DISCUSSION}

Lysophosphatidylcholine has been used as a biophysical tool to modulate the gating of several mechanosensitive and stretch ion channels, even though it has been difficult to predict what kind of physiological stimulus lyso PC is mimicking (Lundbaek and Andersen, 1994; Maingret et al., 2000; Martinac et al., 1990; Perozo et al., 2002a; Perozo et al., 2002b). The activation of mechanosensitive channels through this coneshaped lipid occurs by torque generation in the membrane due to its asymmetric distribution in the two membrane leaflets (Markin and Sachs, 2007). The local change of membrane spontaneous curvature induces a rearrangement of the protein shape that will lead to mechanosensitive channel activation, as previously determined for MscL (Perozo et al., 2002a). We have used the same approach to activate MscS, in order to trap it in an open conformation.

The extent of MscS conformational changes were evaluated through patch-clamp experiments (Figure IV.1), where the lateral pressure profile was modified by the incorporation of lyso PC into the internal leaflet of the bilayer (bath). Lyso PC incorporation seems to provide enough energy to trigger $\mathrm{MscS}$ gating, and more important the signature inactivation process of $\mathrm{MscS}$ was completely absent during this type of activation protocols. Although, we have not systematically characterized the incorporation of lyso PC in the outer leaflet (pipette), we consider that MscS activation would not be different if activated by the periplasmic side. Since the location of residues involved with tension sensitivity are located at the membrane interface of both periplasmic and cytoplasmic faces (Nomura et al., 2006). 
As we were doing these experiments an important question emerged. Why MscS does not feature a time-dependent inactivation process upon asymmetric adsorption of lyso PC? For instance, MscL does not inactivate, and the tension needed to open it is approximately twice the one required to activate MscS (Figure IV.11). The tension interval where $\mathrm{MscS}$ activity occurs seems to allow further conformational rearrangements from open to inactivated/desensitized states, such changes are absent in MscL. Besides, it is well known that the time constant of inactivation in MscS depends on the rate of the pressure applied; therefore the channel would only visit the closed and open state under saturating stimulus (Akitake et al., 2007a; Akitake et al., 2005). For these reasons, we propose that lyso $\mathrm{PC}$ activation resembles the opening of $\mathrm{MscS}$ by saturating pressure pulses where the open state of the channel would be favored. In addition to this plausible explanation, $\mathrm{MscK}$, a channel with similar conductance that opens in the same tension interval than $\mathrm{MscS}$, is rarely observed in spheroplasts preparations $(70 \%$ less than $\mathrm{MscL})$ while featuring long mean closed times ( $\mathrm{Li}$ et al., 2002), this single channel behavior could be attributed to the presence of a not yet characterized inactivation process in MscK. Regardless of the effect that lyso PC is causing in the membrane, we have found a biophysical tool that allowed us to trapped MscS in the open state in order to obtain spectroscopical data under steady state conditions. 

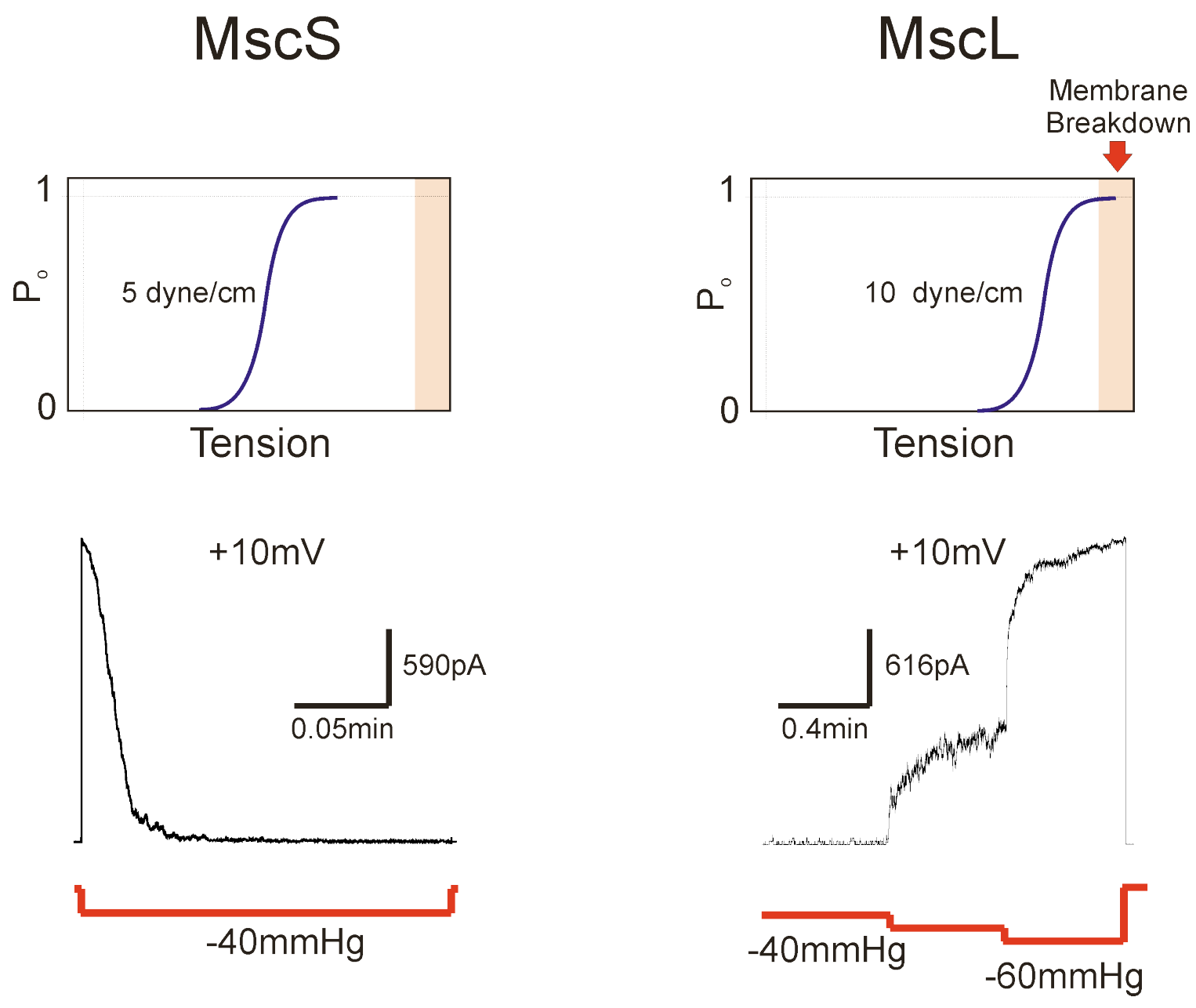

Figure IV.11. Functional differences between $\mathrm{MscS}$ and MscL. Top panel illustrates the activation threshold of $\mathrm{MscS}$ and $\mathrm{MscL}$, respectively. Bottom panel shows macroscopic currents activated by pressure pulses, revealing the presence of a timedependent inactivation process in MscS that is totally absent in MscL. 
The incorporation of lyso PC into the channel containing vesicles was accompanied by a large increase in probe mobility in most regions of the TM segments, with the exception of the $\mathrm{NH}_{2}$-terminus and the central part of the TM1. Even though the $\mathrm{NH}_{2}$-terminus became less mobile upon gating, in the open state it is still the most dynamic and most solvent exposed segment of the TM domain; therefore it is in the periphery (away from the channel central axis of symmetry), like in the closed conformation (Vasquez et al., 2007b). For the TM1 in particular the dynamic alterations were not uniform, given the fact that the largest changes were observed at both ends of the TM1 helix (residues V29C-SL to L35C-SL, and S49C-SL to I57C-SL, see broken red dotted curve on Figure IV.4). Nomura and collaborators performed a hydrophilic scan along the TM1 and TM2 segments in order to modify the interactions between the channel and the lipids, through a redistribution of the lateral pressure profile (Nomura et al., 2006). Using this approach, they found that asparagine substitution of residues located at the membrane interface (periplasmic and cytoplasmic ends of both helices) resulted in loss of function phenotypes (A51N/F68N and I37N/L86N), since the mutants were insensitive to lateral tension modifications. Combining the outcomes of this functional information with our EPR results make us suggest that the major conformational changes in the TM1 helix, which faces the membrane, occur on those residues that are nearby the tension sensor, located at both ends of the membrane interface.

According to our solvent accessibility results, the transition from closed to the open state involves substantial rearrangements of the TM domain. When looking at the patterns of accessibilities mapped onto the closed state model and the crystal structure, 
we notice that the data seems to better match the dispositions of the TM1 and TM2 helices in the crystal than in the closed state (Figures IV.5 and IV.6). This conclusion is somehow expected, if we think of the crystal structure as an intermediate conformation towards the open state (Anishkin and Sukharev, 2004; Sotomayor and Schulten, 2004; Sotomayor et al., 2006; Sotomayor et al., 2007). In the open conformation the TM1 and TM2 segments do not lose their helix integrity, but they definitely change the local environment that was previously surrounding them in the closed conformation. The TM1TM2 loop is no longer at the aqueous phase of the bilayer and the TM2 is now asymmetrically solvated with one region of the helix facing TM1 while lipids surround the opposing surface. Tilting and rotation of the TM1 and TM2 helices away from the channel core as rigid bodies would account for these environmental differences between the closed and open conformations, and would further imply an increment in the crosssectional area of MscS TM domain. Since we do not have any other experimental reference on the structure and location of the $\mathrm{NH}_{2}$-terminus, more than the EPR-based closed state model (Vasquez et al., 2007b), we can only predict that during gating this segment loses its accessibility to the polar milieu and moves away from the periphery of the channel symmetry, further exposing the amino-region of the TM1 to the fatty acid chains of the bilayer.

The TM3 segment is highly dynamic in the open state since all of its spin-labeled residues become more mobile after lyso PC incorporation (Figures IV.3 and IV.4), as the pore helices have lost protein-protein contact upon opening. Furthermore, the increase in accessibility to the polar compound NiEdda supporting a water-filled pore contributes to the proposal that we have trapped $\mathrm{MscS}$ in an open conformation. The increase in 
accessibility along the pore helix is not homogeneous, as there are some areas with less accessibility to NiEdda, which instead display high accessibility to the membrane. It is also notable how some positions that outlined a hydrophobic plug in the closed state (Anishkin and Sukharev, 2004; Sotomayor and Schulten, 2004; Sotomayor et al., 2007; Spronk et al., 2006; Vasquez et al., 2007b), in the open conformation exhibit accessibility to the polar milieu, such as A103C-SL, A106C-S1, V107C-SL, L109C-SL, L111C-SL, and G113C-SL.

It is outstanding how the carboxy-terminal part of the TM3 (also called TM3b, 114-128) seems to be also facing the permeation pathway. This TM3 accessibility change would require straightening of the helices, since this segment, after the G113 kink is buried in the closed conformation. These experimental evidences agree with previous molecular dynamic simulations, where the wider conformations predicted would exhibit straighter pore helices (Sotomayor et al., 2006; Sotomayor et al., 2007), as well as with an experimental study where favoring helical formation by G113A prevented inactivation and by G121A prevented inactivation and closure (Akitake et al., 2007a).

In order to satisfy our NiEdda accessibility data, the TM3 helices not only have to expand during gating but also have to rotate approximately $135^{\circ}$, to expose those helix surfaces to the permeation pathway that in the closed state were involved in inter-subunit contacts. This rotation would break the hydrophobic seal, as it was suggested in an earlier study where the pore residues were systematically mutated, to larger and more hydrophobic residues, increasing the pressure required for gating (Edwards et al., 2005). These results could imply that given the non-polar nature of the spin-label, some of the pore labeled residues might be more difficult to open, thus not all of the probes would be 
sampling the same open conformation. This hypothesis has to be further tested using bulk assays, like the one used for MscL (Kocer-Sagiroglu et al., 2005; Kocer et al., 2005), upon incorporation of lyso PC with samples labeled in the TM3 segment. In any instance, we would not expect to find higher magnitudes of NiEdda accessibilities than the ones already found, since the diameter of NiEdda is too large (8-9 $\AA)$, in addition to the nitroxide length $(\sim 5 \AA)$, to be freely diffusing in a pore that has been predicted to be in the range of 13-17 $\AA$ (Akitake et al., 2007a; Sotomayor et al., 2007).

Trapping MscS in an open state, by the asymmetric incorporation of lyso PC into DOPC:POPG vesicles, let us obtained a fairly extensive collection of constraints (128 spin-labeled positions) that are currently being used to derive a three-dimensional model of MscS in the open conformation. Schematic representations of MscS are shown in Figure IV.10, which recapitulate all the conformational changes that have to occur according to our EPR constraints during gating. In summary, the $\mathrm{NH}_{2}$-terminus inserts into the membrane and moves away from the top part of the TM1, the TM1-TM2 hairpin tilts and rotates so the TM2 is no longer in contact with the TM3, and finally the pore increases its radius accompanied by the rotation of the TM3 helices towards the permeation pathway. The available data is limited to changes in local environmental parameters. Nevertheless, combining an efficient computational method with the experimental accessibility data will allow us to better understand the type, direction, and magnitude of the conformational changes that occur in the transmembrane domain of MscS upon opening, including the $\mathrm{NH}_{2}$-terminus segment. 


\section{CHAPTER V: Understanding the Molecular Basis of the Voltage-Modulation in}

\section{MscS}

\section{V.1 ABSTRACT}

The mechanosensitive channel of small conductance $(\mathrm{MscS})$ is a membrane protein thought to act as a safety valve in bacteria, regulating the release of ions and small solutes when gated by changes in membrane tension, under challenging osmotic conditions. The influence of voltage on channel inactivation and the functional state depicted by the available crystal structure of MscS remain unresolved. Snapshot of the channel in the crystal structure has shown the presence of lipid-facing charged residues in the transmembrane (TM) domain, and molecular dynamics simulations have pointed out salt bridges between the TM connecting loop and the cytoplasmic basket. These residues and interactions are the prime candidates responsible for the voltage sensing process. In an effort to relate electrophysiological measurements and conformation of the crystal, we report here the response of $\mathrm{MscS}$ to voltage and pressure as determined by patch-clamp and evaluated further by molecular dynamics simulations. Electrophysiological experiments revealed that $\mathrm{MscS}$ is a slightly anion-selective channel with a conductance of $\sim 1 \mathrm{~ns}$, activated by pressure and inactivated in a voltage-dependent manner. And simulations showed that $\mathrm{MscS}$ restrained to the crystal conformation exhibits low conductance, mainly from $\mathrm{Cl}^{-}$ions. Neutralization of TM charged residues does not affect MscS ability to protect against sudden osmotic changes, and does not eliminate the voltage dependence of inactivation. In addition, disruption of the salt bridge interaction between the TM domain and the cytoplasmic basket does not affect voltage dependence, but interestingly generates loss of function phenotypes. Even though our results still do 
not reveal the location of a voltage sensor, more studies are underway in order to understand the voltage-dependence of the inactivation process in MscS. 


\section{V.2 INTRODUCTION}

Bacterial cells depend on mechanosensitive channels to protect themselves from sudden changes in osmotic pressure and to prevent cell lysis (Blount et al., 1997; Hamill and Martinac, 2001; Levina et al., 1999; Martinac et al., 1987; Perozo, 2006). When subject to osmotic downshock, these membrane proteins act as large and non-selective pores that allow passage of ions and small solutes, thereby alleviating the otherwise lethal osmotic imbalance (Schleyer et al., 1993; Wood, 1999).

Two different mechanosensitive channels have been crystallized, the mechanosensitive channel of large conductance MscL (Chang et al., 1998; Steinbacher et al., 2007; Sukharev et al., 1994), and the mechanosensitive channel of small conductance MscS (Bass et al., 2002; Levina et al., 1999; Steinbacher et al., 2007). Patch-clamp experiments have shown that these proteins are activated by tension in the cell membrane and likely undergo large conformational rearrangements upon gating (Akitake et al., 2005; Berrier et al., 1989; Edwards et al., 2005; Martinac et al., 1987; Sukharev, 2002). Functional assays on knockout E. coli (lacking native MscL, MscS, and MscK) have shown that upon IPTG induction of MscL or MscS, cells can survive when subject to osmotic downshock (Levina et al., 1999), indicating a possible redundancy of function (Batiza et al., 2002).

Although the gating mechanism of MscL seems to be a rather "simple" process and has been extensively studied through experiments and theoretical modeling (Betanzos et al., 2002; Colombo et al., 2003; Gullingsrud et al., 2001; Gullingsrud and Schulten, 2003; Kong et al., 2002; Perozo et al., 2002a; Perozo et al., 2002b; Sukharev et al., 2001b), the gating of MscS remains largely undetermined at the molecular level. 
MscS gating is a complex process that involves an activation mechanism that strongly depends on the rate of applied pressure and an inactivation process modulated by voltage (Akitake et al., 2005; Sotomayor et al., 2007).

The quest to understand how $\mathrm{MscS}$ works has greatly benefited from the availability of its x-ray crystal structure, which has served as reference to interpret experimental results. The structure, which is solved at $3.7 \AA$ resolution and recently further refined (Bass et al., 2003; Steinbacher et al., 2007), revealed a homoheptamer featuring three TM helices (labeled TM1, TM2, and TM3A-TM3B) and a large cytoplasmic domain with seven side openings and one distal cytoplasmic entrance (Figure V.1.A). Molecular dynamics simulations have suggested that the crystal structure likely represents an inactive or intermediate state, since the crystal conformation exhibits low conductance and high anionic selectivity (Anishkin and Sukharev, 2004; Sotomayor and Schulten, 2004; Sotomayor et al., 2006) along with dewetting transitions observed throughout the TM pore (Anishkin and Sukharev, 2004; Sotomayor and Schulten, 2004; Spronk et al., 2006). The simulations also revealed an asymmetric closure of MscS when equilibrated in a relaxed membrane (Sotomayor and Schulten, 2004; Spronk et al., 2006) and the formation of salt-bridges between conserved residues (Asp 62 and Arg128, see Figure V.1.B) of adjacent subunits, which might be relevant for MscS gating (Sotomayor and Schulten, 2004). 
A

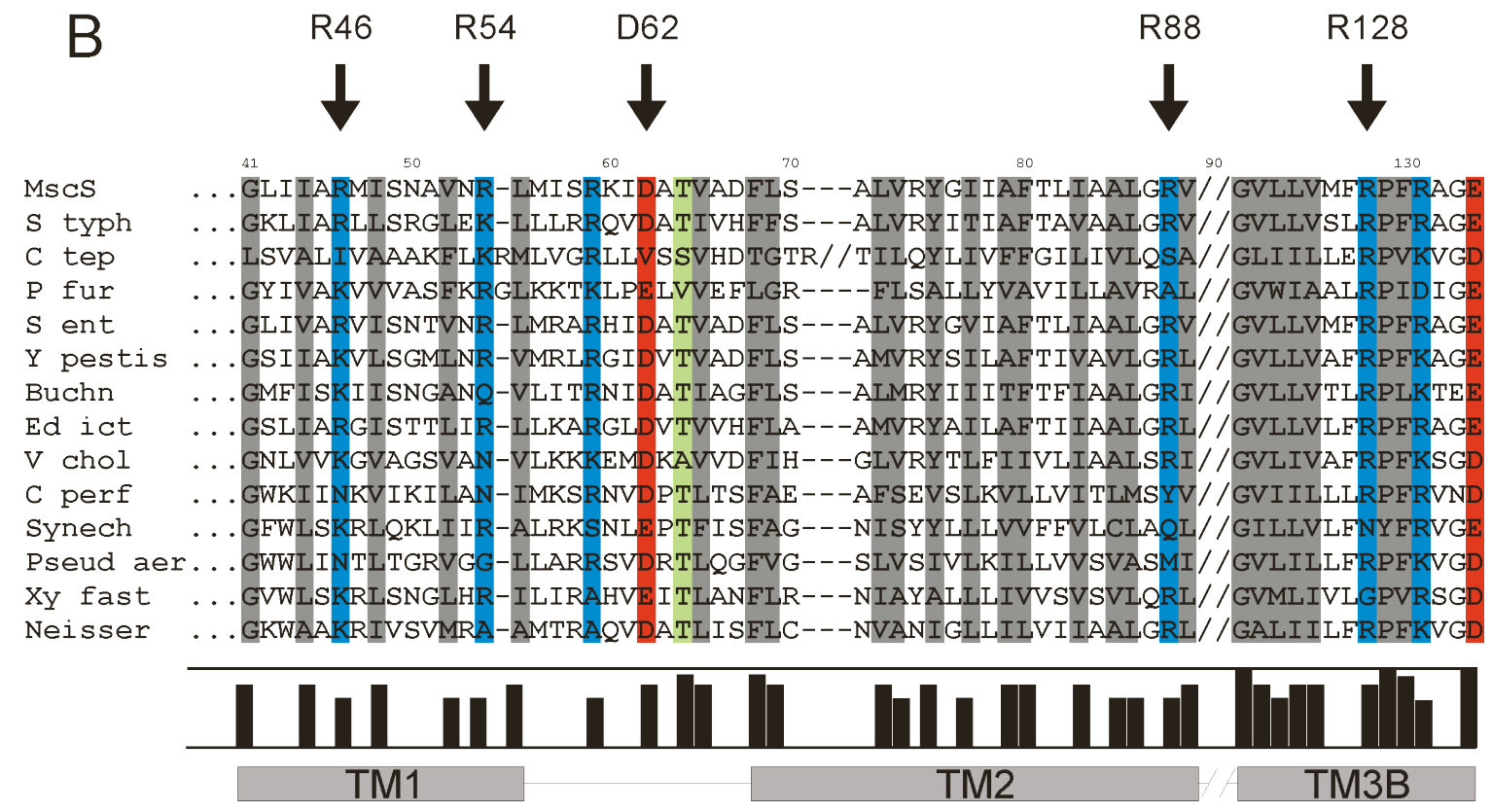

Figure V.1. Architecture of MscS and Sequence Alignment of its Transmembrane Domain. A) The crystal structure is shown as a transparent surface with two (out of seven) subunits depicted in ribbon representation. Charged residues (R46 and R54) located on the TM1, (R59, K60, and D62) at the TM1-TM2-loop, (R74 and R88) on the TM2, and (R128 and R131) at the end of TM3B are shown in space-filling representation. B) Sequence alignment of MscS homologs (Bass et al., 2002), showing the positive and negatively charged residues along the transmembrane domain. 
The present chapter is aimed at establishing a clear correlation between the crystal structure, its functional state, and the role of individual amino acids in gating and ion permeation. Site directed mutagenesis and functional assays allow the replacement of specific amino acids throughout the protein sequence and monitor the effect on its expression, cell growth, and its ability to respond to osmotic challenges. These effects could show the relevance of the site being mutated and indirectly probe its participation in gating. Several studies have been performed on specific mutants of MscS that lead to gain of function or loss of function phenotypes (GOF and LOF, respectively) (Miller et al., 2003b), as well as changes in the electrophysiological response of the channel (Edwards et al., 2005; Nomura et al., 2006, 2007). In general, GOF mutations are defined as those that favor opening of the channel, thereby causing leakage of solutes and growth suppression. On the other hand, LOF mutations impair the ability of MscS to rescue the cell when subject to osmotic downshock.

Initially, we carefully characterized the experimental and simulated $\mathrm{MscS}$ ion conduction, the voltage-dependence of the inactivation of WT MscS under patch-clamp conditions, and its ability to respond in vivo to changes in osmotic conditions. Subsequently, we carried out multiple functional assays on selected mutants. The mutations were performed at positions of charged residues on TM1, TM2 and the TM1TM2 loop, as well as residues putatively involved in inter-subunit salt-bridge formation (Figure V.1.A), observed throughout simulations (Sotomayor and Schulten, 2004). The salt-bridge candidates are located at the TM1-TM2 connecting loop and at the end of TM3B, and their role on MscS function is not evident either from MscS structure or from its sequence. If the crystal structure is depicting a functionally relevant conformation, and 
the dynamics described by MD simulations is similar to that of the native and functional protein, mutations at these sites should perturb the response of MscS. Indeed, we report here multiple LOF phenotypes for MscS mutants in which the charged nature of wild-type residues (conserved residues, see Figure V.1.B), putatively involved in the salt bridge, is modified. In addition, EPR spectroscopy experiments indicate that the formation of the above mentioned salt-bridges might occur in the open or inactive state, but not in the closed one. The effect of these substitutions on the salt-bridge does not seem to affect voltage dependence, instead seems to mediate the communication between the TM domain and the cytoplasmic basket.

Unfortunately, we have not been able to solve the uncertainty surrounding the voltage-dependence phenomenon. In this chapter, I will compare and discuss our findings in the light of some recently published results in order to establish which residues are relevant in $\mathrm{MscS}$ function regardless of its role on voltage dependence. 


\section{V.3 EXPERIMENTAL PROCEDURES}

\section{V.3.1 Constructs, Strains, and Mutagenesis}

For patch-clamp experiments, MscS was cloned in a vector containing an RGSHis6 epitope at the N-terminus (pQE32) and expressed by IPTG induction in an E. coli strain that lacks MscL, MscS, and MscK (MJF465), i.e., provides a genetically clean background. The E. coli strains MJF453 (containing genomic MscS) and MJF429 (containing genomic $\mathrm{MscL}$ ) were used as well for patch-clamp experiments and purification, respectively. No significant differences were found while using MscS expressed from the genome or from the plasmid. These strains were kindly donated by I. R. Booth (University of Aberdeen).

Mutants were generated for charged residues in the TM1, TM2, TM1-TM2-loop and TM3B of MscS (Figure V.1.A and B). Mutagenesis was performed using the QuickChange ${ }^{\mathrm{TM}}$ Site-Directed Mutagenesis Kit (Stratagene).

\section{V.3.2 Spheroplast Patch-Clamp}

Channel activity was recorded by giant spheroplast patch-clamp following the method described by Martinac and collaborators (Martinac et al., 1987). Patch-clamp measurements were done in the inside-out configuration under symmetrical conditions (200 $\mathrm{mM} \mathrm{KCl}, 90 \mathrm{mM} \mathrm{MgCl} 2,10 \mathrm{mM} \mathrm{CaCl}$, and $5 \mathrm{mM} \mathrm{HEPES}$ ) or asymmetrical conditions $(100 \mathrm{mMKCl}$ in the pipette $/ 300 \mathrm{mMKCl}$ in the bath, all other salts as above), at pH 6 and room temperature. For both conditions, the bath solution contained $300 \mathrm{mM}$ sucrose to keep spheroplasts intact.

Pipettes were made of glass capillaries (Sigma, St. Louis, MO: catalog No. P1174) and were fire-polished until resistance between 2 and $2.5 \mathrm{M} \Omega$ was reached. 
Negative pressure on the patch was obtained by applying suction through a syringe and monitored with a home-made piezoelectric pressure transducer. Single-channel and macroscopic currents were sampled at $10 \mathrm{KHz}$ with an analog filter set to $2 \mathrm{KHz}$. Single channel analyses were carried out using pCLAMP9 (Axon Instruments, Foster City, CA). Macroscopic currents were measured using a two-pulse voltage protocol (an initial hyperpolarizing pulse to $-65 \mathrm{mV}$ was followed by a set of depolarization pulses up to 60 $\mathrm{mV}$ ) and at constant negative pressure. The two-pulse voltage protocol reduced the variability in current amplitude due to changes between pressure and voltage pulses and was performed on the same patch with a 3-minute interval between sets of pulses. Selectivity of MscS was determined using the experimentally measured reversal potential and the Goldman-Hodgkin-Katz (GHK) equation (Hille, 2001)

$$
E_{m}=R T / F \ln \left(P_{\mathrm{K}}^{+}\left[\mathrm{K}^{+}\right]_{\text {out }}+P_{\mathrm{Cl}^{-}}^{-}\left[\mathrm{Cl}^{-}\right]_{\text {in }} / P_{\mathrm{K}}^{+}\left[\mathrm{K}^{+}\right]_{\text {in }}+P_{\mathrm{Cl}^{-}}^{-}\left[\mathrm{Cl}^{-}\right]_{\text {out }}\right) \text {, }
$$

where $E_{m}$ is the membrane potential, $R$ is the ideal gas constant, $T$ is temperature, $F$ is Faraday's constant, $P_{\text {ion }}$ is the ion permeability, [ion $]_{\text {out }}$ is the extracellular ion concentration, and $[\text { ion }]_{\text {in }}$ is the intracellular concentration. The GHK equation used assumes that ions cross the channel independently and the potential drops linearly across the translocation pathway. Hence, the calculated selectivity is only an estimate.

The experimental nominal open probability $\left(N P_{o}\right)$ was determined using

$$
I=N P_{o} i
$$

where $I$ is the macroscopic current, $N$ is the number of identical channels, $P_{o}$ is the channel open probability, and $i$ is the single-channel current at a given membrane potential. 


\section{V.3.3 Molecular Dynamics Simulations}

All the Molecular Dynamics Simulations in this chapter, and its respective analysis were done in collaboration with Marcos Sotomayor from Klaus Schulten Laboratory (University of Illinois at Urbana-Champaign).

Molecular dynamics simulations were performed on a system containing the entire crystal structure of MscS (Protein Data Bank code 1MXM (Bass et al., 2002)) embedded in a membrane bilayer formed by 299 POPC lipids solvated in $>50,000$ explicit water molecules and $200 \mathrm{mM}$ of $\mathrm{KCl}$ (altogether $\sim 224,000$ atom system). Two different starting conformations were assumed. The first conformation was obtained after $\sim 4.5 \mathrm{~ns}$ of dynamics with backbone atoms restrained to their positions in the crystal. The second conformation was obtained after $\sim 5 \mathrm{~ns}$ of molecular dynamics simulation in which opening was induced by forces applied radially in the $\mathrm{x}, \mathrm{y}-\mathrm{plane}$ to $\mathrm{C} \alpha$ atoms of residues 96-113 (corresponding to TM3). In both conformations, all Asp, Glu, Lys, and Arg residues were assumed to be charged, as expected from $\mathrm{pK}_{\mathrm{a}}$ calculations. Details of the corresponding molecular dynamics simulations leading to the baseline conformations can be found in (Sotomayor and Schulten, 2004; Sotomayor et al., 2006).

Molecular dynamics simulations were performed using NAMD 2.5 (Phillips et al., 2005) with the CHARMM27 force field for proteins and lipids (MacKerell et al., 1998), and the TIP3P model for water (Jorgensen et al., 1983). Ionic currents through the MscS TM pore were computed by performing a linear fit to the number of crossing events plotted versus time. Conductances $(g)$ were estimated using $g=\mathrm{I} / \mathrm{V}$, where $\mathrm{V}$ is the in silico applied membrane potential. 


\section{V.3.4 Functional assays}

Downshock assays were developed as previously described (Batiza et al., 2002), with the following modifications: cultures growing in LB plus $500 \mathrm{mM} \mathrm{NaCl}$ were induced at $\mathrm{OD}_{600}$ of 0.6 with $1 \mathrm{mM}$ IPTG; after 1 hour of induction, cells were adjusted to an $\mathrm{OD}_{600}$ of 1.0, pelleted down and then resuspended in $50 \mu \mathrm{l}$ of fresh LB plus $500 \mathrm{mM}$ $\mathrm{NaCl}$. The downshock was made by diluting the cells 1:40 in various shock media ranging from 0 to $500 \mathrm{mM} \mathrm{NaCl}(\mathrm{pH} 7)$. After 5 minutes, cells were serially diluted and 5 $\mu \mathrm{L}$ aliquots were plated on LB agar. Plated cells were grown over night, and the colonies were counted and normalized against the $\mathrm{OD}_{600}$ (measured right before the downshock).

\section{V.3.5 Expression, Purification, and Gel Filtration Analysis}

WT and Mutant channels were expressed and purified as previously described in Chapter II (Vasquez et al., 2007a) using a pQE32 vector containing MscS with His6 epitope at the $\mathrm{NH}_{2}$-terminus. The mutants were expressed in E. coli MJF465 (triple knockout) and in MJF429 ( $\Delta m s c s)$, for oligomeric stability analysis and for EPR experiments, respectively. To evaluate the oligomeric state of the mutants, after

purification samples were run on a Superdex ${ }^{\mathrm{TM}} 200 \mathrm{HR}$ 10/30 (Amersham) and monitored using AKTA FPLC system.

\section{V.3.6 MscS Reconstitution}

After purification, cysteine labeled mutants were reconstituted in pre-formed liposomes following protocol previously described, see details in chapter II and (Vasquez et al., 2007a). For CW-EPR experiments, the reconstitution ratio was 1:750 (mol to mol) protein:lipids. Lipids were resuspended in PBS, $\mathrm{pH}$ 7.5. The dilution method (to decrease the DDM concentration below its $\mathrm{CMC}$ ), in presence of Biobeads, was used to 
incorporate the channels into the liposomes. After three to ten hours of incubation with bio-beads (unless explicitly stated), the proteo-liposomes were pelleted down at $100,000 \mathrm{~g}$, resuspended and used for spectroscopic measurements.

\section{V.3.7 EPR Spectroscopy and Data Analysis}

MscS-Cys mutants were purified and spin-labeled using 1:10 molar excess of the label. After reconstitution, their spectra were obtained at room temperature in a Bruker EMX X-band EPR spectrometer. The spectra were compared after normalization with the spin-label number (Perozo et al., 1998). 


\section{V.4 RESULTS AND DISCUSSION}

\section{V.4.1 MscS is a slightly Anion-selective, Pressure Activated Ion Channel}

MscS activity was characterized using an E. coli strain lacking MscS, MscL, and MscK channels (Levina et al., 1999), which ensures that the recorded response corresponds only to that of the recombinant MscS. Patch-clamp experiments were performed on giant-spheroplasts under the inside-out configuration and revealed MscS activity when a gentle pressure (such as $-40 \mathrm{mmHg}$ ) was applied. The current-voltage relation obtained under these conditions is shown in Figure V.2.A and exhibits a slight rectification for positive membrane potentials, which becomes more evident when using asymmetric conditions. The estimated conductance at $200 \mathrm{mM}$ of $\mathrm{KCl}$ is $\sim 1.16 \mathrm{nS}$, in agreement with previous electrophysiological measurements of MscS (Sukharev, 2002; Sukharev et al., 1993). Under asymmetric conditions, the reversal potential is estimated to be $+2.2 \mathrm{mV}$, which is small compared to the theoretical reversal potential for a completely selective anion channel $(+28.4 \mathrm{mV}$ according to the Nernst equation). Using the GHK equation (see Experimental Procedures), the selectivity of the channel is estimated to be $P_{\mathrm{Cl}}{ }^{-} / P_{\mathrm{K}}{ }^{+}=1.19$, slightly smaller than the previously reported ratio of $\sim$ 1.48 (Sukharev, 2002). Thus, MscS seems to be only slightly selective for anions.

Single-channel traces of MscS activity shown in Figure V.2.B exhibit a behavior that depends on the sign of the applied bias. While MscS remains steadily in an open state at hyper-polarizing membrane potentials, the single channel transitions measured for positive biases are less well defined displaying a flickery behavior, likely indicating that under these conditions $\mathrm{MscS}$ adopts multiple and unstable open conformations.

Macroscopic currents and the corresponding nominal open probability of MscS 
(NPo, Figure V.3.A) seem to indicate that the number of open channels depends on the applied voltage. The higher the magnitude of the voltage applied, the lower the open probability (Koprowski and Kubalski, 1998). However, this behavior might be caused by differences in the rate of pressure application as previously suggested (Akitake et al., 2005) and/or differences in the total number of channels available in the corresponding patches. Another reason might be associated with the very slow recovery of $\mathrm{MscS}$ from inactivation (Akitake et al., 2005; Vasquez and Perozo, 2004), coupled with the intrinsic variability of mechanical stimulation of membrane patches (Gil et al., 1999; Zhang et al., 2001). 


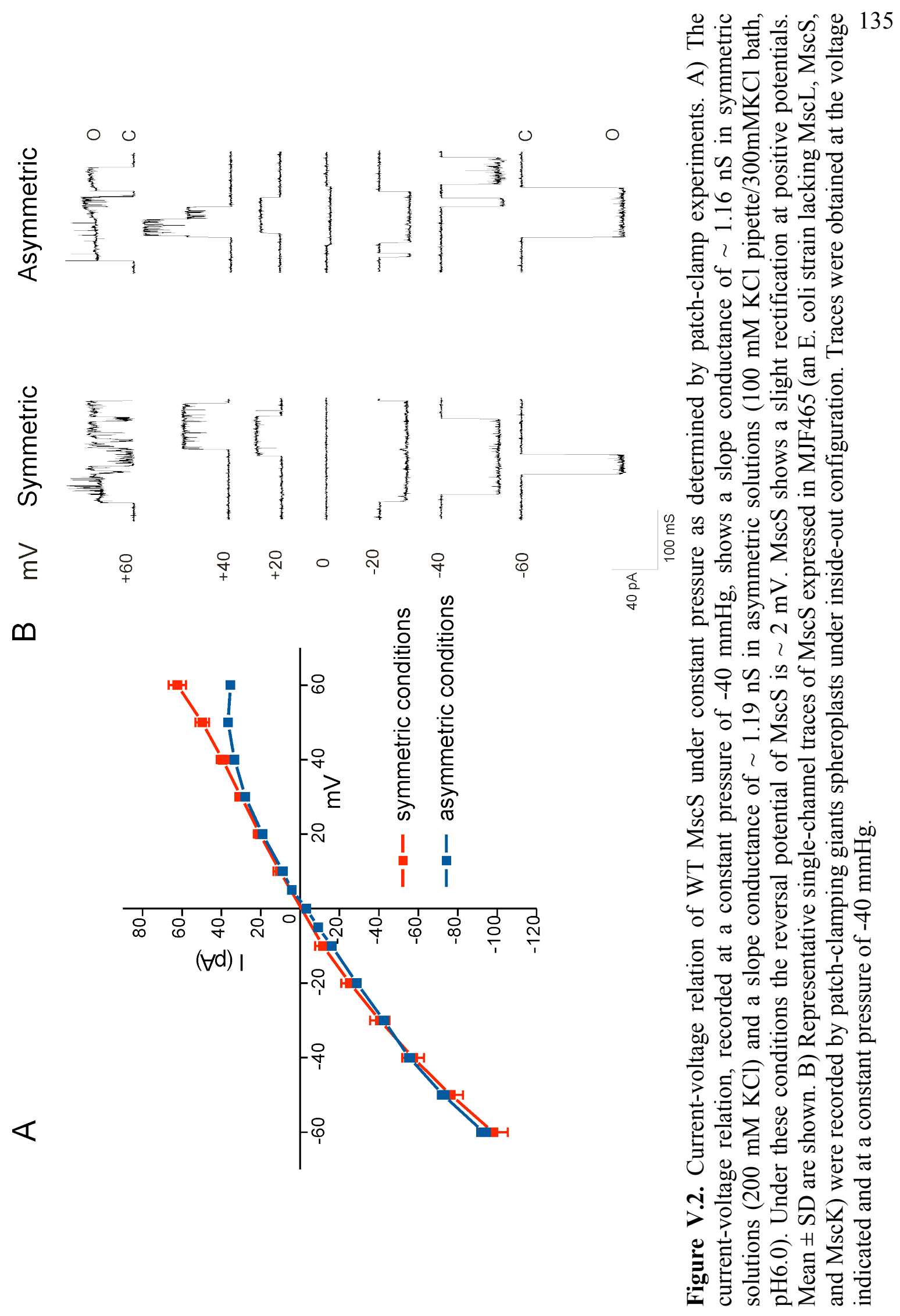


A
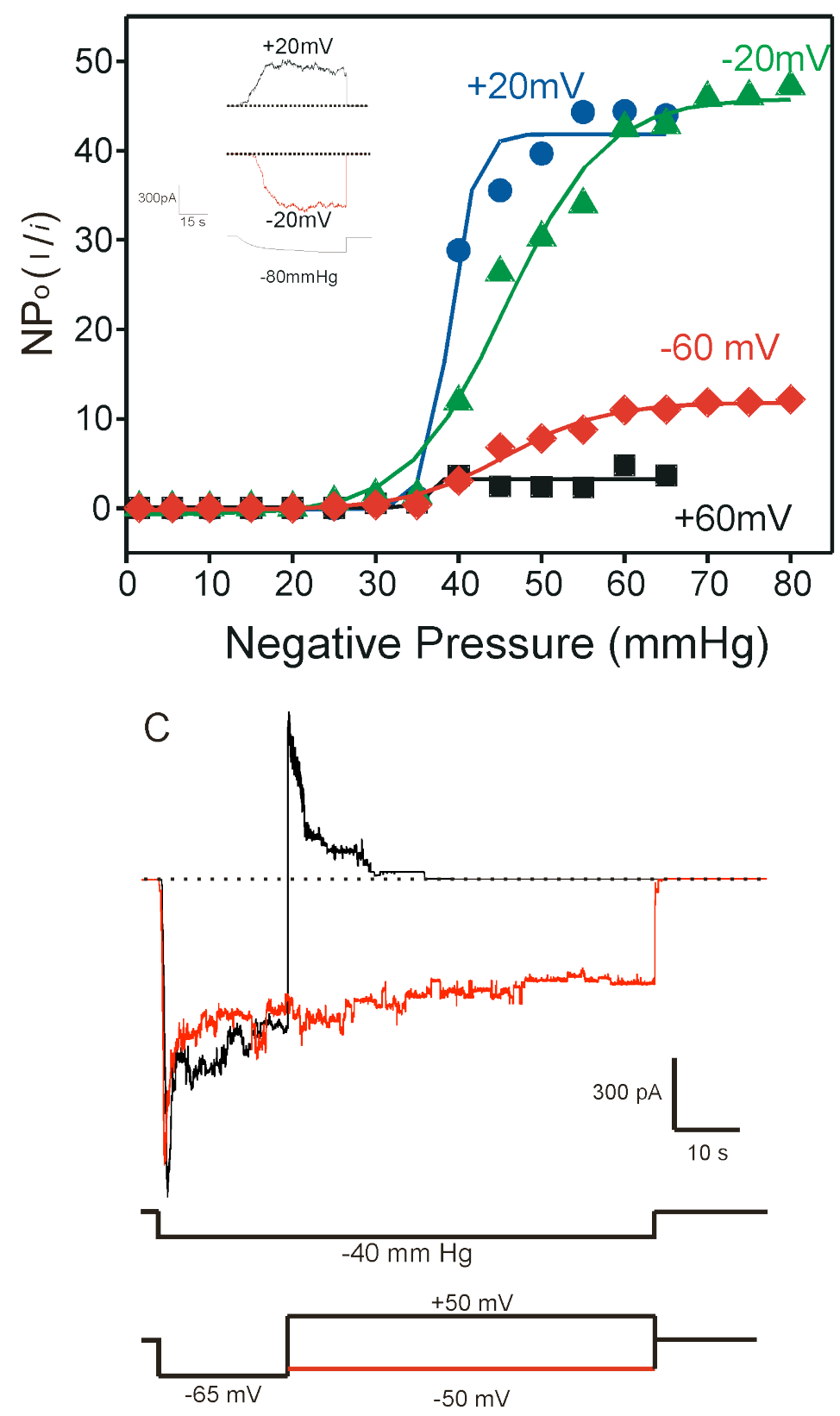

B

136
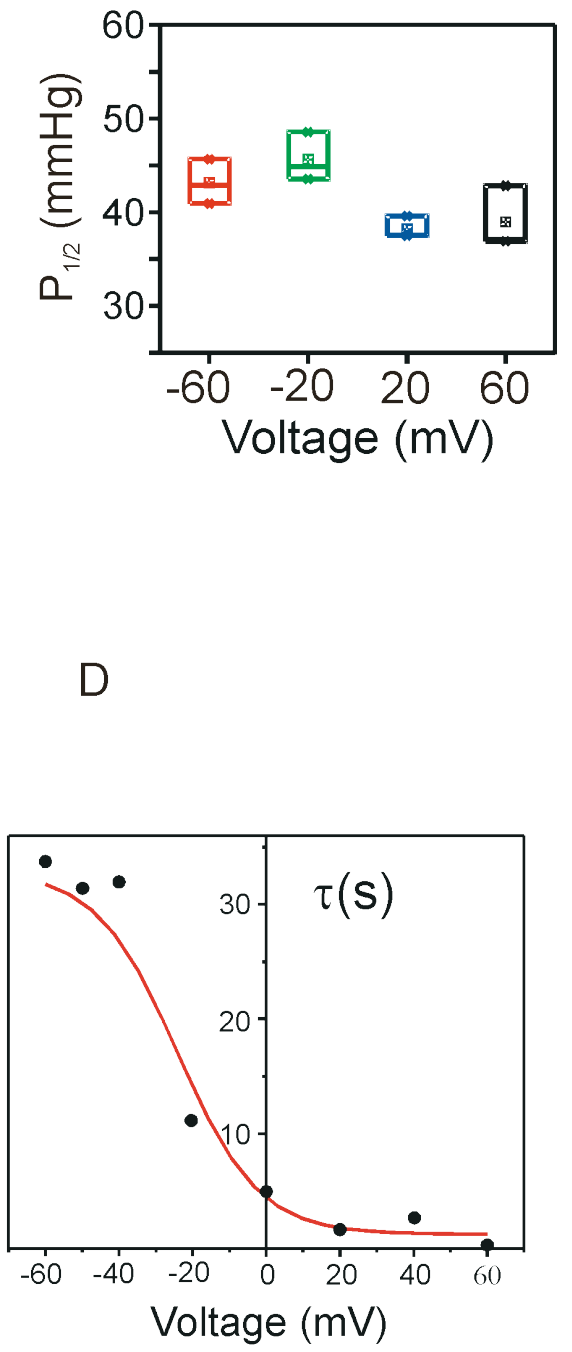

Figure V.3. MscS gating is dependent on tension and modulated by voltage. A) Macroscopic MscS currents adjusted to a Boltzmann-function fit to the NPo (nominal open probability) versus pressure at different transmembrane potentials. The inset shows macroscopic currents (at least 30 channels in the patch) corresponding to $\pm 20 \mathrm{mV}$, elicited by an increase in negative pressure up to $-80 \mathrm{mmHg}$. B) Midpoints of activation $\left(\mathrm{P}_{1 / 2}\right)$ plotted against transmembrane voltage (derived from the Boltzmann-function fit shown in panel A) suggest that opening of $\mathrm{MscS}$ at different potentials has approximately the same $\mathrm{P}_{1 / 2}$ value. Mean \pm SD are shown. C) Macroscopic currents obtained from two-pulse protocol experiments under constant pressure reveal the presence of a voltage-dependent inactivation; the rate and extent of inactivation speeds-up significantly with membrane depolarization. D) Decay time constant $t$ obtained from fitting a single exponential to the macroscopic records obtained from the two-pulse protocol showing the voltage dependence of the inactivation. 
The midpoint of activation determined from NPo is voltage independent, as shown in Figure V.3.B, i.e., the pressure required to open MscS does not depend on the applied voltage. In contrast, a two-pulse voltage protocol used to measure macroscopic currents revealed that $\mathrm{MscS}$ inactivation strongly depends on the applied voltage. Similar to KcsA, a channel from Streptomyces lividans which activation solely depends on proton concentration and its inactivation is modulated by voltage (Chakrapani et al., 2007; Cordero-Morales et al., 2006). Indeed, the time-constant decay of macroscopic currents depends dramatically on the sign of the second voltage pulse (Figure V.3.C and D). The voltage dependence of inactivation appears to be the determinant of channel activity at depolarizing potentials, although the channels also inactivate at negative potentials. The latter result, first reported in Vasquez and Perozo (Vasquez and Perozo, 2004), agrees well with data presented afterward (Akitake et al., 2005; Sotomayor et al., 2007), but differs from previous studies suggesting a voltage-independent adaptation (Koprowski and Kubalski, 1998). The disagreement may be due to differences in the range and/or duration of applied voltages. While voltages utilized in Koprowski and Kubalski (Koprowski and Kubalski, 1998) did not exceed $\pm 30 \mathrm{mV}$, the voltage dependence of inactivation observed here becomes more apparent at voltages larger than $+40 \mathrm{mV}$ (Figure V.3.D).

Electrophysiological experiments presented here and elsewhere indicate that MscS should adopt at least three different conformations (Akitake et al., 2007a; Akitake et al., 2005; Martinac et al., 1987; Sukharev, 2002): an open state that exhibits a conductance of $1 \mathrm{nS}$ and is slightly anionic-selective, a closed state, and an inactive state, which is modulated in a voltage-dependent manner. Multiple intermediate conformations 
of sub-conductance states observed in patch-clamp experiments at positive potentials (see Figure V.2) may exist as well. Based on this unambiguous characterization of MscS, a combination of theoretical and experimental tools to probe the available crystallographic structure of $\mathrm{MscS}$ seems to be a good strategy to determine what functional state this structure represents and to model the remaining conformations of MscS.

\section{V.4.2 MscS Crystal Structure Exhibits Low Conductance and High Anionic}

\section{Selectivity}

In order to determine if $\mathrm{MscS}$ crystal structure was in its open state and how the gating transitions occur, Sotomayor and Schulten (Sotomayor and Schulten, 2004) performed an all-atom molecular dynamics simulation on a system that included the whole crystal structure embedded in a lipid bilayer. After a short equilibration with the protein backbone restrained to the crystal structure, the radius of the TM pore in its narrowest part decreased, indicating a strong tendency of the channel to close in the membrane environment. Moreover, simulations in which gradual elimination of the backbone restraints of the protein resulted in the spontaneous closure (asymmetric) of the TM pore. Whether this occluded state represents a closed, collapsed, or inactive state remains uncertain, but clearly $\mathrm{MscS}$ relaxes to a non-conductive state. In the same study, they also explored $\mathrm{MscS}$ dynamics under external application of pressure. The forces were applied radially pointing away from the center of the channel to the TM helices lining the pore, and the ionic currents were computed in order to determine if this in silico gating really trapped an open conformation. Since, no electrostatic bias was applied only spontaneous diffusion of ions should be observed. However, they observed diffusion 
of charges across the cytoplasmic domain, and not through the TM pore. Moreover, they detected that $\mathrm{Cl}^{-}$ions were accumulated at the edges of the pore, while $\mathrm{K}^{+}$ions seemed to accumulate away from the membrane.

The MscS open conformation (achieved through applied forces) obtained by Sotomayor and Schulten (Sotomayor and Schulten, 2004) was used to carry out multiple short simulations in the presence of large external voltages. This work was done in collaboration with Schulten's Laboratory, therefore we were able to compare MscS conductance under patch-clamp experiments and in silico conditions. Two sets of simulations with MscS backbone atoms restrained to the crystal conformation were performed. A set of three simulations, with its backbone restrained, were performed, in which temperature control was applied to heavy atoms of lipids only (to avoid both artificial heating caused by fields and artificial viscosity) with biasing potentials of 0 , +1.2 , and $-1.2 \mathrm{~V}$ (see Figure V.4.A, B, C, and D). In all cases the TM pore remained in an "open" conformation as observed in the crystal structure, although, side chains but not backbone atoms, were free to move (see Figure V.4.A with the pore featuring a radius of $\sim 3 \AA$ in the constriction zone. 


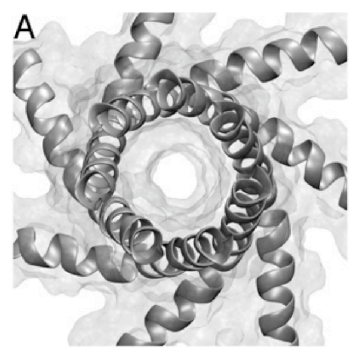

$+1.2 \mathrm{~V}$, restrained

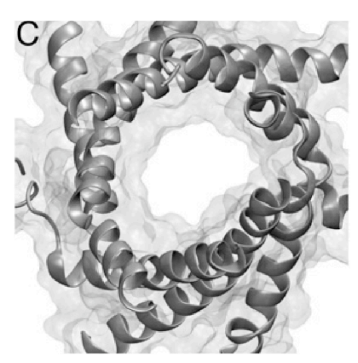

$+1.2 \mathrm{~V}$, unrestrained

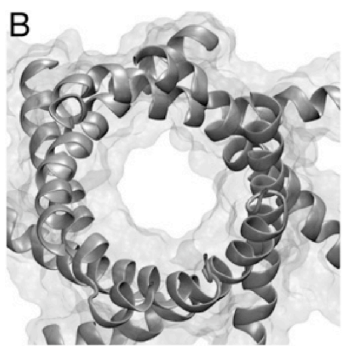

$+1.2 \mathrm{~V}$, unrestrained

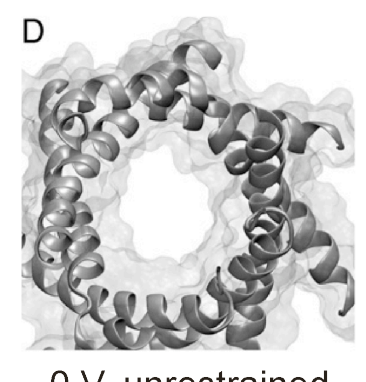

$0 \mathrm{~V}$, unrestrained
$\mathrm{E}$

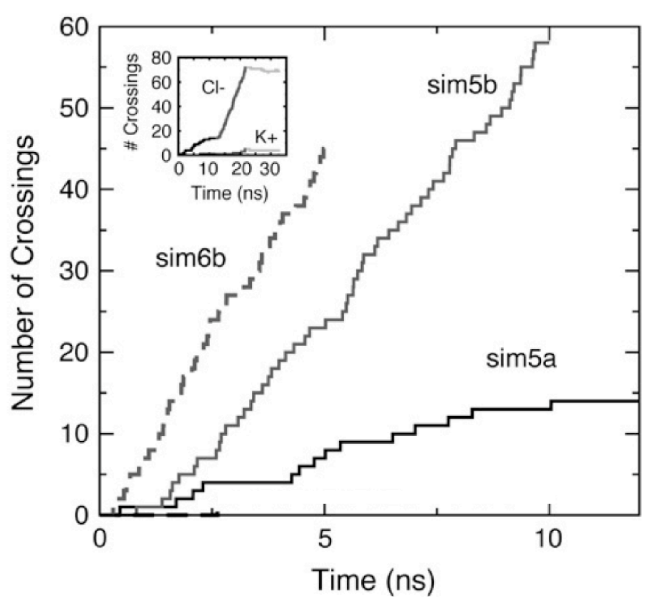

Figure V.4. Ionic currents through MscS as determined by all-atom MD simulations. A-D) Top views of MscS's transmembrane pore (residues 96-128) shown in cartoon and transparent surface representations (residues 96-128) at the end of simulations. A) $\operatorname{sim} 5 \mathrm{a}(+1.2 \mathrm{~V}$, backbone restrained $), \mathrm{B}) \operatorname{sim} 5 \mathrm{~b}(+1.2 \mathrm{~V}$, backbone released; $10 \mathrm{~ns}), \mathrm{C})$ $\operatorname{sim} 6 \mathrm{~b}(+1.2 \mathrm{~V}$, backbone released, $5 \mathrm{~ns})$, and $\mathrm{D}) \operatorname{sim} 5 \mathrm{~d}(0 \mathrm{~V}$, backbone released). E) Ion crossing events $\left(\mathrm{Cl}^{-}\right)$through MscS TM pore versus time for simulations sim5a, $\operatorname{sim} 5 \mathrm{~b}$, and sim6b. A clear steady-state ionic current driven by chloride ions (enhanced upon release of restraints) is observed for simulations $\operatorname{sim} 5 \mathrm{a}, \operatorname{sim} 5 \mathrm{~b}$, and $\operatorname{sim} 6 \mathrm{~b}(+1.2$ V). The inset shows crossing events during $\operatorname{sim} 5 \mathrm{a}, \operatorname{sim} 5 \mathrm{~b}$, and $\operatorname{sim} 5 \mathrm{~d}$. High anionic selectivity is evident. 
Application of external electrostatic potentials resulted, as expected, in transport of ions through the TM pore. Figure V.4.E shows $\mathrm{Cl}^{-}$permeation events throughout simulations in which a $+1.2 \mathrm{~V}$ bias was applied while the protein was restrained to its crystallographic conformation. From the data it is evident that the crystal conformation exhibits low conductance and high selectivity for $\mathrm{Cl}^{-}$. Indeed, meaningful conduction was observed only for simulations at $+1.2 \mathrm{~V}$ and was driven entirely by $\mathrm{Cl}^{-}$ ions. The maximum estimated current for the restrained structure is $\sim 237 \pm 61 \mathrm{pA}$ (Figures V.4A and E), which corresponds to a conductance of $\sim 198 \mathrm{pS}$ at $+1.2 \mathrm{~V}$. If one takes into account the overestimate of bulk $\mathrm{KCl}$ conductivity using identical simulation conditions, the conductance scales down to $\sim 169 \mathrm{pS}$, a value that is small compared to the expected $1 \mathrm{nS}$ conductance of the fully-open channel under experimental conditions. Furthermore, reversal of the applied potential to -1.2 and $-0.6 \mathrm{~V}$ resulted in negligible ion transport.

The small currents and strong rectification observed from MD simulations for negative biasing potentials contrast with the experimental data showing only a weak rectification that becomes more apparent under asymmetrical conditions (Figure V.2.A). The discrepancy may be a consequence of the large voltages used in the simulations, since the maximum current flowing through the channel is limited by free diffusion of ions being supplied to the periplasmic and cytoplasmic ends of the pore (Hille, 2001). However, the same behavior was observed in coarse-grained simulations of the $\mathrm{MscS}$ crystal conformation lasting $100 \mathrm{~ns}$ each and using smaller biases of $\pm 50 \mathrm{mV}$ and \pm 100 $\mathrm{mV}$ (Sotomayor et al., 2006). Moreover, crude estimates of MscS conductance presented in Sotomayor and Schulten (Sotomayor and Schulten, 2004) and Anishkin and Sukharev 
(Anishkin and Sukharev, 2004) agree well with the observed behavior. Overall, different computational results (obtained in some cases from independent groups) seem to converge to the same conclusion.

All-atom MD simulations of $\mathrm{MscS}$ described above, along with previous work focusing on gating and stability of MscS (Sotomayor and Schulten, 2004) as well as MscS electrostatic properties (Sotomayor et al., 2006), reveal dynamical and permeation properties of the entire crystal structure of $\mathrm{MscS}$ in its native-like environment under time-scales from tens to hundreds of nanoseconds. Such realistic simulations enable measurements of ionic currents that can be readily compared to experimental ionic currents determined through patch-clamp experiments. The simulations suggest that the conformation depicted by the crystal structure of MscS (Bass et al., 2002) represents an intermediate or inactive state, since its conductance is small and it is highly selective for anions. On the other hand, closed states, obtained upon release of restraints applied to the backbone of the structure in the absence of tension or external biases, are evidently nonconducting.

\section{V.4.3 Locating the Charges Responsible for the Voltage Dependence of the}

\section{Inactivation in MscS}

An attractive mechanism to explain the concerted effects of tension and voltage on $\mathrm{MscS}$ has been based on the possible conformational changes of the TM1-TM2 helical hairpin and the location of the charges, which are apparently exposed to lipids in the crystal structure (see Figure V.5.A). In this model, the resting state is characterized by a compact conformation with all TM helices tightly packed around the pore; the open state would be favored by membrane hyper-polarization since a concerted outward 
movement of helices will result in wetting and expansion of the pore constriction. On the other hand, depolarizing potentials would push the helices toward the extracellular side and thus favoring their detachment from the core TM3 helices and favor the transition to sub-conducting states followed by the inactivated state (Akitake et al., 2005).

To explore this hypothesis, we used a high throughput assay that depends on the ability of MscS to protect $E$. coli when it is subjected to abrupt osmotic changes. The TM charged residues were neutralized by glutamine substitutions (Figure V.5.B). Figure V.5.B shows that none of the neutralized charges in the TM segments generated LOF phenotypes. However, R59Q and K60Q show an intermediate response. From the crystal structure, $\operatorname{Arg} 46$ was considered the most likely candidate for the voltage sensing process of MscS, since its side-chain appeared to be not only exposed but in the middle of the lipid bilayer. For this reason, even though we did not find any difference between the WT and R46Q response, we decided to further investigate this mutant. We characterized the voltage dependence of R46Q (Figure V.5.C), and we found that the time constant of inactivation is within the noise of the WT. When we evaluated the location of the spinlabeled R46C on MscS closed conformation in the context of a membrane (see chapter III and Vasquez et al 2008), we found that this position was not exposed to the non-polar region of the membrane, instead was buried within the protein despite the fact that the spin-label group is less polar than the Arginine side-chain. Another group (Takeshi et al., 2006b) confirmed our results by patch-clamp experiments, since they could not find any change in the voltage modulation when they mutated to either positive or negative charges along the TM segments. Same group also found that mutations at position Arg59 
and Lys60 made MscS more difficult to open, but there was no relation with MscS

voltage dependence (Nomura et al., 2007).

A

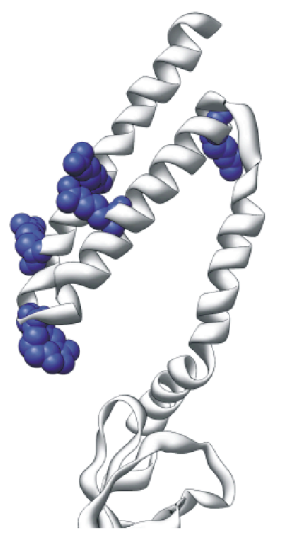

B
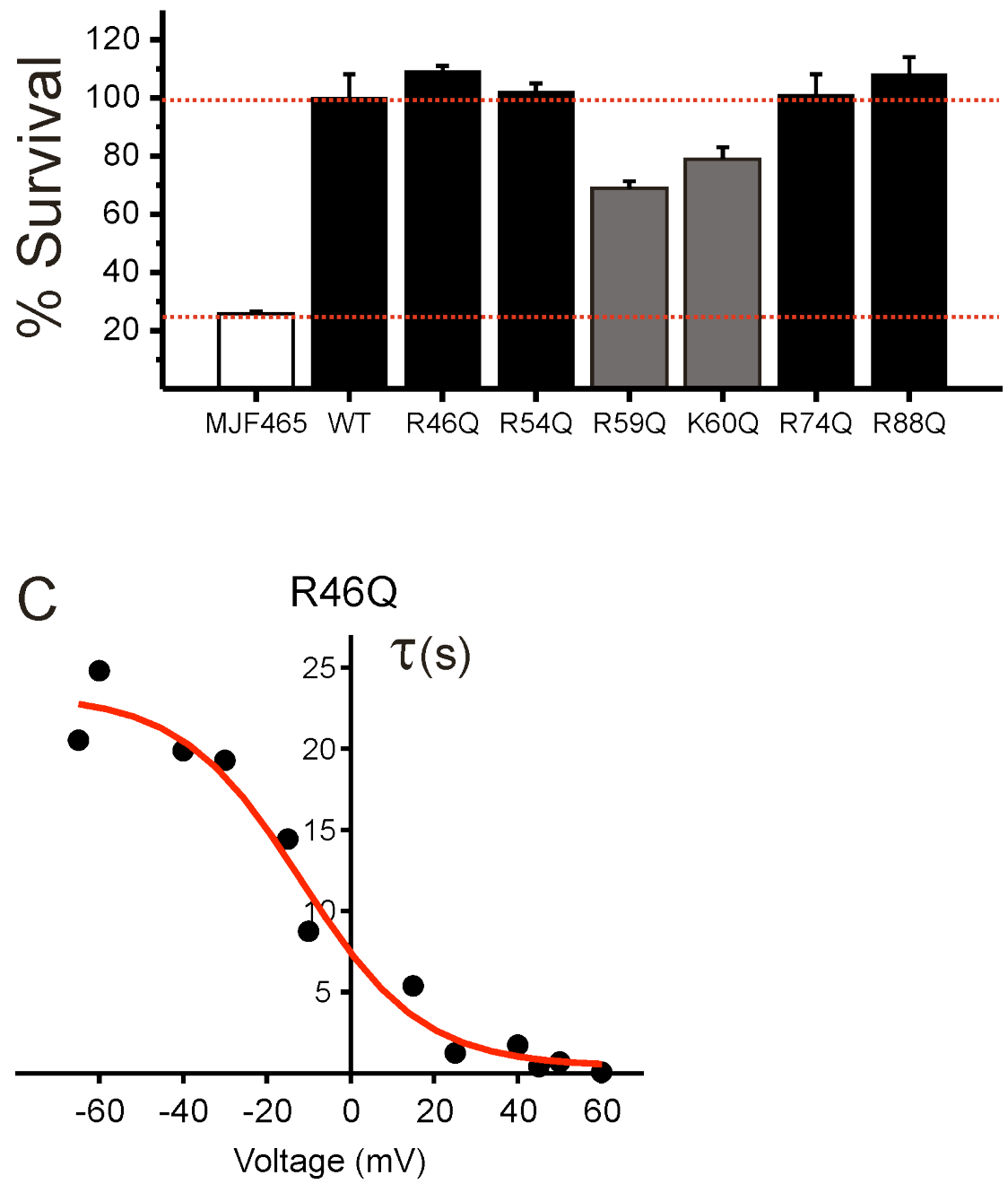

Figure V.5. Neutralization of charged residues in the TM1-TM2 hairpin. A) Ribbon diagram showing in blue space-filling representations the residues that were neutralized by substitution with a glutamine. B) Functional assay of cells containing glutamine mutants over-expressed in pQE32 vector. MJF465 and WT MscS were used as a negative and positive control, respectively. Red dotted lines represent the mean value of WT MscS (top) and the MJF465 (bottom). C) Voltage dependence of the inactivation-time constant $\tau$. Inactivation decay time constants were fitted to a single exponential function. 
Since the TM charges did not show any evidence of being involved in MscS voltage-dependence, we decided to further investigate other options. Based on a earlier paper (Sotomayor and Schulten, 2004) which revealed that during the asymmetric closure of $\mathrm{MscS}$, when equilibrated in a relaxed membrane, a salt bridge formation occurred between conserved residues (Asp62 and Arg128) of adjacent subunits (Figure V.6.A). Motivated by this observation of novel interactions between specific residues during MD trajectories, we carried out multiple functional assays on selected mutants of MscS. Since residues Asp62 and Arg128 were observed to form salt-bridges throughout simulations, mutations were performed at positions Asp2, Arg128, and Arg131, which are in close proximity. These residues are located at the loop connecting TM1 and TM2 helices and at the end of TM3B.

We report here multiple LOF phenotypes for MscS mutants in which the charged nature of wild-type residues located at positions 62, 128, and 131 are modified (Figure V.6). Neutralization of the charge at position Asp62 generates the same phenotype as if the cell did not have any mechanosensitive channel. It seems that the presence of a negative charge at this position is required for $\mathrm{MscS}$ to protect the cell. Moreover, when a Glutamate (Nomura et al., 2007) is substituted at this position, MscS displays a WT behavior. On the other hand, mutations at positions Arg128 and Arg131 did not cause such an extreme response, as we found a 40 or $50 \%$ survival. The construction of negative (D62/R128D/R131D) or positive (D62R/R128/R131) triads also generated extreme LOF phenotypes (see Figure V.6 bottom). These results indicated that apart from interaction, the charge balance is also important in this inter-connective region (compare with the triple mutant D62N/R128Q/R131Q result). We were able to partially recapitulate 
the salt bridge interaction with the double mutants D62R/R128D and D62R/R131D (see red stars in bottom Figure V.6). However, D62R/R131D seems a more appropriate candidate for the salt bridge because it shows an $85 \%$ of cell recovery. This partial recovery of the interaction may depend on the geometrical orientation of the charges, which is a possible reason for the low survival of D62R/R128D pair.

Given the fact that we found several LOF mutants, we decided to check their incorporation in the E. coli membrane, as well as their oligomeric stability. For this purpose, we expressed and purified WT MscS and all of the charged mutants (sixteen of them). We were able to conclude that there were no differences, regarding expression, membrane incorporation, and oligomeric stability (Figure V.7.A and B). Hence, the response to the osmotic downshock is only coming from the ability of the channel to properly gate upon changes in lateral membrane tension.

In addition, we purified $\mathrm{D} 62 \mathrm{C} / \mathrm{R} 128 \mathrm{C}$, and $\mathrm{D} 62 \mathrm{C} / \mathrm{R} 131 \mathrm{C}$ (with their respective controls D62C, R128C, and R131C), spin-labeled and reconstituted in liposomes in order to detect proximity by EPR spectroscopy. For both double-mutants we did not obtain any spin-spin coupling (see Figure V.7.C). This result indicates that the formation of the saltbridges might occur in the open or inactive state, but not in the closed conformation. The non-polar location of spin labeled D62C, according to our closed state EPR model (see chapter II and Vasquez et al 2008), helped us understand why Asp62 is not in position to form an electrostatic interaction with Arg128 or Arg131. This outcome was also confirmed by cross-linking experiments (Nomura et al., 2007), since the formation of disulfide bonds only occurred when the cells, carrying the mutants, were subjected to hypo-osmotic dilution. The influence of Asp62, Arg128, and Arg131 in the voltage 
dependence process of $\mathrm{MscS}$ is not yet clear, as Nomura and collaborators (Nomura

et al., 2007) demonstrated that the voltage dependence of the inactivation was still present on charge neutralization, even though D62N has a faster rate of inactivation than WT MscS.

All of the snapshots, obtained by MD simulations, showed salt bridge formation between residue Asp62 of one subunit and Arg128 of the subsequent subunit. These two residues and Arg131 are conserved in at least $65 \%$ of the sequences of 25 homologs of MscS in prokaryotes and archaea (see Figure V.1). Moreover, when the residues are not identical in those three positions they are being replaced by a Glutamate residue in position 62, also negatively charged, and by a Lysine residue in position 128 and 131, also positively charged. These observations indicate a strong conservation of charge that will favor the formation of the respective salt bridge in similar structures. However, the specific role of these salt bridges in the gating mechanism is not clear, but they might provide a direct link between the movement of the TM1-TM2 domains and the pore lining helices. 

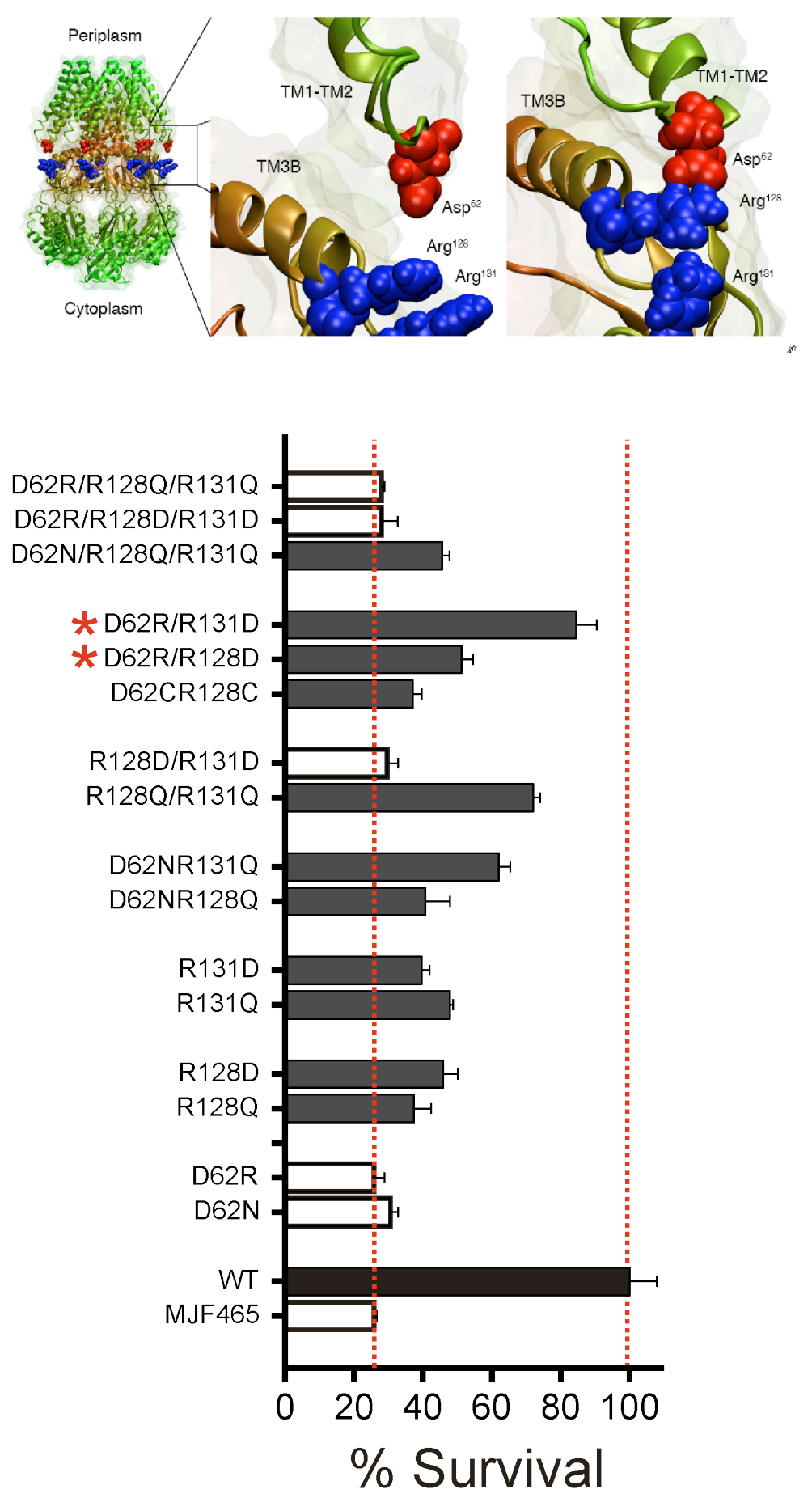

Figure V.6. Salt-bridge formation as determined through all-atom MD simulations of $\mathrm{MscS}$ and Functional Assays. Top) Crystal structure of $\mathrm{MscS}$ shown in cartoon and transparent surface representations. Side chains of residues Asp62 (red), Arg128 (blue), and Arg131 (blue) are shown in space-filling representation. Zoom in) Detail of MscS structure showing the TM1-TM2 loop of one subunit and the TM3B helix of the adjacent subunit. Snapshots correspond to the crystal structure (left) and a conformation obtained after $15 \mathrm{~ns}$ of molecular dynamics simulations (right). Bottom) Functional assay of cells containing mutations of residues putatively involved in salt bridge formation. MJF465 and WT MscS were used as a negative and positive control, respectively. Red dotted lines represent the mean value of WT MscS (right) and the mean value of MJF465 (left). 
A

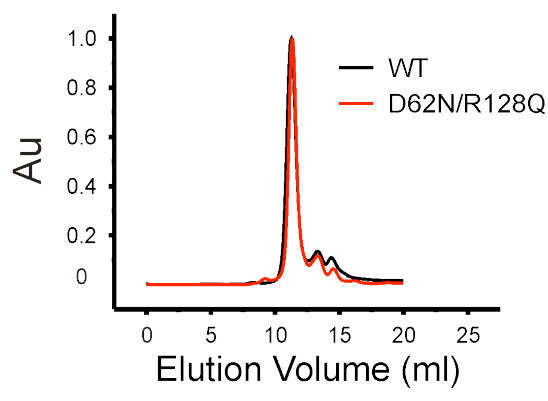

C

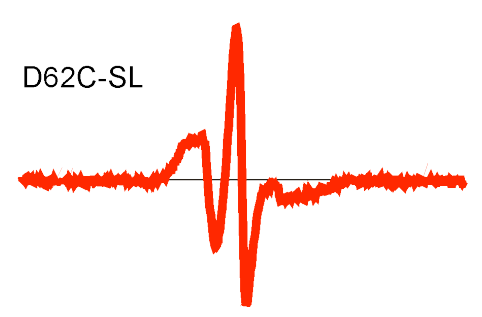

B
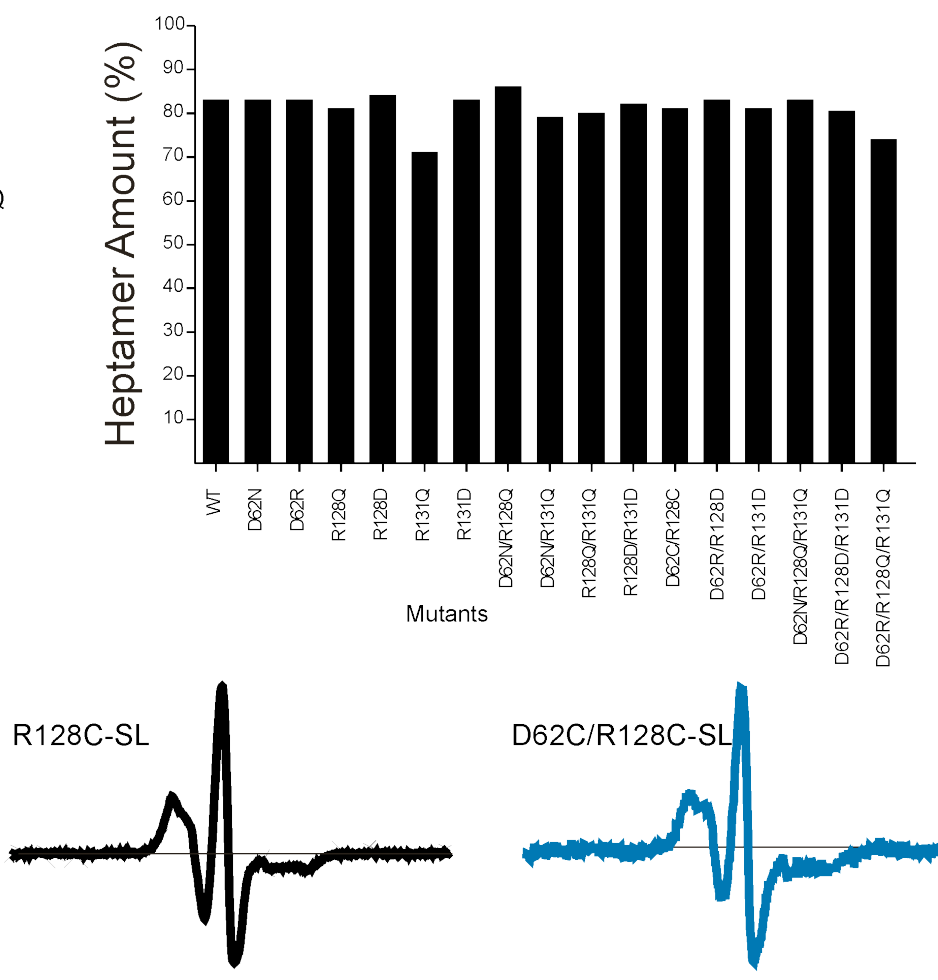

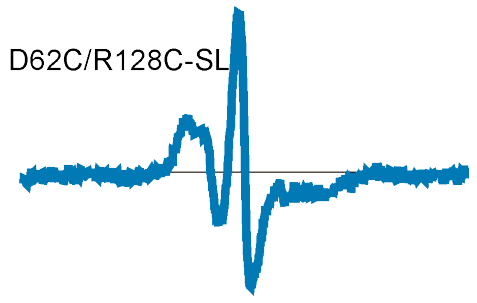

Figure V.7. Hydrodynamic properties of MscS and EPR spectroscopy of MscS salt bridge mutants. A) Hydrodynamic properties of WT MscS and MscS double-mutant D62N/R128Q evaluated from gel filtration elution volume. B) Yield of purified oligomeric WT MscS and putative salt bridge mutants obtained from the expression in MJF453 E. coli cells, evaluated from the elution volume in a gel filtration column. C) CW-EPR spectra obtained from spin labels attached at MscS residues D62C, R128C, and $\mathrm{D} 62 \mathrm{C} / \mathrm{R} 128 \mathrm{C}$, after reconstitution in DOPC:POPG. 


\section{CHAPTER VI: Conclusions and Future Perspectives}

Mechanosensitive channels are considered to function as mechano-electrical switches in diverse physiological processes such as touch, hearing, turgor control and osmoregulation. These types of channels are able to transduce lipid deformation forces in the bilayer into a sequence of structural rearrangements that lead to changes in membrane permeability (as the transbilayer pressure increases the channel probability also increases). Therefore, mechanically gated ion channels are the link between physical forces and biological responses.

The present thesis was aimed to understand the molecular basis for mechanosensation, in order to get a glimpse of the process that involves force translation into protein motion. For this we have used MscS a mechanosensitive channel from $E$. coli, which structure has been already solved, and its family is widespread in at least five life kingdoms (Plantae, Fungi, Protoctista, Monera, and Archaea). It is worth mentioning that prokaryotic mechanosensitive channels are structurally and mechanistically unrelated to those found in animals in which association with cytoskeletal proteins is believed to play a critical role in the transduction of mechanical deformations.

$\mathrm{MscS}$ is activated by changes in membrane tension and its inactivation is modulated by TM voltage, its crystal structure displayed a homoheptamer, in which each subunit has three TM segments and a large cytoplasmic domain. During the course of this research we found out that the available protocols for purifying and reconstituting MscS were not suitable for spectroscopical approaches. For this reason we had to dedicate a significant amount of time to solve this inconvenient. We were able to establish a highquality biochemical preparation to routinely express and purify MscS. Later on, these 
tactics helped us in the elucidation of the molecular mechanism of tension dependent gating.

We made use of these biochemical approaches to analyze the structural dynamic properties of membrane-reconstituted $\mathrm{MscS}$ in the closed state conformation, as determined by site-directed spin labeling, EPR spectroscopy and computational modeling. We have used the experimental data to refine the crystal structure under known physiological conditions, and to construct a model of the closed conformation of MscS. The new closed model showed significant differences regarding the position of the TM1TM2 hairpin, and the arrangement of TM2 with respect to TM3. We find that the previously unresolved $\mathrm{NH}_{2}$-terminus is a mixed helical hairpin, placed at the periplasmic interface of the lipid bilayer, away from the channel central axis of symmetry. Our experimental data suggested that the $\mathrm{MscS}$ closed state is in a different and a more compact conformation than the one trapped in the crystal structure.

We were also able to determine that a cone-shaped amphiphile (lyso PC) activates $\mathrm{MscS}$ and its incorporation into the membrane does not allow its inactivation. Since we stabilized the open conformation of MscS by modifying the TM tension profile through the incorporation of lyso $\mathrm{PC}$, the structural rearrangements upon gating of $\mathrm{MscS}$ were monitor. We collected mobility and accessibility data of 128 spin-labeled mutants that span the TM domain. With this pattern of global accessibilities and mobilities obtained by CW-EPR spectroscopy we are currently building a three-dimensional model of $\mathrm{MscS}$ in an open conformation in order to elucidate the conformational changes during gating. Nonetheless the analysis of individual mutants allow us to propose that upon opening the $\mathrm{NH}_{2}$ terminus of $\mathrm{MscS}$ penetrates into the membrane, the TM1-TM2 hairpin tilts and 
moves away from the TM3 segment, the pore increases its diameter, the TM3 helices rotate, and wetting of the permeation pathway occurs. MscS at least could adopt four different conformational states: closed, open, inactivated, and desensitized. With this collection of spectroscopical data we have been able to elucidate two relevant states (closed and open) in the gating pathway of $\mathrm{MscS}$, providing a physical framework to understand the activation of MscS.

Once the three-dimensional model of $\mathrm{MscS}$ in an open conformation is ready, we have to test its stability using molecular dynamics simulations, as we previously did with the closed conformation. We will also measure water penetration using approaches such as HOLE profile, to qualitatively determine the radius pore in the trapped open conformation. Prior publication, we are planning to further characterize the effect of lyso PC in both leaflets of the membrane, as well as the response of some cysteine-labeled mutants in the pore helix, to determine their maximal response under lyso PC adsorption

In a different but connected topic, we related electrophysiological measurements and conformation of the crystal. We characterized the response of MscS to voltage and pressure by patch-clamp technique, and further evaluated by molecular dynamics simulations. Electrophysiological experiments revealed that $\mathrm{MscS}$ is a slightly anionselective channel with a conductance of $\sim 1 \mathrm{~ns}$, activated by pressure and inactivated in a voltage-dependent manner. In addition, simulations showed that MscS crystal conformation exhibits low conductance, mainly from $\mathrm{Cl}^{-}$ions. Since the influence of voltage on MscS inactivation remains unknown, we studied the effect of several charged residues that according to the crystal structure were facing the lipids, and of some other residues that were putatively interacting through salt bridges as determined by molecular 
dynamics simulations. Neutralization of TM charged residues did not affect MscS ability to protect against sudden osmotic changes, and did not eliminate the voltage dependence of inactivation. Moreover, the disruption of the salt bridge interaction did not affect the voltage dependence, but interestingly generated loss of function phenotypes. More studies are needed in order to understand the voltage-dependence of the inactivation process in $\mathrm{MscS}$. 


\section{REFERENCES}

Akitake, B., Anishkin, A., Liu, N., and Sukharev, S. (2007a). Straightening and sequential buckling of the pore-lining helices define the gating cycle of MscS. Nat Struct Mol Biol 14, 1141-1149.

Akitake, B., Anishkin, A., and Sukharev, S. (2005). The "dashpot" mechanism of stretchdependent gating in MscS. J Gen Physiol 125, 143-154.

Akitake, B., Spelbrink, R. E., Anishkin, A., Killian, J. A., de Kruijff, B., and Sukharev, S. (2007b). 2,2,2-Trifluoroethanol Changes the Transition Kinetics and Subunit Interactions in the Small Bacterial Mechanosensitive Channel MscS. Biophys J 92, 2771-2784.

Altenbach, C., Marti, T., Khorana, H. G., and Hubbell, W. L. (1990). Transmembrane protein structure: spin labeling of bacteriorhodopsin mutants. Science 248, 1088-1092. Anishkin, A., Akitake, B., and Sukharev, S. (2008). Characterization of the resting MscS: modeling and analysis of the closed bacterial mechanosensitive channel of small conductance. Biophys J 94, 1252-1266.

Anishkin, A., and Sukharev, S. (2004). Water dynamics and dewetting transitions in the small mechanosensitive channel MscS. Biophys J 86, 2883-2895.

Bass, R. B., Strop, P., Barclay, M., and Rees, D. C. (2002). Crystal structure of Escherichia coli MscS, a voltage-modulated and mechanosensitive channel. Science 298, $1582-1587$.

Berrier, C., Besnard, M., Ajouz, B., Coulombe, A., and Ghazi, A. (1996). Multiple mechanosensitive ion channels from Escherichia coli, activated at different thresholds of applied pressure. J Membr Biol 151, 175-187. 
Bezanilla, F., and Perozo, E. (2002). Structural biology. Force and voltage sensors in one structure. Science 298, 1562-1563.

Brooks, B., Bruccoleri, R. E., Olafson, B. D., States, D. J., Swaminathan, S., and Karplus, M. (1983). CHARMM: A Program for Macromolecular Energy, Minimization, and Dynamics Calculations. J Comp Chem 4, 187-217.

Buechner, M., Delcour, A. H., Martinac, B., Adler, J., and Kung, C. (1990). Ion channel activities in the Escherichia coli outer membrane. Biochim Biophys Acta 1024, 111-121. Chakrapani, S., Cordero-Morales, J. F., and Perozo, E. (2007). A quantitative description of KcsA gating I: macroscopic currents. J Gen Physiol 130, 465-478.

Chang, G., Spencer, R. H., Lee, A. T., Barclay, M. T., and Rees, D. C. (1998). Structure of the MscL homolog from Mycobacterium tuberculosis: a gated mechanosensitive ion channel. Science 282, 2220-2226.

Cordero-Morales, J. F., Cuello, L. G., and Perozo, E. (2006). Voltage-dependent gating at the KcsA selectivity filter. Nat Struct Mol Biol 13, 319-322.

Corey, D. P. (2003). New TRP channels in hearing and mechanosensation. Neuron 39, 585-588.

Cornette, J. L., Cease, K. B., Margalit, H., Spouge, J. L., Berzofsky, J. A., and DeLisi, C. (1987). Hydrophobicity scales and computational techniques for detecting amphipathic structures in proteins. J Mol Biol 195, 659-685.

Cortes, D. M., and Perozo, E. (1997). Structural dynamics of the Streptomyces lividans $\mathrm{K}+$ channel (SKC1): oligomeric stoichiometry and stability. Biochemistry 36, 1034310352. 
Cuello, L. G., Cortes, D. M., and Perozo, E. (2004). Molecular architecture of the KvAP voltage-dependent K+ channel in a lipid bilayer. Science 306, 491-495.

Delcour, A. H., Martinac, B., Adler, J., and Kung, C. (1989). Modified reconstitution method used in patch-clamp studies of Escherichia coli ion channels. Biophys J 56, 631636.

Eaton, S. S., Eaton, G. R., and Berliner, L. J. (2005). Biomedical EPR, Part B: Methodology, Instrumentation, and Dynamics., Vol 24: Kluwer Academic/Plenum Publishers).

Edwards, M. D., Li, Y., Kim, S., Miller, S., Bartlett, W., Black, S., Dennison, S., Iscla, I., Blount, P., Bowie, J. U., and Booth, I. R. (2005). Pivotal role of the glycine-rich TM3 helix in gating the MscS mechanosensitive channel. Nat Struct Mol Biol 12, 113-119. Fanucci, G. E., and Cafiso, D. S. (2006). Recent advances and applications of sitedirected spin labeling. Curr Opin Struct Biol 16, 644-653.

Farahbakhsh, Z. T., Altenbach, C., and Hubbell, W. L. (1992). Spin labeled cysteines as sensors for protein-lipid interaction and conformation in rhodopsin. Photochem Photobiol $56,1019-1033$.

Garcia-Anoveros, J., and Corey, D. P. (1996). Touch at the molecular level. Mechanosensation. Curr Biol 6, 541-543.

Gross, A., and Hubbell, W. L. (2002). Identification of protein side chains near the membrane-aqueous interface: a site-directed spin labeling study of KcsA. Biochemistry $41,1123-1128$.

Gullingsrud, J., Kosztin, D., and Schulten, K. (2001). Structural determinants of MscL gating studied by molecular dynamics simulations. Biophys J 80, 2074-2081. 
Hackney, C. M., and Furness, D. N. (1995). Mechanotransduction in vertebrate hair cells: structure and function of the stereociliary bundle. Am J Physiol 268, C1-13.

Hamill, O. P., and Martinac, B. (2001). Molecular basis of mechanotransduction in living cells. Physiol Rev 81, 685-740.

Hille, B. (2001). Ionic Channels of Excitable Membranes, 3rd edn (Sunderland, MA:

Sinauer Associates).

Hubbell, W. L., Gross, A., Langen, R., and Lietzow, M. A. (1998). Recent advances in site-directed spin labeling of proteins. Curr Opin Struct Biol 8, 649-656.

Iscla, I., Levin, G., Wray, R., and Blount, P. (2007). Disulfide Trapping the Mechanosensitive Channel MscL into a Gating-Transition State. Biophys J 92, 12241232.

Jasti, J., Furukawa, H., Gonzales, E. B., and Gouaux, E. (2007). Structure of acid-sensing ion channel 1 at 1.9 A resolution and low pH. Nature 449, 316-323.

Jiang, Y., Lee, A., Chen, J., Ruta, V., Cadene, M., Chait, B. T., and MacKinnon, R. (2003). X-ray structure of a voltage-dependent K+ channel. Nature 423, 33-41.

Jorgensen, W. L., Chandrasekhar, J. D., Madura, J. D., Impey, R. W., and Klein, M. L. (1983). Comparison of Simple Potential Functions for Simulating Liquid Water. Journal of Chemical Physics 79, 926-935.

Kabsch, W., and Sander, C. (1983). Dictionary of protein secondary structure: pattern recognition of hydrogen-bonded and geometrical features. Biopolymers 22, 2577-2637. Kellenberger, S., and Schild, L. (2002). Epithelial sodium channel/degenerin family of ion channels: a variety of functions for a shared structure. Physiol Rev 82, 735-767. 
Kloda, A., and Martinac, B. (2002). Common evolutionary origins of mechanosensitive ion channels in Archaea, Bacteria and cell-walled Eukarya. Archaea 1, 35-44.

Kocer-Sagiroglu, A., Bulten, E., Walko, M., Feringa, B., Robillard, G., and Meijberg, W. (2005). A sensory valve in liposomal drug delivery systems. J Control Release 101, 374376.

Kocer, A., Walko, M., Meijberg, W., and Feringa, B. L. (2005). A light-actuated nanovalve derived from a channel protein. Science 309, 755-758.

Koprowski, P., and Kubalski, A. (1998). Voltage-independent adaptation of mechanosensitive channels in Escherichia coli protoplasts. J Membr Biol 164, 253-262. Kuo, A., Gulbis, J. M., Antcliff, J. F., Rahman, T., Lowe, E. D., Zimmer, J., Cuthbertson, J., Ashcroft, F. M., Ezaki, T., and Doyle, D. A. (2003). Crystal structure of the potassium channel KirBac1.1 in the closed state. Science 300, 1922-1926.

Kuo, M. M., Saimi, Y., Kung, C., and Choe, S. (2007). Patch clamp and phenotypic analyses of a prokaryotic cyclic nucleotide-gated $\mathrm{K}+$ channel using Escherichia coli as a host. J Biol Chem 282, 24294-24301.

Lazaridis, T., and Karplus, M. (1999). Effective energy function for proteins in solution. Proteins 35, 133-152.

Lemmon, M. A., Flanagan, J. M., Hunt, J. F., Adair, B. D., Bormann, B. J., Dempsey, C. E., and Engelman, D. M. (1992). Glycophorin A dimerization is driven by specific interactions between transmembrane alpha-helices. J Biol Chem 267, 7683-7689. Levina, N., Totemeyer, S., Stokes, N. R., Louis, P., Jones, M. A., and Booth, I. R. (1999). Protection of Escherichia coli cells against extreme turgor by activation of $\mathrm{MscS}$ and 
MscL mechanosensitive channels: identification of genes required for MscS activity. Embo J 18, 1730-1737.

Li, Y., Moe, P. C., Chandrasekaran, S., Booth, I. R., and Blount, P. (2002). Ionic regulation of MscK, a mechanosensitive channel from Escherichia coli. Embo J 21, 5323-5330.

Lundbaek, J. A., and Andersen, O. S. (1994). Lysophospholipids modulate channel function by altering the mechanical properties of lipid bilayers. J Gen Physiol 104, 645673.

Machiyama, H., Tatsumi, H., and Sokabe, M. (2008). Cytoplasmic domain of the bacterial mechanosenstive channel MscS swells up associated with channel-opening. Biophysical Society Meeting, 123A.

MacKerell, A. D., Bashford, D., Bellot, M., Dunbrack, R. L., Evanseck, J. D., Field, M. J., Fisher, S., Gao, J., Guo, H., Ha, S., et al. (1998). All-Atom Empirical Potential for Molecular Modeling and Dynamics Studies of Proteins. Journal of Physical Chemistry B $102,3586-3616$.

Mackerell, A. D., Jr., Feig, M., and Brooks, C. L., 3rd (2004). Extending the treatment of backbone energetics in protein force fields: limitations of gas-phase quantum mechanics in reproducing protein conformational distributions in molecular dynamics simulations. $\mathbf{J}$ Comput Chem 25, 1400-1415.

Maingret, F., Patel, A. J., Lesage, F., Lazdunski, M., and Honore, E. (2000). Lysophospholipids open the two-pore domain mechano-gated $\mathrm{K}(+)$ channels TREK-1 and TRAAK. J Biol Chem 275, 10128-10133. 
Markin, V. S., and Sachs, F. (2007). Thermodynamics of Mechanosensitivity, Vol 58: Elsevier Inc).

Martinac, B. (2001). Mechanosensitive channels in prokaryotes. Cell Physiol Biochem $11,61-76$

Martinac, B. (2004). Mechanosensitive ion channels: molecules of mechanotransduction. J Cell Sci 117, 2449-2460.

Martinac, B., Adler, J., and Kung, C. (1990). Mechanosensitive ion channels of E. coli activated by amphipaths. Nature 348, 261-263.

Martinac, B., Buechner, M., Delcour, A. H., Adler, J., and Kung, C. (1987). Pressuresensitive ion channel in Escherichia coli. Proc Natl Acad Sci U S A 84, 2297-2301. McHaourab, H. S., Lietzow, M. A., Hideg, K., and Hubbell, W. L. (1996). Motion of spin-labeled side chains in T4 lysozyme. Correlation with protein structure and dynamics. Biochemistry 35, 7692-7704.

McHaourab, H. S., and Perozo, E. (2002). Determination of protein folds and conformational dynamics using spin-labeling EPR spectroscopy, Vol 19: Springer). Miller, S., Bartlett, W., Chandrasekaran, S., Simpson, S., Edwards, M., and Booth, I. R. (2003a). Domain organization of the MscS mechanosensitive channel of Escherichia coli. Embo J 22, 36-46.

Miller, S., Edwards, M. D., Ozdemir, C., and Booth, I. R. (2003b). The closed structure of the MscS mechanosensitive channel. Cross-linking of single cysteine mutants. J Biol Chem 278, 32246-32250.

Miyazawa, A., Fujiyoshi, Y., and Unwin, N. (2003). Structure and gating mechanism of the acetylcholine receptor pore. Nature 423, 949-955. 
Moe, P., and Blount, P. (2005). Assessment of potential stimuli for mechanodependent gating of MscL: effects of pressure, tension, and lipid headgroups. Biochemistry 44, 12239-12244.

Nomura, T., Sokabe, M., and Yoshimura, K. (2006). Lipid-protein interaction of the MscS mechanosensitive channel examined by scanning mutagenesis. Biophys J 91, 28742881.

Nomura, T., Sokabe, M., and Yoshimura, K. (2007). Interaction between the cytoplasmic and transmembrane domains of the mechanosensitive channel, MscS. Biophys J.

Okada, K., Moe, P. C., and Blount, P. (2002). Functional design of bacterial mechanosensitive channels. Comparisons and contrasts illuminated by random mutagenesis. J Biol Chem 277, 27682-27688.

Perozo, E. (2006). Gating prokaryotic mechanosensitive channels. Nat Rev Mol Cell Biol 7, 109-119.

Perozo, E., Cortes, D. M., and Cuello, L. G. (1998). Three-dimensional architecture and gating mechanism of a K+ channel studied by EPR spectroscopy. Nat Struct Biol 5, 459469.

Perozo, E., Cortes, D. M., and Cuello, L. G. (1999). Structural rearrangements underlying $\mathrm{K}+$-channel activation gating. Science $285,73-78$.

Perozo, E., Cortes, D. M., Sompornpisut, P., Kloda, A., and Martinac, B. (2002a). Open channel structure of MscL and the gating mechanism of mechanosensitive channels. Nature 418, 942-948.

Perozo, E., Kloda, A., Cortes, D. M., and Martinac, B. (2001). Site-directed spin-labeling analysis of reconstituted Mscl in the closed state. J Gen Physiol 118, 193-206. 
Perozo, E., Kloda, A., Cortes, D. M., and Martinac, B. (2002b). Physical principles underlying the transduction of bilayer deformation forces during mechanosensitive channel gating. Nat Struct Biol 9, 696-703.

Perozo, E., and Rees, D. C. (2003). Structure and mechanism in prokaryotic mechanosensitive channels. Curr Opin Struct Biol 13, 432-442.

Phillips, J. C., Braun, R., Wang, W., Gumbart, J., Tajkhorshid, E., Villa, E., Chipot, C., Skeel, R. D., Kale, L., and Schulten, K. (2005). Scalable molecular dynamics with NAMD. J Comput Chem 26, 1781-1802.

Pivetti, C. D., Yen, M. R., Miller, S., Busch, W., Tseng, Y. H., Booth, I. R., and Saier, M. H., Jr. (2003). Two families of mechanosensitive channel proteins. Microbiol Mol Biol Rev 67, 66-85.

Rasmussen, A., Rasmussen, T., Edwards, M. D., Schauer, D., Schumann, U., Miller, S., and Booth, I. R. (2007). The Role of Tryptophan Residues in the Function and Stability of the Mechanosensitive Channel MscS from Escherichia coli. Biochemistry 46, 1089910908.

Rees, D. C., DeAntonio, L., and Eisenberg, D. (1989). Hydrophobic organization of membrane proteins. Science 245, 510-513.

Schumann, U., Edwards, M. D., Li, C., and Booth, I. R. (2004). The conserved carboxyterminus of the MscS mechanosensitive channel is not essential but increases stability and activity. FEBS Lett 572, 233-237.

Sompornpisut, P., Perozo, E., and Roux, B. (2007). Structural Refinement of Membrane Proteins on the Basis of Experimentally-determined Solvent Accessibility from Spin- 
Label Electron Paramagnetic Resonance Spectroscopy., In 51st Annual Meeting. (Baltimore, Maryland: Biophysical Society).

Sotomayor, M., and Schulten, K. (2004). Molecular dynamics study of gating in the mechanosensitive channel of small conductance MscS. Biophys J 87, 3050-3065.

Sotomayor, M., van der Straaten, T. A., Ravaioli, U., and Schulten, K. (2006).

Electrostatic properties of the mechanosensitive channel of small conductance MscS. Biophys J 90, 3496-3510.

Sotomayor, M., Vasquez, V., Perozo, E., and Schulten, K. (2007). Ion Conduction through MscS as Determined by Electrophysiology and Simulation. Biophys J 92, 886902.

Spronk, S. A., Elmore, D. E., and Dougherty, D. A. (2006). Voltage-dependent hydration and conduction properties of the hydrophobic pore of the mechanosensitive channel of small conductance. Biophys J 90, 3555-3569.

Steinbacher, S., Bass, R., Strop, P., and Rees, D. C. (2007). Structures of the prokaryotic mechanosensitive channels MscL and MscS, Vol 58: Elsevier Inc.).

Strop, P., and Brunger, A. T. (2005). Refractive index-based determination of detergent concentration and its application to the study of membrane proteins. Protein Sci 14, 22072211.

Sukharev, S. (2002). Purification of the small mechanosensitive channel of Escherichia coli $(\mathrm{MscS})$ : the subunit structure, conduction, and gating characteristics in liposomes. Biophys J 83, 290-298.

Sukharev, S., Betanzos, M., Chiang, C. S., and Guy, H. R. (2001a). The gating mechanism of the large mechanosensitive channel MscL. Nature 409, 720-724. 
Sukharev, S., Durell, S. R., and Guy, H. R. (2001b). Structural models of the MscL gating mechanism. Biophys J 81, 917-936.

Takeshi, N., Sokabe, M., and Yoshimura, K. (2006a). Lipid-protein interaction of the MscS mechanosensitive channel examined by scanning mutagenesis. Biophys J. Takeshi, N., Yoshimura, K., and Sokabe, M. (2006b). Voltage dependence of the adaptation in $\mathrm{MscS}$ occurs independent of the charged residues in the transmembrane domain, In Biophysical Society Meeting (Salt Lake City, UT: Biophysical Society). Vasquez, V., Cortes, D. M., Furukawa, H., and Perozo, E. (2007a). An Optimized Purification and Reconstitution Method for the MscS Channel: Strategies for Spectroscopical Analysis. Biochemistry 46, 6766-6773.

Vasquez, V., and Perozo, E. (2004). Voltage dependent gating in MscS, In Biophysical Society Meeting (Baltimore, MD: Biophysical Society), pp. 545A-545A.

Vasquez, V., Sotomayor, M., Cortes, D. M., Roux, B., Schulten, K., and Perozo, E. (2007b). Three Dimensional Architecture of Membrane-Embedded MscS in the Closed Conformation. Journal of Molecular Biology.

Wiener, M. C., and White, S. H. (1992). Structure of a fluid dioleoylphosphatidylcholine bilayer determined by joint refinement of x-ray and neutron diffraction data. III. Complete structure. Biophys J 61, 434-447. 